Fusion Energy Division

\title{
PHYSICS MODELS IN THE TOROIDAL TRANSPORT CODE PROCTR
}

H. C. Howe

DATE PUBLISHED - August 1990

Prepared for the

Office of Fusion Energy

Budget Activity No. AT 05

Prepared by the

OAK RIDGE NATIONAL LABORATORY

Oak Ridge, Tennessee 37831 operated by

MARTIN MARIETTA ENERGY SYSTEMS, INC.

for the

U.S. DEPARTMENT OF ENERGY

under contract DE-AC05-84OR21400 


\section{CONTENTS}

ABSTRACT $\ldots \ldots \ldots \ldots \ldots \ldots \ldots \ldots \ldots \ldots \ldots \ldots \ldots \ldots \ldots$

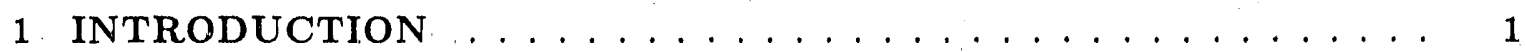

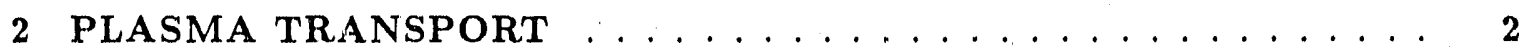

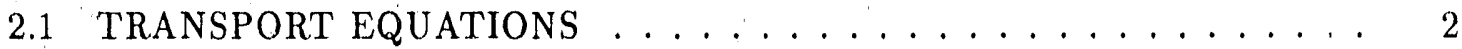

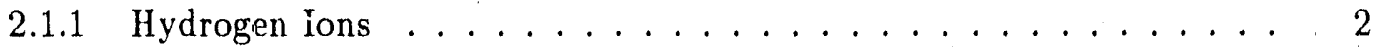

2.1 .2 Helium Ions . . . . . . . . . . . . . . . . . . 2

2.1 .3 Impurity Ions . . . . . . . . . . . . . . . . . 3

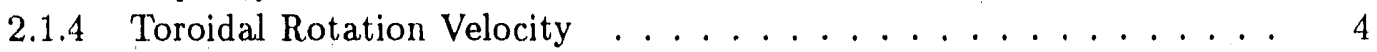

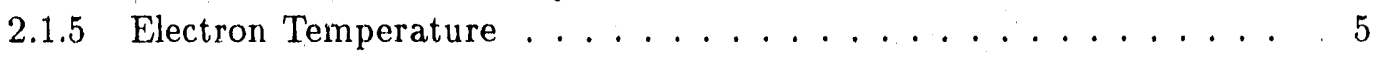

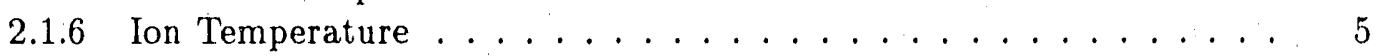

2.1.7 Rotational Transform Profile . . . . . . . . . . . . . 6

2.1 .8 Boundary Conditions . . . . . . . . . . . . . 7

2.1 .9 Initial Conditions . . . . . . . . . . . . . . . . 7

2.2 PLASMA AND TRANSPORT PARAMETERS . . . . . . . . . . 9

2.2 .1 Plasma Parameters . . . . . . . . . . . . . . . 9

2.2.2 Hydrogen and Helium Ion Transport Coefficients . . . . . . . . 10

2.2.3 Impurity Ion Transport Coefficients . . . . . . . . . . . . . 11

2.2 .4 Perpendicular Viscosity . . . . . . . . . . . . . . . . 11

2.2.5 Electron Heat Conduction Coefficient . . . . . . . . . . . . 12

2.2.6. Ion Heat Conduction Coefficient . . . . . . . . . . . . 13

2.2 .7 Helical Ripple Transport . . . . . . . . . . . . . . . . . . . . 14

2.2 .8 Resistivity . . . . . . . . . . . . . . . . . . . . . . . . . . . . . . . . .

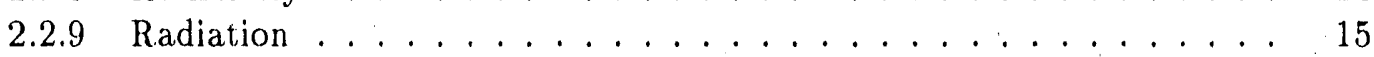

2.2 .10 Radial Potential . . . . . . . . . . . . . . 16

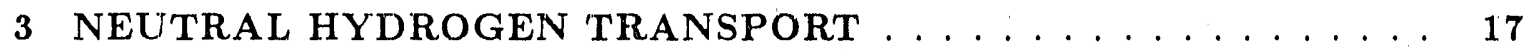

3.1 MULTIGENERATION CHARGE-EXCHANGE TRANSPORT . . . . . . . 17

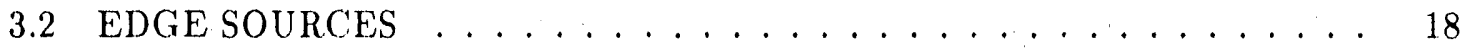

3.2 .1 Cold Wall Source . . . . . . . . . . . . . . . . . . . 18

3.2 .2 Cold Limiter Source . . . . . . . . . . . . . . . . . . . . . . . 20

3.2 .3 Reflected Source . . . . . . . . . . . . . . . . 20

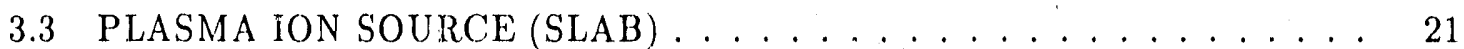

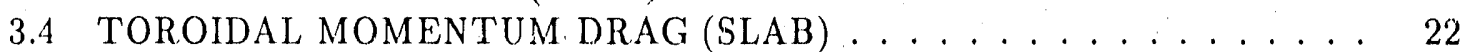

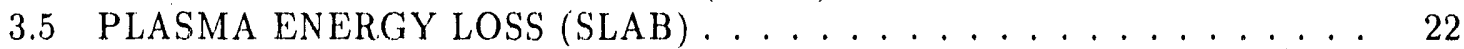

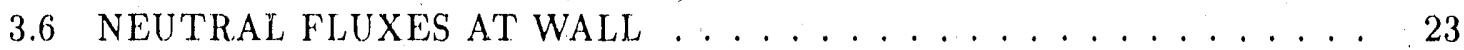

3.6 .1 Particle Fluxes . . . . . . . . . . . . . . . . . . 23

3.6 .2 Energy Fluxes . . . . . . . . . . . . . . . . . . . . 24

3.6.3 Sputtered Impurity Fluxes . . . . . . . . . . . . . . . . . . . . 24

3.6 .4 Sputter Yield Coefficient . . . . . . . . . . . . . . . . 24

3.7 HYDROGEN DEPOSITION PROFILE IN WALI . . . . . . . . . . 24

3.8 NORMALIZED TOROIDAL PARAMETERS AND PROFILES . . . . . 25 


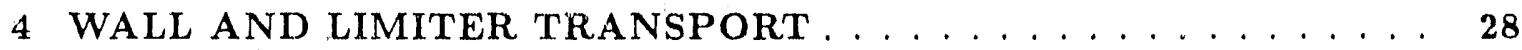

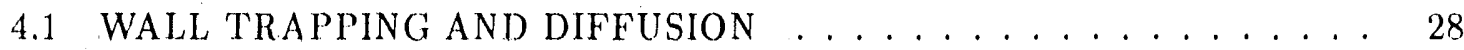

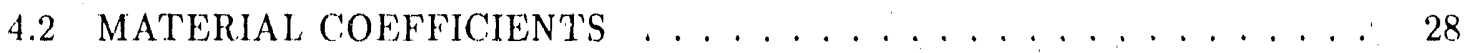

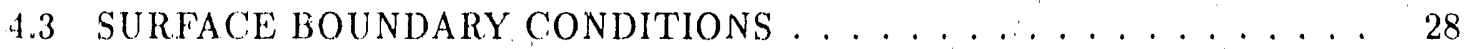

4.4 LIMITER TRANSPORT $\ldots \ldots \ldots \ldots \ldots \ldots$

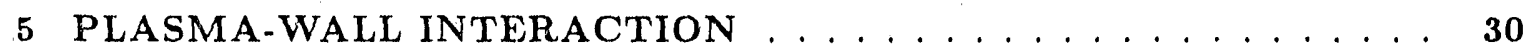

5.1 COUPLED PLASMA AND WALL HYDROGEN SOURCES . . . . . . . 30

5.1.1 Neutral Source Fluxes at Surfaces . . . . . . . . . . . . . . . . 30

5.1.2 Plasma, Wall, and Limiter Particle Sources . . . . . . . . . . . . 31

5.1.3 Plasma Toroidal Momenturn Loss . . . . . . . . . . . . . . . . . . . 32

5.1 .4 Plasma Energy Loss . . . . . . . . . . . . . . . . . . . . . . . 32

5.1 .5 Neutral Dersity . . . . . . . . . . . . . . . . . 32

5.1 .6 Average Recycle and Pumping Coefficient . . . . . . . . . . 33

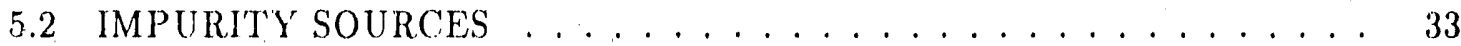

5.2 .1 Wall Charge-Exchange Neutral Sputtering . . . . . . . . . . 33

5.2 .2 Limiter Ion Sputtering . . . . . . . . . . . . . . . . . . . . 34

5.2 .3 Wall and Limiter Recycling . . . . . . . . . . . . . . . . . . . 34

5.2 .4 External Source. . . . . . . . . . . . . . . . . . . 35

5.3 PLASMA SCRAPE-OFF PARALLEL LOSS . . . . . . . . . . . 35

5.3 .1 Parallel Loss Time . . . . . . . . . . . . . . . . . 35

5.3 .2 Energy Loss Factor . . . . . . . . . . . . . . . . 36

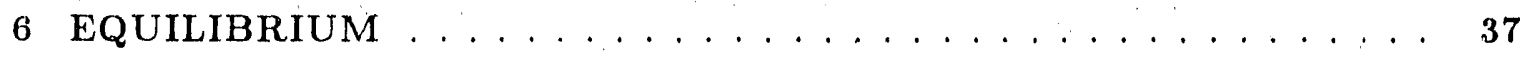

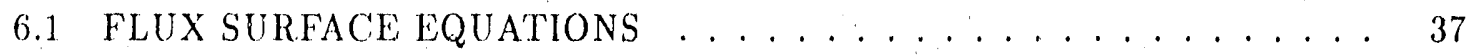

6.1 .1 Axisymmetric Surfaces . . . . . . . . . . . . . . . . 37

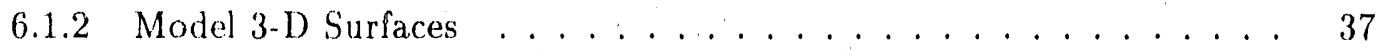

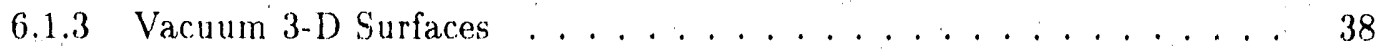

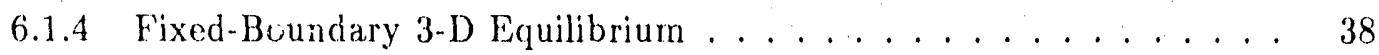

6.1 .5 Scrape-Off Surfaces . . . . . . . . . . . . . . . . . 38

6.1 .6 Equivolume Average Surfaces . . . . . . . . . . . . . . . 39

6.2 MAGNETIC FIELDS AND FLUXES . . . . . . . . . . . . . . . . . . . 40

6.3 PLASMA TRANSPORT METRICS . . . . . . . . . . . . . . . . 40

6.4 MAGNETIC DIFFUSION METRICS . . . . . . . . . . . . 41

6.5 AXISYMMETRIC, SHIFTED, ELLIPTICAL SURFACES . . . . . . . . 41

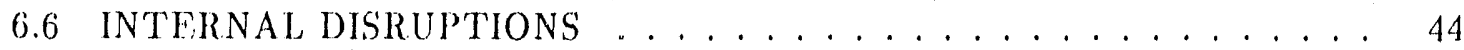

6.6.1 Transport Coefficient Enhancement. . . . . . . . . . . . 44

6.6 .2 Sawtooth Model . . . . . . . . . . . . . . . . 45

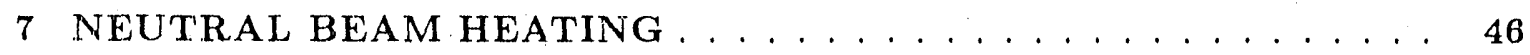

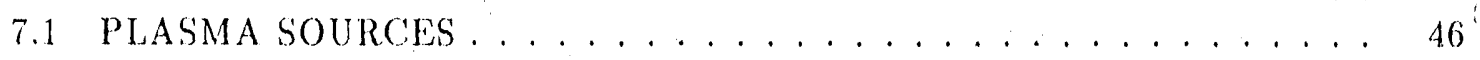

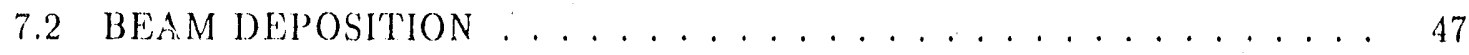

7.2 .1 Diffuse-Beam Integration . . . . . . . . . . . . . . 47

7.2 .2 Pencil-Beam Deposition . . . . . . . . . . . . . . 48

7.3 FAST-ION THERMALIZATION . . . . . . . . . . . 49

7.3.1 Moments of Fokker-Planck Equation . . . . . . . . . . . . 49 
7.3.2 Complete Fokker-Planck Equation . . . . . . . . . . . . 51

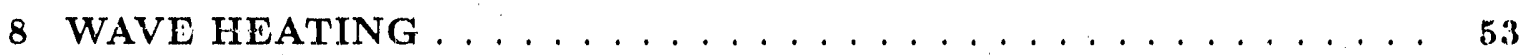

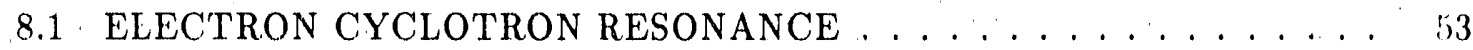

8.1.1 Resonance Layer Model . . . . . . . . . . . . . . . . . . . . . . . . . . . . . . . 53

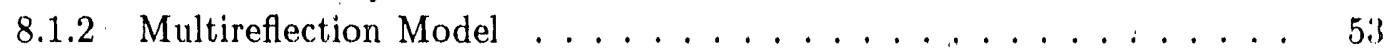

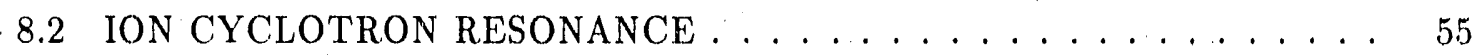

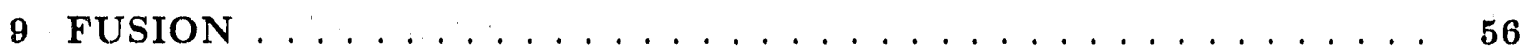

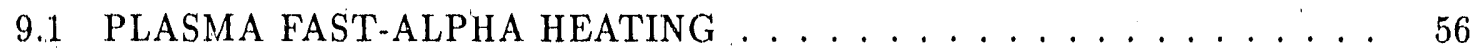

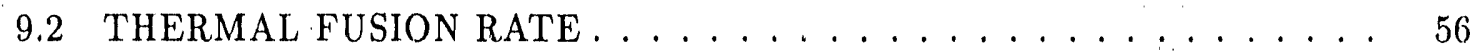

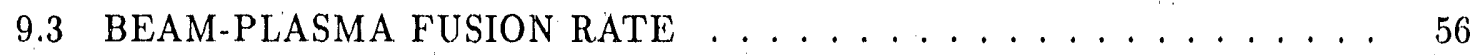

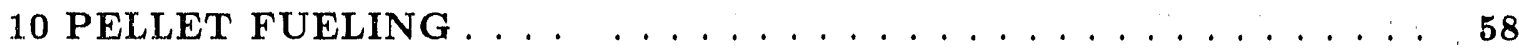

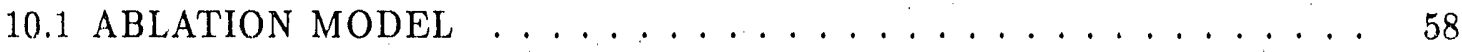

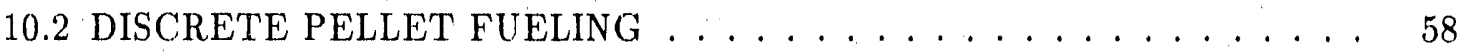

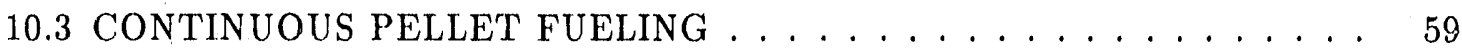

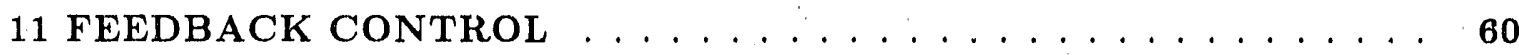

11.1 PLASMA DENSITY CONTROL BY GAS FEED RATE . . . . . . . . . 60

11.2 LINE DENSITY CONTROL BY PARTICLE DIFFUSIVITY . . . . . . . . 60

11.3 LINE DENSITY CONTROL BY LIMITER RECYCLING . . . . . . . . . . 61

11.4 FUSION POWER CONTROL BY VARIABLE DENSITY . . . . . . . . 61

11.5 FUSION POWER CONTROL BY TOROIDAL FIELD RIPPLE . . . . . . 61

11.6 ION TEMPERATURE CONTROL BY ION CONDUCTIVITY . . . . . . 62

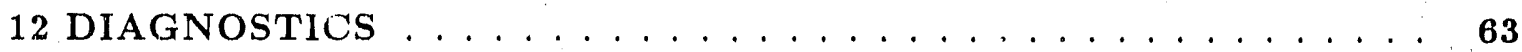

12.1 DIAGNOSTIC VALUES FOR EXPERIMENTAL COMPARISON . . . . 63

12.1 .1 Line-Average Density . . . . . . . . . . . . . 63

12.1 .2 Loop Voltage . . . . . . . . . . . . . . . . . . . . 63

12.1 .3 Beta . . . . . . . . . . . . . . . . . 63

12.1.4 Energy Confinement Time . . . . . . . . . . . . . . . . . . 64

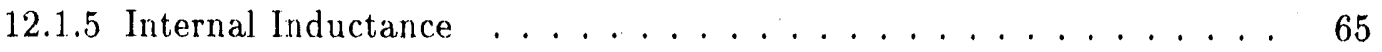

12.1.6 Safety Factor . . . . . . . . . . . . . . . . . . . 65

12.1.7 Neutron Rate . . . . . . . . . . . . . . . . 65

12.1.8 Radiated Power . . . . . . . . . . . . . . . . 65

12.1.9 Charge-Exchange Ion Temperature . . . . . . . . . . 65

12.1.10Spectroscopic Lines . . . . . . . . . . . . . . . . . . . 66

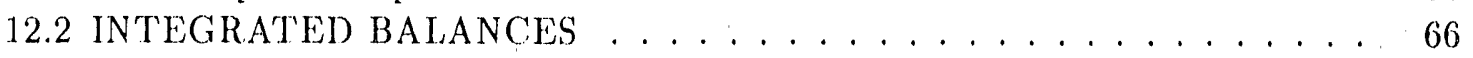

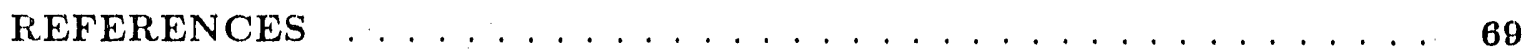

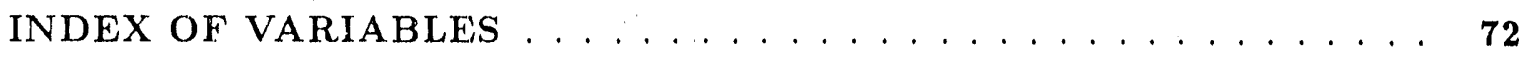

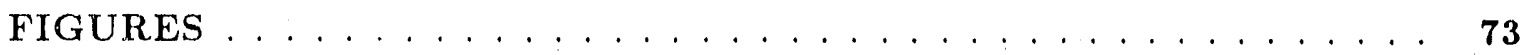




\begin{abstract}
The physics models that are contained in the toroidal transport code PROCTR are described in detail. Time- and space-dependent models are included for the plasma hydrogrnicion, helium, and impurity densities, the electron and ion temperatures, the toroidal rotation velocity, and the toroidal current profile. Time- and depth-dependent models for the trapped and mobile hydrogenic particle concentrations in the wall and a time-dependent point model for the number of particles in the limiter are also included. Time-independent models for neutral particle transport, neutral beam deposition and thermalization, fusion heating, impurity radiation, pellet injection, and the radial electric potential are included and recalculated periodically as the time-dependent models evolve. The plasma solution is obtained either in simple flux coordinates, where the radial shift of each elliptical, toroidal flux surface is included to maintain an approximate pressu re equilibriurn, or in general threedimensional torsatron coordinates represented by a series of helical harmonics. The detailed coupling of the plasma, scrape-off layer, limiter, and wall models through the neutral transport model makes PROCTR especially suited for modeling of recycling and particle control in toroidal plasmas. The model may also be used in a steady-state profile analysis mode for studying energy and particle balances starting with measured plasma profiles.
\end{abstract}




\section{INTRODUCTION}

This report describes the physics models that are contained in the toroidal transport code PROCTR. For each model, the equations are given in full detail along with a terse physical description. There is no description of the programming aspects of the transport code; that is, this is a physics description and not a code description. However, the structure of the equations parallels the structure of the code in many places, making this report useful for understanding the coding.

The complete plasma model is made up of time- and space-dependent models for plasma. and wall parameters. In addition, time-independent models are included for sources and sinks. Time-dependent spatial profiles are evolved for the plasma ion densities (one or two species), thermal-alpha density (for deuterium-tritium burning plasmas), electron and ion temperatures, toroidal rotation velocity, impurity ion densities (one or two species), and the toroidal current density. For the chamber wall, time-dependent depth profiles are evolved for particle concentrations (both mobile and trapped) corresponding to the plasma ion species.

Time-independent models are included for neutral hydrogen transport, neutral beam deposition and thermalization, fusion heating, impurity radiation, the radial electric potential, pellet ablation, and the plasma equilibrium. The plasma solution may be obtained in simplified flux surface coordinates, where each flux surface is assumed to be an elongated torus shifted radially with respect to adjacent surfaces to maintain an approximate pressure equilibrium. Alternatively, nonaxisymmetric three-dimensional (3-D) flux surfaces may be represented by a set of helical harmonics.

A time-dependent point model is included for the number of particles in the limiter. The models for plasma, limiter, and wall particle transport, along with the scrape-off layer and neutral transport models, compose a complete and detailed plasma recycle model.

The models are extensively cross-referenced. Italic numbers appearing in parentheses refer to sections within the report. For example, the model for the plasma electron density $n_{e}$ (2.2.1) is given in Sect. 2.2.1. In addition, the index gives section references for all variables that are used in more than one section of the report, except for variables used in the neutral transport model description in Sect. 3.

The bibliography contains only the references used in constructing the models in the transport code. Thus, it is not a complete reference list for toroidal plasma transport modeling and does not contain many interesting papers that report modeling results without detailed model descriptions. The papers cited are of interest primarily for their complete physical descriptions of the models in the code.

PROCTR has been used extensively to model actual tokamak shots by using shot data where appropriate for boundary conditions and by attempting to predict diagnostic measurements for the same shot as a test of the physics models in the code. Definitions of the plasma parameters calculated in the code for comparison with diagnostic measurements are given in Sect. 12. 


\section{PLASMA TRANSPORT}

\subsection{TRANSPORT EQUATIONS}

The plasma transport equations are flux surface averages of the general equations as described, for example, by Braginskii [1]. A review of plasma transport by Blum and Le Foll [2] describes the averaging method.

\subsubsection{Hydrogen Ions}

The density of hydrogenic plasma species $j, n_{H j}$, is governed by

$$
\begin{aligned}
\frac{\partial n_{H j}}{\partial t} & =S_{H j}(\rho)-S_{\text {fus }}(\rho)-\frac{1}{V_{\rho}^{\prime}} \frac{\partial}{\partial \rho}\left(V_{\rho}^{\prime} \Gamma_{H j}\right)+S_{\mathrm{inj}}(\rho)-\frac{n_{H j}}{\tau_{\| H j}} \\
\Gamma_{H j} & =-\left\langle(\nabla \rho)^{2}\right\rangle_{\psi} D_{H j} \frac{\partial n_{H j}}{\partial \rho}+\langle|\nabla \rho|\rangle_{\psi} n_{H j} v_{\text {pnch }}
\end{aligned}
$$

W. nere $S_{H j}$ is the neutral ionization source term (5.1.2), $S_{\text {fus }}$ is the loss rate of plasma fuel due to thermal fusion (9.2), $S_{\mathrm{inj}}$ is the fueling rate from thermalized fast ions (7.1), and the $\tau_{\|}$term accounts for parallel loss of plasma to the limiter or divertor in the scrape-off layer (5.3.1). In the radial particle flux $\left(\Gamma_{H j}\right), D_{H j}$ is the diffusivity (2.2.2) and $v_{\text {pnch }}$ is the radial convective velocity (2.2.2).

If the hydrogenic-ion equations are not solved, the hydrogenic-ion density profiles $n_{H j}(\rho)$ may be either specified arbitrarily (2.1.9) or calculated from a specified $n_{e}(\rho)$ (for example, the measured laser profile) to satisfy charge neutrality

$$
\begin{aligned}
& n_{H 1}=\frac{1}{1+f_{n}}\left(n_{e}-\sum_{j} n_{I j} Z_{j}-n_{b}\right) \\
& n_{H 2}=f_{n} n_{H 1}
\end{aligned}
$$

where the ion density ratio $f_{n}$ is specified arbitrarily. The hydrogenic-ion flux $\Gamma_{H j}$ is calculated, for constant ion density, by assuming that the ion flux across flux surface $\rho$ balances the total rate of plasma creation within the volume contained by the surface. This is equivalent to setting $\Gamma_{H j}$ so that the ion density equation is $\partial n_{H j} / \partial t=0$. Thus, for constant ion density,

$$
V_{\rho}^{\prime}(\rho) \Gamma_{H j}(\rho)=\int_{0}^{\rho}\left(S_{H j}\left(\rho^{\prime}\right)+S_{\mathrm{inj}}\left(\rho^{\prime}\right)-\frac{n_{H, j}\left(\rho^{\prime}\right)}{\tau_{\| H j}\left(\rho^{\prime}\right)}\right) V_{\rho}^{\prime}\left(\rho^{\prime}\right) d \rho^{\prime}
$$

where the source terms are defined above.

\subsubsection{Helium Ions}

The equation for ionized helium is only solved when modeling a burning plasma; that is, this is a helium ash equation. The density of the helium component of the plasma $n_{\mathrm{He}}$ is governed by

$$
\begin{aligned}
\frac{\partial n_{\mathrm{He}}}{\partial t} & =S_{\mathrm{fus}}(\rho)-\frac{1}{V_{\rho}^{\prime}} \frac{\partial}{\partial \rho}\left(V_{\rho}^{\prime} \Gamma_{\mathrm{He}}\right)-\frac{n_{\mathrm{He}}}{\tau_{\| \mathrm{He}}} \\
\Gamma_{\mathrm{He}} & =-\left\langle(\nabla \rho)^{2}\right\rangle_{\psi^{\prime}} D_{\mathrm{He}} \frac{\partial n_{\mathrm{He}}}{\partial \rho}
\end{aligned}
$$


where $S_{\text {fus }}$ is the source due to fusion (9.2). The $\tau_{\|}$term accounts for parallel loss to the limiter or divertor in the scrape-off layer, including the reduction of the loss rate due to helium recycling (5.3.1). In the radial particle flux $\Gamma_{\mathrm{He}}, D_{\mathrm{He}}$ is the diffusivity (2.2.2).

\subsubsection{Impurity Ions}

The density of impurity species $j, n_{I j}$, may either be specified al bitrarily or evolve radially in time. The temporal evolution may be governed by the averige-ion model, which treats each impurity species with a single equation for the total impurity density. Alternatively, the coupled set of equations for the density of each charge state of an impurity may be solved.

Arbitrary Density If the impurity density equations are not solved, the impurity densities may be either specified arbitrarily (2.1.9) or defined to give an arbitrary value of the plasma effective $Z\left(Z_{\text {eff }}^{s}\right)$. The impurity densities that give $Z_{\text {eff }}(\rho)=Z_{\text {eff }}^{s}(\rho)$ are

$$
\begin{aligned}
& n_{I 1}=\frac{\left(Z_{\mathrm{eff}}^{s}-1\right)\left(n_{e}-n_{b}\right)}{Z_{1}\left(Z_{1}-1\right)+f_{z} Z_{2}\left(Z_{2}-1\right)} \\
& n_{I 2}=f_{z} n_{I 1}
\end{aligned}
$$

where the density ratio $f_{z}$ is specified arbitrarily. The average charge state $Z_{j}$ is given by the tables of Post et al. [3].

Average-Ion Model The density of impurity species $j, n_{I j}$, is governed by

$$
\begin{aligned}
\frac{\partial n_{I j}}{\partial t}= & S_{I j}(\rho)-\frac{1}{V_{\rho}^{\prime}} \frac{\partial}{\partial \rho}\left(V_{\rho}^{\prime} \Gamma_{I j}\right)-\frac{n_{I j}}{\tau_{|| I j}} \\
\Gamma_{I j}= & \left\langle(\nabla \rho)^{2}\right\rangle_{\psi} n_{I j} D_{H} \frac{\left\langle Z_{j}^{2}\right\rangle}{Z_{j}}\left[K_{j}\left(\frac{1}{n_{H}} \frac{\partial n_{H}}{\partial \rho}-\frac{1}{Z_{j} n_{I j}} \frac{\partial n_{I j}}{\partial \rho}\right)+\frac{H_{j}}{T_{i}} \frac{\partial T_{i}}{\partial \rho}\right] \\
& \quad-\left\langle(\nabla \rho)^{2}\right\rangle_{\psi} D_{A I} \frac{\partial n_{I j}}{\partial \rho}+\langle\mid \nabla \rho\rangle_{\psi} n_{I j} v_{A I} \\
n_{H}= & \sum_{j} n_{H j} \\
n_{l}= & \sum_{j} n_{I j}
\end{aligned}
$$

where $S_{I j}$ is the impurity source arising from ionization of wall- and limiter-evolved neutral impurities (5.2) and the $\tau_{\|}$term accounts for parallel loss of impurities to the limiter or divertor in the scrape-off layer (5.3.1). The radial impurity flux $\Gamma_{l, j}$ includes neoclassical fluxes driven by the total hydrogenic-ion density $\left(n_{H}\right)$ gradient, the impurity density gradient, and the ion temperature gradient. The radial flux also includes anomalous spreading with diffusivity $D_{A I}$ (2.2.3) and anomalous convection with velocity vAI (2.2.3). The neoclassical transport coefficients $\left(D_{H}, K_{j}\right.$, and $H_{j}$ ) are given below (2.2.3). The average charge state $Z_{j}$ and $\left\langle Z_{j}^{2}\right\rangle$ are the coronal values given by the tables of Post et al. [3]. 
Multi-Charge-State Model The density of charge state $q$ of impurity species $j, n_{I j}^{q}$, is governed by

$$
\begin{aligned}
\frac{\partial n_{I j}^{q}}{\partial t}= & -\frac{1}{V_{\rho}^{\prime}} \frac{\partial}{\partial \rho}\left(V_{\rho}^{\prime} \Gamma_{I j}^{q}\right)-\frac{n_{I j}^{q}}{\tau_{\| I j}} \\
& +n_{e} n_{I_{j}}^{q-1}\langle\sigma v\rangle_{i}^{q-1}+n_{e} n_{I j}^{q+1}\langle\sigma v\rangle_{r}^{q}-n_{e} n_{I j}^{q}\langle\sigma v\rangle_{i}^{q}-n_{e} n_{I j}^{q}\langle\sigma v\rangle_{r}^{q-1} \\
\Gamma_{I j}^{q}= & -\left\langle(\nabla \rho)^{2}\right\rangle_{\psi} D_{A I} \frac{\partial n_{I j}^{q}}{\partial \rho}+\langle\mid \nabla \rho\rangle_{\psi} n_{I j}^{q} v_{A I} \\
n_{I j}^{q=0}:= & \frac{S_{I j}}{n_{e}\langle\sigma v\rangle_{i}^{q=0}} \\
n_{I j}= & \sum_{q=1}^{Z} n_{I j}^{q} \\
Z_{j}= & \frac{1}{n_{I j}} \sum_{q=1}^{Z} q n_{I j}^{q} \\
\left\langle Z_{j}^{2}\right\rangle= & \frac{1}{n_{I j}} \sum_{q=1}^{Z} q^{2} n_{I j}^{q}
\end{aligned}
$$

where $q=1 \rightarrow Z$ for charge states of an impurity with total nuclear charge $Z$ and $q=0$ for the neutral impurity density. $\langle\sigma v\rangle_{i}^{q}$ is the rate of ionization of impurities in charge state $q$ into charge state $q+1 .\langle\sigma v\rangle_{r}^{q}$ is the rate of recombination of impurities in charge state $q+1$ into charge state $q . S_{I j}$ is the impurity source arising from ionization of wall- and limiterevolved neutral impurities (5.2) and the $\tau_{\|}$term accounts for parallel loss of impurities to the limiter or divertor in the scrape-off layer (5.3.1). The radial flux includes anomalous spreading with diffusivity $D_{A I}$ (2.2.3) and anomalous convection with velocity $v_{A I}$ (2.2.3).

\subsubsection{Toroidal Rotation Velocity}

The toroidal momentum density of the plasma $p_{\phi}$ is governed by

$$
\begin{aligned}
\frac{\partial p_{\phi}}{\partial t} & =\dot{p}_{\phi}^{T}-\frac{1}{V_{\rho}^{\prime}} \frac{\partial}{\partial \rho}\left(V_{\rho}^{\prime} p_{\phi} \frac{\Upsilon_{T}}{n_{H}}\right)+\frac{1}{V_{\rho}^{\prime}} \frac{\partial}{\partial \rho}\left(V_{\rho}^{\prime}\left\langle(\nabla \rho)^{2}\right\rangle_{\psi} \eta_{\perp} \frac{\partial}{\partial \rho} \frac{p_{\phi}}{\rho_{m}}\right)+\dot{p}_{\mathrm{cx}}-\dot{p}_{\|} \\
\rho_{m} & =\sum_{j} m_{H j} n_{H j}+\sum_{j} m_{I j} n_{I j} \\
\Gamma_{T} & =\sum_{j} \Gamma_{H j} \\
v_{\phi} & =\frac{p_{\phi}}{\rho_{m}} \\
\dot{p}_{\|} & =p_{\phi}\left(\frac{\left|v_{\phi}\right|}{2 \pi R_{0}}+\sum_{j} \frac{1}{\tau_{\| H j}}\right)
\end{aligned}
$$

where $\dot{p}_{\phi}^{T}$ is the driving toroidal momentum source due to injected fast ions $(7.1), \dot{p}_{\mathrm{cx}}$ is the toroidal drag exerted by charge-exchange loss of neutrals (5.1.3), and $\dot{\psi}_{\|}$is the drag exerted 
by the limiter. $v_{\phi}$ is the toroidal rotation velocity, $\rho_{m}$ is the total plasma mass density, $\Gamma_{T}$ is the total radial ion flux, and $\eta_{\perp}$ is the perpendicular ion viscosity (2.2.4). The two radial transport terms represent momentum transport due to radial mass convection and viscosity, respectively.

\subsubsection{Electron Temperature}

The electron temperature $T_{e}$ is governed by

$$
\begin{aligned}
\frac{3}{2} n_{e} \frac{\partial T_{e}}{\partial t}= & q_{\mathrm{inj}}^{e}(\rho)-\frac{1}{V_{\rho}^{\prime}} \frac{\partial}{\partial \rho}\left(V_{\rho}^{\prime} q_{e}\right)-3 \frac{m_{e}}{m_{p}} \frac{n_{e}}{\tau_{e}}[Z]\left(T_{e}-T_{i}\right) \\
& +q_{\mathrm{rad}}(\rho)+q_{\mathrm{ion}}(\rho)+q_{\mathrm{oh}}(\rho)+q_{\mathrm{ec}}(\rho)+q_{\mathrm{cc}}^{e}(\rho)+q_{\mathrm{fus}}^{e}(\rho) \\
& -2 \gamma T_{e} \dot{n}_{\|}-\frac{3}{2} T_{e} \frac{\partial n_{e}}{\partial t} \\
q_{e}= & -\left\langle(\nabla \rho)^{2}\right\rangle_{\psi} n_{e} \chi_{e} \frac{\partial T_{e}}{\partial \rho}+q_{e}^{n a}+\frac{3}{2} T_{e} \Gamma_{e} \\
\Gamma_{e}= & \sum_{j} \Gamma_{H j}+Z_{\mathrm{He}} \Gamma_{\mathrm{He}}+\sum_{j} Z_{j} \Gamma_{I j} \\
\dot{n}_{\|}= & \sum_{j} \frac{n_{H j}}{\tau_{\| H j}}+\frac{n_{\mathrm{He}}}{\tau_{\| \mathrm{He}}}
\end{aligned}
$$

where the first three terms represent neutral beam heating (7.1), radial transport, and electron-ion rethermalization, respectively. Radial transport $\left(q_{e}\right)$ includes heat conduction with the coefficient $\chi_{e}$ (2.2.5), conduction $q_{c}^{n a}$ due to helical ripple (2.2.7) and convection. Ambipolarity determines the radial electron flux $\Gamma_{e}$, which is the sum of the hydrogenic-ion flux $\Gamma_{H j}$ (2.1.1), the helium-ion flux $\Gamma_{\mathrm{He}}$ (2.1.2), and the impurity-ion flux $\Gamma_{I j}$ (2.1.3). The electron collision time is $\tau_{e}$ (2.2.1). Impurity and anomalous radiation losses are given by $q_{\mathrm{rad}}(2.2 .9), q_{\mathrm{ion}}$ is the ionization and radiation loss from neutrals $(5.1 .4), q_{\mathrm{oh}}$ is the ohmic heating (2.1.7), and $q_{\text {fus }}^{e}$ is heating due to fusion alpha particles (9.1). Electron cyclotron resonance heating is represented by $q_{\mathrm{ec}}(8.1)$ and electron heating by ion cyclotron resonance heating is given by $q_{\mathrm{ic}}^{e}(8.2)$. The $\dot{n}_{\|}$term accounts for parallel energy loss to the limiter or divertor in the scrape-off (5.3.1), where $\gamma$ is the sheath enhancement factor (5.3.2).

\subsubsection{Ion Temperature}

The temperature of plasma ions and impurities $T_{i}$ is governed by

$$
\begin{aligned}
\frac{3}{2} n_{T} \frac{\partial T_{i}}{\partial t}= & 3 \frac{m_{e}}{m_{p}} \frac{n_{e}}{\tau_{e}}[Z]\left(T_{e}-T_{i}\right)-\frac{1}{V_{\rho}^{\prime}} \frac{\partial}{\partial \rho}\left(V_{\rho}^{\prime} q_{i}\right) \\
& \quad-q_{\mathrm{cx}}(\rho)+q_{\mathrm{inj}}^{i}(\rho)+q_{\mathrm{ic}}^{i}(\rho)+q_{\text {fus }}^{i}(\rho)-2 T_{i}^{\prime} n_{\|}-\frac{3}{2} T_{i} \frac{\partial n_{T}}{\partial t} \\
q_{i}= & \sum_{j}\left(-\left\langle(\nabla \rho)^{2}\right\rangle_{\psi} n_{H j} \chi_{H j} \frac{\partial T_{i}}{\partial \rho}+q_{H j}^{n a}+\frac{3}{2} T_{i} \Gamma_{H j}\right) \\
n_{T}= & n_{H}+n_{\text {He }}+n_{I}
\end{aligned}
$$

where the first two terms are electron-jon rethermalization and radial transport, respectively. Radial transport $\left(q_{i}\right)$ includes heat conduction with the coefficient $x_{H}$ (2.2.6), conduction 
$q_{H j}^{n a}$ due to helical ripple (2.2.7) and convection due to ion particle flux $\Gamma_{H j}$ (2.1.1). Energy loss due to neutral charge exchange is given by $q_{\mathrm{cx}}(5.1 .4), q_{\mathrm{inj}}^{i}$ is ion neutral beam heating (7.1), $q_{\mathrm{ic}}^{i}$ is ion-cyclotron resonance heating (8.2), $q_{\text {fus }}^{i}$ is ion heating due to fusion alpha particles (9.1), and parallel heat loss to the limiter or divertor is given by the term containing $\dot{n}_{\|}(2.1 .5) . n_{T}$ is the total plasina ion density.

\subsubsection{Rotational Transform Profile}

The resistive diffusion of the total current $I(\rho, t)$ contained within each fux surface $\rho$ is governed by

$$
\frac{\partial I(\rho)}{\partial t}=\frac{1}{\mu_{0}} c_{\theta} r_{\rho} \frac{\partial}{\partial r_{\rho}}\left(\frac{\eta_{f}}{c_{\phi} r_{\rho}} \frac{\partial I(\rho)}{\partial r_{\rho}}\right)-\frac{1}{\mu_{0}} 2 \pi c_{\theta} r_{\rho} \frac{\partial}{\partial r_{\rho}}\left(\eta_{f} J_{b}(\rho)\right)
$$

where $\eta_{f}$ is the parallel resistivity $\eta_{\|}$(2.2.8) modified inside the $q=1$ surface to simulate the effect of sawteeth on the current profile $(6.6 .1) . J_{b}$ is the shielded current perturbation driven by injected fast ions (7.1). The geometric constarts $c_{\phi}$ and $c_{\theta}(6.4)$ are flux surface averages of the metric coefficients $g_{i j}$ for the axisymmetric surfaces.

Instead of following the evolution of the toroidal current on the resistive time scale, the instantaneous steady-state current profile may be calculated. This current profile will evolve with time-dependent changes in the parallel resistivity profile $\eta_{f}$, the total plasma current $I_{0}(t)$, and the beam-driven current density $J_{b}$. The steady-state current profile is given by

$$
\begin{aligned}
I(\rho) & =I_{p}(\rho)+I_{b}(\rho) \\
I_{b}(\rho) & =\int_{0}^{r_{\rho}} 2 \pi r_{\rho}^{\prime} c_{\phi} J_{b} d r_{\rho}^{\prime} \\
I_{p}(\rho) & =\left(I_{0}(t)-I_{b}(\rho=1)\right)\left(\int_{0}^{r_{\rho}} \frac{1}{\eta_{f}} r_{\rho}^{\prime} c_{\phi} d r_{\rho}^{\prime}\right) /\left(\int_{0}^{r_{a}} \frac{1}{\eta_{f}} r_{\rho}^{\prime} c_{\phi} d r_{\rho}^{\prime}\right)
\end{aligned}
$$

where $I_{p}$ is the current carried by the plasma and $I_{b}$ is the current carried by the fast beam ions including shielding by the plasma electrons.

The average poloidal magnetic field due to plasma current $B_{\theta}^{I}$, the toroidal current density $J_{z}$, and the toroidal electric field $E_{z}$ are obtained from the total current profile $I(\rho, t)$ by

$$
\begin{aligned}
B_{\theta}^{I} & =\frac{\mu_{0} I(\rho)}{2 \pi c_{\theta} r_{\rho}} \\
J_{z} & =\frac{1}{\mu_{0}} \frac{1}{c_{\phi} r_{\rho}} \frac{\partial}{\partial r_{\rho}}\left(c_{\theta} r_{\rho} B_{\theta}^{I}\right) \\
E_{z} & =\eta_{\eta}\left(J_{z}-J_{b}\right)
\end{aligned}
$$

The equivalent toroidal current density used in the ohmic heating term $\left(q_{\mathrm{oh}}\right)$ of the electron temperature equation (2.1.5) is

$$
\begin{aligned}
& J_{\mathrm{oh}}=\frac{1}{\mu_{0}} \frac{(2 \pi)^{2} R_{0}}{V_{\rho}^{\prime}} \frac{\partial}{\partial \rho}\left(c_{\theta} r_{\rho} B_{\theta}^{I}\right) \\
& q_{\mathrm{oh}}=E_{z} J_{\mathrm{oh}}
\end{aligned}
$$


The average vacuum poloidal magnetic field $B_{\theta}^{\nu}$ is given in terms of the vacuum rotational transform $t_{v}$ by

$$
B_{\theta}^{v}=t_{v} \frac{c_{\phi} r_{\rho} B_{T}}{R_{0}}
$$

and the total average poloidal magnetic field is $B_{\theta}=B_{\theta}^{v}+B_{\theta}^{I}$. The total rotational transform $t$ is defined below (2.2.1).

\subsubsection{Boundary Conditions}

At the plasma center $(\rho=0)$, a zero-slope boundary condition is used for $n_{H j}, n_{\mathrm{He}}$, $n_{I j}, v_{\phi}, T_{e}$, and $T_{i}$. Thus, if the variable $X$ is any of these plasma parameters, the central boundary condition is

$$
\frac{\partial X(\rho, t)}{\partial \rho}=0 \text { for } \rho=0
$$

For whe toroidal current equation (2.1.7), $I(\rho=0, t)=0$.

At the plasma edge $(\rho=a)$, arbitrary, constant values are assigned to $n_{H j}, n_{I j}, v_{\phi}, T_{e}$, and $T_{i}$. Thus, if $X$ represents any of these variables,

$$
X(\rho, t)=X_{0} \text { for } \rho=a
$$

Helium ions are assumed to be perfectly reflected from the plasma outer boundary. These ions may leave the system only by limiter or divertor pumping (5.3.1). Thus, the helium ion edge boundary condition is

$$
\frac{\partial n_{\mathrm{He}}(\rho, t)}{\partial \rho}=0 \quad \text { at } \quad \rho=a
$$

The toroidal current at the edge may be any specified time-dependent value $I(\rho=a, t)=$ $I_{0}(t)$, where $I_{0}(t)$ is the total toroidal current. Alternatively, the loop voltage may be zero, in which case the toroidal current boundary condition is

$$
\frac{\partial I(\rho, t)}{\partial \rho}=0 \text { at } \rho=a
$$

\subsubsection{Initial Conditions}

At the initial time $t=t_{0}$, arbitrary magnitudes and profile shapes are specified for the electron temperature $T_{e}\left(\rho, t_{0}\right)(2.1 .5)$, the ion temperature $T_{i}\left(\rho, t_{0}\right)(2.1 .6)$, the impurity densities $n_{I j}\left(\rho, t_{0}\right)$ (2.1.3), the helium-ion density $n_{\mathrm{He}}\left(\rho, t_{0}\right)(2.1 .2)$, and the wall concentrations of trapped hydrogen $c_{T j}\left(y, t_{0}\right)(4.1)$. The initial toroidal momentum density $p_{\phi}\left(\rho, t_{0}\right)=0(2.1 .4)$.

The initial profile of a plasma parameter $X\left(\rho, t_{0}\right)$ (which may be $T_{e}, T_{i}, n_{e}, n_{H j}, n_{I j}$, or $\left.n_{\text {He }}\right)$ is given by one of two forms. The exponential form is

$$
X^{\prime}(\rho)= \begin{cases}\left(X_{b}-X_{0}\right)\left[1-\left(\rho / \rho_{0}\right)^{c}\right]^{\beta}+X_{0}, & \rho<\rho_{0} \\ X_{0}, & \rho>\rho_{0}\end{cases}
$$


where the profile is specified by the parameter set $\left(X_{b}, X_{o}, \alpha, \beta, \rho_{0}\right)$. The Gaussian form is

$$
\begin{aligned}
X(\rho) & =\frac{1}{1-f_{0}}\left[\left(X_{b}-X_{0}\right) e^{-(\rho / \Delta)^{\alpha}}+X_{o}-X_{b} f_{0}\right] \\
f_{0} & =\exp \left[-\left(\rho_{0} / \Delta\right)^{\alpha}\right]
\end{aligned}
$$

where the profile is specified by the parameter set $\left(X_{b}, X_{o}, \Delta, \alpha, \rho_{0}\right)$. For both profiles, $X_{b}$ is the value at $\rho=0$ and $X_{o}$ is the value at $\rho=\rho_{0}$.

The initial profile shape of the mobile hydrogen concentration of species $j$ in the wall $c_{j}\left(y, t_{0}\right)(4.1)$ is assumed to be given by the wall deposition profile $h\left(y, E_{b}\right)$ (3.7) for $E_{b} \equiv$ $10 \mathrm{eV}$. Thus,

$$
c_{j}\left(y, t_{0}\right)=\frac{N_{w j}}{A_{w}} \frac{h\left(y, E_{b}\right)}{\int_{0}^{y_{\max }} h\left(y, E_{b}\right) d y}
$$

where $A_{u}$ is the equivalent wall area $(5,1)$ and $N_{w j}$ is the initial total number of particles in the wall.

For a simulation that does not use actual data from a plasma shot, arbitrary magnitudes and profile shapes are assumed for the hydrogenic-ion densities $n_{H j}\left(\rho, t_{0}\right)$ (2.1.1). For each hydrcgenic species $H j$, the total number of particles in the wall initially, $N_{w j}$, is assumed to be equal to the initial total number of ions in the plasma. Thus, initialiy,

$$
N_{w j}=\int_{0}^{a} n_{H j}\left(\rho, t_{0}\right) V_{\rho}^{\prime} d \rho
$$

The initial number in the limiter $N_{L j}\left(t_{0}\right)=0$ (4.4).

When a plasma shot is simulated, the measured gas feed rate is used to calculate the number of particles-equivalent in the prefill, $N_{\mathrm{pf}}$. This total number is arbitrarily divided between the initial numbers in the plasma, wall, and limiter. The fraction of $N_{\mathrm{pf}}$ in the plasma is $f_{\mathrm{pl}}$, the fraction in the wall is $f_{\mathrm{wl}} f_{\mathrm{cx}}^{w}$, and the fraction in the limiter is $f_{\mathrm{wl}} f_{\mathrm{cx}}^{L}$. Here, $f_{\mathrm{wl}}=1-f_{\mathrm{pl}}$ is the fraction of $N_{\mathrm{pf}}$ initially in the wall and limiter, and $f_{\mathrm{cx}}^{w, L}$ are the fractions of the charge-exchange efflux from the plasma incident on the wall and limiter, respectively (5.1.2). For the hydrogenic species corresponding to the prefill gas, the initial profile shape of the plasma density is specified arbitrarily, while the profile of the wall concentration is given by the initial wall deposition profile as described above. Thus, the initial conditions for the prefill species are

$$
\begin{aligned}
\int^{a} n_{H j}\left(\rho, t_{0}\right) V_{\rho}^{i} d \rho & =f_{\mathrm{pl}} N_{\mathrm{pf}} \\
N_{w j} & =f_{\mathrm{wl}} f_{\mathrm{cx}}^{w} N_{\mathrm{pf}} \\
N_{L j}\left(t_{0}\right) & =f_{\mathrm{wl}} f_{\mathrm{cx}}^{L} N_{\mathrm{pf}}
\end{aligned}
$$

For the other hydrogenic-ion species (not prefilled), the initial values are set as described in the previous paragraph for a stand-alone simulation. Normally, the initial level of the other species is assumed to be very small.

The initial toroidal current profile $I\left(\rho, t_{0}\right)(2.1 .7)$ is determined by assuming that (1) the initial average toroidal electric field is flat $\left(\partial E_{z}\left(\rho, t_{0}\right) / \partial \rho=0\right)(2.1 .7)$ and (2) the total toroidal current $I_{0}\left(t_{0}\right)$ is specified. With these assumptions, the initial current profile is

$$
I\left(\rho, t_{0}\right)=I_{0}\left(t_{0}\right) \int_{0}^{r_{\rho}} \frac{1}{\eta_{\|}} r_{\rho}^{\prime} c_{\phi} d r_{\rho}^{\prime} / \int_{0}^{r_{a}} \frac{1}{\eta_{\|}} r_{\rho}^{\prime} c_{\phi} d r_{\rho}^{\prime}
$$


where the resistivity $\eta_{\|}(2.2 .8)$ and magnetic metric coefficient $c_{\phi}(6.4)$ are defined below.

\subsection{PLASMA AND TRANSPORT PARAMETERS}

\subsubsection{Plasma Parameters}

The electron density $n_{e}$ is obtained from charge neutrality by

$$
\begin{aligned}
n_{e} & =n_{H}+n_{\mathrm{He}} Z_{\mathrm{He}}+\sum_{j} n_{I j} Z_{j}+n_{b} \\
n_{H} & =\sum_{j} n_{H j}
\end{aligned}
$$

where $n_{H}$ is the total hydrogenic-ion density (2.1.3), $n_{\mathrm{He}}$ is the helium density (2.1.2), $n_{I j}$ is the impurity density (2.1.3), and $n_{b}$ is the injected fast-ion density (7.1). The effective $Z$ of the plasma, which appears, for example, in the plasma parallel resistivity, is

$$
Z_{\text {eff }}=\frac{1}{n_{e}-n_{b}}\left(n_{H}+n_{\mathrm{He}} Z_{\mathrm{He}}^{2}+\sum_{j} n_{I j}\left\langle Z_{j}^{2}\right\rangle\right)
$$

where the average charge state $Z_{j}$ is defined above (2.1.3). For the ion conductivity (2.2.6), a slightly different effective $Z$ that accounts for the difference between like and unlike particle collision frequencies is required and is given by

$$
Z_{\text {eff }}^{i}=\frac{1}{n_{H}}\left[n_{H}+\sqrt{2}\left(n_{\mathrm{He}} Z_{\mathrm{He}}^{2}+\sum_{j} n_{I j}\left\langle Z_{j}^{2}\right\rangle\right)\right]
$$

The mass-weighted $Z$ which appears in energy equilibration rates is given in terms of the ion masses $\left(m_{H j}, m_{\mathrm{He}}, m_{I j}\right)$ and the proton mass $m_{p}$ by

$$
[Z]=\frac{1}{n_{e}-n_{b}}\left(\sum_{j} n_{H j} \frac{m_{p}}{m_{H j}}+n_{\mathrm{He}} Z_{\mathrm{He}}^{2} \frac{m_{p}}{m_{\mathrm{He}}}+\sum_{j} n_{I j}\left\langle Z_{j}^{2}\right\rangle \frac{m_{p}}{m_{I j}}\right)
$$

The basic electron collision time [1] is

$$
\begin{aligned}
\tau_{e} & =\frac{3 m_{e}^{1 / 2} T_{e}^{3 / 2}}{4(2 \pi)^{1 / 2} e^{4} n_{e} \ln \Lambda} \\
\ln \Lambda & =23-\ln \frac{n_{e}^{1 / 2}\left(\mathrm{~cm}^{-3}\right)}{T_{e}(\mathrm{eV})}
\end{aligned}
$$

where $\ln \Lambda$ is the Coulomb logarithm. The electron collision frequency including impurities is

$$
\nu_{e}=Z_{\text {eff }} / \tau_{e}
$$

The collision frequency of hydrogenic-ion species $j$ is

$$
\nu_{H j}=\left(\frac{T_{e}}{T_{i}}\right)^{3 / 2}\left(\frac{m_{e}}{m_{H j}}\right)^{1 / 2}\left(\frac{n_{H}}{n_{e}}\right) \frac{Z_{e f f}^{i}}{\sqrt{2} \tau_{e}}
$$


The safety factor $q$ and the rotational transform $t$ are given by

$$
q=c_{\phi} \frac{r_{p}}{R_{0}} \frac{B_{T}}{B_{\theta}} \quad \text { and } \quad t=\frac{1}{q}
$$

in terms of the geometric factor $c_{\phi}(6.4)$, average poloidal magnetic field $B_{\theta}$ (2.1.7), and the toroidal magnetic field $B_{T}$ at major radius $R_{0}$.

The electron $(\alpha=e)$ and ion species $j(\alpha=H j)$ values of the toroidal gyroradius $\rho_{\alpha}$, thermal velocity $v_{\alpha}$, and collisionallity $\nu_{* \alpha}$ are

$$
\begin{aligned}
\rho_{\alpha} & =\frac{m_{\alpha} v_{\alpha}}{e B_{T}} ; \quad v_{\alpha}=\left(\frac{2 T_{\alpha}}{m_{\alpha}}\right)^{1 / 2} \\
\nu_{* \alpha} & =\frac{\sqrt{2} R_{0} q \nu_{\alpha}}{v_{\alpha} \epsilon^{3 / 2}} ; \quad \epsilon=\frac{\rho r_{s}}{R_{0}}
\end{aligned}
$$

\subsubsection{Hydrogen and Helium Ion Transport Coefficients}

The particle diffusivity used for most modeling is based on an anomalous, empirical diffusivity $D_{A}$,

$$
D_{A}\left(\mathrm{~cm}^{2} / \mathrm{ms}\right)=\frac{2 \times 10^{13}}{\bar{n}_{e}\left(\mathrm{~cm}^{-3}\right)}\left[2+20\left(\frac{\rho}{a}\right)^{2}\right]+10 \exp \left[-\left(a_{s}-\rho\right) / 0.1 a_{s}\right]
$$

where $\bar{n}_{e}$ is the model line-average electron density and $\rho=a_{s}$ is the contour separating the confined and scrape-off plasmas. The hydrogenic-ion and helium diffusivities are

$$
\begin{aligned}
D_{H j} & =C_{D}(t) D_{A} \\
D_{\mathrm{He}} & =D_{A}
\end{aligned}
$$

where the multiplier $C_{D}(t)$ is either held constant or determined by feedback (11.2).

The neoclassical radial convective velocity $v_{\text {pnch }}$ is given by

$$
v_{\text {pnch }}=-\frac{1.65 \epsilon^{1 / 2}}{\left(1.0+0.791 \nu_{* e}^{1 / 2}+0.56 \nu_{* e}\right)\left(1.0+0.51 \nu_{* e} \epsilon^{3 / 2}\right)} \frac{E_{z}}{B_{\theta}}
$$

where $\nu_{* e}$ is the electron collisionallity (2.2.1), $E_{z}$ is the toroidal electric field (2.1.7), and $B_{\theta}$ is an average poloidal magnetic field (2.1.7). The form used here for $v_{\text {pnch }}$ is given by Hinton and Hazeltine [4].

An anomalous inward convective velocity is required to model central peaking of the density profile. A given density profile shape $f(\rho)$ may be obtained by adjusting the inward convective velocity to give zero net radial particle flux, that is, to force $\Gamma_{H j}$ (2.1) to evolve in time to zero. The anomalous convective velocity is given by

$$
v_{\text {pnch }}=\frac{\left\langle(\nabla \rho)^{2}\right\rangle_{\psi}}{\langle|\nabla \rho|\rangle_{\psi}} D_{H j} \frac{1}{f(\rho)} \frac{d f(\rho)}{d \rho}
$$

This model will evolve the density profile shape to $f(\rho)$ on a time scale determined by the magnitude of $D_{H j}$. The resulting $n_{H j}$ profile will be somewhat broader than $f(\rho)$ because of (1) the source of ionized neutrals at the edge and (2) the flattening of the $n_{H j}$ profile inside $\rho_{q=1}$ by enhancement of $D_{H j}$ in this region (6.6.1). The enhancement is added after the anomalous $v_{\text {pnch }}$ is calculated. 


\subsubsection{Impurity Ion Transport Coefficients}

The neoclassical impurity transport coefficients $D_{H}, K_{j}$, and $H_{j}$ that appear in the neoclassical impurity flux (2.1.3) are

$$
\begin{aligned}
D_{H} & =q^{2} \rho_{H}^{2} \nu_{H} \\
\rho_{H} & =\frac{\bar{m} v_{H}}{e B_{T}} ; \quad v_{H}=\left(\frac{2 T_{i}}{\bar{m}}\right)^{1 / 2} ; \quad \bar{m}=\frac{1}{n_{H}} \sum_{j} n_{H j} m_{H j} \\
\nu_{H} & =\left(\frac{T_{e}}{T_{i}}\right)^{3 / 2}\left(\frac{m_{e}}{\bar{m}}\right)^{1 / 2} \frac{1}{\tau_{e}}
\end{aligned}
$$

where $\bar{m}$ is the average hydrogenic-ion mass, $B_{T}$ is the toroidal magnetic field, and the other plasma parameters are defined above (2.2.1). In addition,

$$
\begin{aligned}
K_{j} & =C_{1 j} \\
H_{j} & =C_{1 j}-C_{2 j} \\
C_{1 j} & =1.0-\frac{0.52 \alpha_{I j}}{0.59+\alpha_{I j}+1.34 \beta^{2}} \\
C_{2 j} & =1.5-\frac{0.29+1.20 \alpha_{H j}}{0.59+\alpha_{I j}+1.34 \beta^{2}} \\
\alpha_{I j} & =\frac{n_{I j}\left\langle Z_{j}^{2}\right\rangle}{n_{H}} \\
\beta & =\frac{v_{H}}{\nu_{H} q R_{0}}
\end{aligned}
$$

The form of the neoclassical coefficients used here is given by Hirshman and Sigmar [5].

The anomalous impurity diffusivity $D_{A I}(2.1 .3)$ is usually taken to be a constant of approximately $5 \times 10^{3} \mathrm{~cm}^{3} / \mathrm{s}$. The anomalous convective velocity is chosen to force the tota! impurity density profile toward an arbitrarily chosen shape $f_{I}(\rho)$. This is the same model used to obtain centrally peaked ion density profiles (2.2.2). The convective velocity is given by

$$
v_{A I}=\frac{\left\langle(\nabla \rho)^{2}\right\rangle_{\psi}}{\langle|\nabla \rho|\rangle_{\psi}} D_{A I} \frac{1}{f_{I}(\rho)} \frac{d f_{I}(\rho)}{d \rho}
$$

The impurity density profile will evolve toward $f_{l}(\rho)$ only if the anomalous impurity fluxes are much larger than the neoclassical fluxes.

\subsubsection{Perpendicular Viscosity}

The perpendicular ion viscosity is given by

$$
\begin{aligned}
\eta_{\perp} & =\sum_{j} m_{H j} n_{H j} f_{j} q^{2} \rho_{H j}^{2} \nu_{H j} \\
f_{j} & =\frac{0.2}{0.7+\left(\nu_{* H j} \epsilon^{3 / 2}\right)^{2}}+\frac{2.3 \nu_{* H j} \epsilon^{3 / 2}}{1+\nu_{* H j} \epsilon^{3 / 2}}+\epsilon^{-3 / 2}
\end{aligned}
$$


where the plasma parameters are defined above (2.2.1). The first term in $f_{j}$ is the neoclassical value given by Tsang and Frieman [6], the second term is a collisional regime modification suggested by Hogan [7], and the third term, which is the dominant term, is an empirical enhancement required to predict measured toroidal rotation velocities using the toroidal momentum equation (2.1.4).

\subsubsection{Electron Heat Conduction Coefficient}

The empirical model used for ohmic tokamak discharges is

$$
\chi_{e}\left(\mathrm{~cm}^{2} / \mathrm{ms}\right)=\frac{2 \times 10^{13}}{\bar{n}_{e}\left(\mathrm{~cm}^{-3}\right)}\left[2+20\left(\frac{\rho}{a}\right)^{2}\right]
$$

which approximates Alcator scaling.

For neutral-beam-heated discharges, additional heat conduction loss due to pressuredriven ballooning modes [8] may be included:

$$
\begin{aligned}
\chi_{e} & =1.5 v_{T_{e}} r_{s} \frac{q(\rho)}{S}\left(\frac{\beta_{0} q^{2}(\rho)}{\epsilon_{0}} \frac{L_{q}}{L_{p}}\right)^{3 / 2}\left[1+\left(\frac{\omega_{*}}{\gamma}\right)^{2}\right]^{-1 / 4} \\
v_{T_{e}} & =\left(\frac{k T_{e}(\rho)}{m_{e}}\right)^{1 / 2} \\
S & =\frac{\tau_{R}}{\tau_{H P}} \\
\tau_{R} & =\frac{\mu_{0} r_{s}^{2}}{\eta_{\|}(\rho)} \\
\tau_{H P} & =\frac{R_{0}}{v_{A}} \\
v_{A} & =\frac{B_{T}}{\left(4 \pi \sum_{j} m_{H j} n_{H j}\right)^{1 / 2}} \\
\beta_{0} & =\frac{p(0)}{B_{T}^{2} / 2 \mu_{0}} \\
\epsilon_{0} & =\frac{r_{s}}{R_{0}} \\
L_{q} & =\operatorname{abs}\left[\frac{\rho}{q(\rho)} \frac{\partial q}{\partial \rho}\right]^{-1} \\
L_{p} & =\operatorname{abs}\left[\frac{a_{s}}{p(0)} \frac{\partial p}{\partial \rho}\right]^{-1}
\end{aligned}
$$

where $p$ is the plasma pressure, $\eta_{\|}$is the resistivity (2.2.8), and other plasma parameters are defined above (2.2.1).

The diamagnetic drift, stabilization factor [9] in $\chi_{e}$ requires the growth rate $\gamma$, which is given by the solution of

$$
\gamma\left(\gamma^{2}+\omega_{*}^{2}\right)=\gamma_{0}^{3}
$$


where

$$
\begin{aligned}
\omega_{*} & =\frac{\langle n\rangle q(\rho)}{\rho / a_{s}} \frac{m_{e}}{m_{p}} \frac{v_{T_{e}}^{2}}{a_{s}^{2} \omega_{c i}} \frac{1}{L_{n}} \\
\gamma_{0}^{3} & =\frac{1}{S}\left[\frac{\langle n\rangle q(\rho)}{\rho / a_{s}} \frac{\beta_{0} q(\rho)}{\epsilon_{0} L_{p}}\right]^{2}\left(\frac{v_{A}}{R_{0}}\right)^{3} \\
\langle n\rangle & \simeq 5-10 \\
\omega_{c i} & =\frac{e B_{T}}{m_{p} c} \\
L_{n} & =\operatorname{abs}\left[\frac{a_{s}}{n_{e}(\rho)} \frac{\partial \dot{n}_{e}}{\partial \rho}\right]^{-1}
\end{aligned}
$$

The ballooning mode transport model applies for $\rho_{q=1}<\rho<a_{s}$.

The diagonal part of the neoclassical electron heat conduction [4] is given by

$$
\begin{aligned}
\chi_{e} & =\left(\epsilon^{-3 / 2} K_{22}\right) q^{2} \rho_{e}^{2} \nu_{e} \\
K_{22} & =2.55\left[\frac{0.45 \nu_{* e}\left(\epsilon^{3 / 2}\right)^{2}}{1.0+0.43 \nu_{* e} \epsilon^{3 / 2}}+\frac{1.0}{1.0+0.45 \nu_{* e}^{1 / 2}+0.43 \nu_{* e}}\right]
\end{aligned}
$$

where the plasma parameters are defined above (2:2.1).

\subsubsection{Ion Heat Conduction Coefficient}

The neoclassical coefficient is from Hinton and Hazeltine [4] and is given by

$$
\begin{aligned}
\chi_{H j} & =K_{H j} q^{2} \rho_{H j}^{2} \nu_{H j} \\
K_{H j} & =0.66\left(\frac{1.77 \nu_{* H j} \epsilon^{3 / 2}}{1.0+0.74 \nu_{* H j} \epsilon^{3 / 2}}+\frac{K_{2}^{*} \epsilon^{-3 / 2}}{1.0+1.03 \nu_{* H j}^{1 / 2}+0.31 \nu_{* H j}}\right)
\end{aligned}
$$

where the plasma parameters are defined above (2.2.1). The finite toroidicity correction factor $K_{2}^{*}$, which was unity in the original derivation, is a simplified form of the complete derivation of Chang and Hinton [10] and is

$$
K_{2}^{*}=1+2.85 \epsilon^{1 / 2}-2.33 \epsilon
$$

Ion heat transport due to ripple in the toroidal magnetic field is given by Uckan et al. [11] as

$$
\begin{aligned}
\chi_{i}^{\text {rip }} & =(0.1) 46.5 \delta_{R}^{3 / 2} \frac{G(\alpha)}{\nu_{i}} v_{\perp}^{2} \\
v_{\perp} & =\frac{v_{T_{i}}^{2}}{\omega_{B_{T}} R_{0}} ; \quad \omega_{B_{T}}=\frac{2 \pi \epsilon B_{T}}{m_{i}} \\
G(\alpha) & = \begin{cases}1-3 \alpha+2.8 \alpha^{2} & \alpha<\frac{1}{2} \\
0.38-0.36 \alpha & \frac{1}{2}<\alpha<1 \\
0.02 / \alpha^{2} & \alpha>1\end{cases} \\
\alpha=\frac{c}{q N_{c} \delta_{R}} ; \quad N_{0} & =\text { number of coils; } \quad \delta_{R}=\left(\frac{B_{\max } \cdots B_{\min }}{B_{\max }+B_{\min }}\right)
\end{aligned}
$$


The ripple $\delta_{R}$ is specified in the midplane between the plastna center and the outside (in major radius) plasma edge. The factor 0.1 that appears in $\chi_{i}^{\text {rip }}$ approximately accounts for the flux surface average of the ripple heat conduction loss.

\subsubsection{Helical Ripple Transport}

The nonambipolar fluxes of particles $\Gamma_{a}^{n a}$ and heat $q_{a}^{\text {na }}$ of plasma species $a$ due to the helical ripple are given by Hastings, Houlberg, and Shaing [12]:

$$
\begin{aligned}
\Gamma_{a}^{n a} & =-\epsilon_{t}^{2} \sqrt{\epsilon_{h}} v_{d_{a}}^{2} n_{a} \int_{0}^{\infty} d x x^{5 / 2} e^{-x} \frac{\tilde{\nu}_{a}(x) A_{a}(x)}{\omega_{a}^{2}(x)} . \\
\left(q_{a}^{n a}+\frac{5}{2} \Gamma_{a}^{n a} T_{a}\right) & =-\epsilon_{t}^{2} \sqrt{\epsilon_{h}} v_{d_{a}}^{2} n_{a} T_{a} \int_{0}^{\infty} d x x^{7 / 2} e^{-x} \frac{\tilde{\nu}_{a}(x) A_{a}(x)}{\omega_{a}^{2}(x)}
\end{aligned}
$$

where

$$
\begin{aligned}
A_{a}(x) & =\frac{1}{n_{a}} \frac{\partial n_{a}}{\partial \rho}+\frac{Z_{a} e}{T_{a}} \frac{\partial \Phi}{\partial \rho}+\left(x-\frac{3}{2}\right) \frac{1}{T_{a}} \frac{\partial T_{a}}{\partial \rho} \\
\tilde{\nu}_{a}(x) & =\frac{\nu_{a}}{\epsilon_{h} x^{3 / 2}} \\
x & =\frac{\epsilon}{T_{a}} \\
\omega_{a}^{2}(x) & =3 \ddot{\nu}_{a}^{2}(x)+\left(\epsilon_{t} / \epsilon_{h}\right)\left\{1.67\left(\omega_{E}+\omega_{B_{a}}\right)^{2}\right. \\
& \left.+\left(\frac{\epsilon_{t}}{\epsilon_{h}}\right)^{1 / 2}\left[\frac{\left(\omega_{B_{a}}\right)^{2}}{4}+0.6\left|\omega_{B_{a}}\right| \tilde{\nu}_{a}\left(\frac{\epsilon_{h}}{\epsilon_{t}}\right)^{3 / 2}\right]\right\} \\
\omega_{E} & =\frac{1}{r_{s} \rho B} \frac{\partial \Phi}{\partial r_{p}} \\
\omega_{B_{a}} & =-v_{d_{a}} \frac{\partial \epsilon_{h}}{\partial r_{\rho}} x \\
v_{d_{a}} & =\frac{T_{a}}{Z_{a} e B \rho r_{s}}
\end{aligned}
$$

The collision frequency $\nu_{a}$ of plasma species $a$ is given above (2.2.1). The toroidal and helical modulations of the magnetic field are given by $\epsilon_{t}$ and $\epsilon_{h}$, respectively (6.2). The radial potential $\Phi(\rho)$ is specified arbitrarily and does not automatically ensure that the neoclassical particle fluxes are ambipolar.

\subsubsection{Resistivity}

The parallel resistivity $\eta_{\|}[13]$ is given by

$$
\eta_{\|}=\eta_{0} \alpha_{z} \gamma_{T}
$$

where, in terms of $\bar{Z}=Z_{\text {eff }}$,

$$
\begin{aligned}
\eta_{0} & =\frac{m_{e}}{e^{2} n_{e} \tau_{e}} \\
\alpha_{z} & =\frac{\bar{Z}}{3.40}\left(\frac{2.67+\bar{Z}}{1.13+\bar{Z}}\right)
\end{aligned}
$$


and the resistivity enhancement $\gamma_{T}$ due to the fraction of trapped electrons $f_{T}$ is

$$
\begin{aligned}
\gamma_{T}^{-1} & =\left(1-\frac{f_{T}}{1+\xi \nu_{* e} / \bar{Z}}\right)\left(1-\frac{c_{R}(\bar{Z}) f_{T^{\prime}}}{1+\xi \nu_{* e} / \bar{Z}}\right) \\
f_{T} & =1-\frac{(1-\delta)^{2}\left(1-\delta^{2}\right)^{1 / 2}}{1+1.46 \delta^{1 / 2}} ; \quad \delta=\frac{r_{\rho}}{R_{0}} \\
\xi & =0.58+0.20 \bar{Z} \\
c_{R}(\bar{Z}) & =\frac{0.56}{\bar{Z}}\left(\frac{3.0-\bar{Z}}{3.0+\bar{Z}}\right)
\end{aligned}
$$

The plasma parameters are defined above (2.2.1).

\subsubsection{Radiation}

The radiated power $q_{\text {rad }}$ that appears in the electron temperature equation $(2.1 .5)$ is the sum of hydrogen bremsstrahlung radiation $\left(q_{\text {rad }}^{B}\right)$, impurity radiation from each impurity species $j\left(q_{\text {rad }}^{l j}\right)$, and an arbitrary amount of additional radiation $\left(q_{\mathrm{rad}}^{A}\right)$. Thus,

$$
q_{\mathrm{rad}}(\rho)=q_{\mathrm{rad}}^{B}(\rho)+\sum_{j} q_{\mathrm{rad}}^{I j}(\rho)+q_{\mathrm{rad}}^{A}(\rho)
$$

Radiation due to hydrogen bremsstrahlung is given by

$$
q_{\text {rad }}^{B}\left(\mathrm{~W} / \mathrm{cm}^{3}\right)=-1.6 \times 10^{-32} n_{e}\left(\mathrm{~cm}^{-3}\right) n_{H}\left(\mathrm{~cm}^{-3}\right) T_{e}(\mathrm{eV})^{1 / 2}
$$

If the impurity density $n_{I j}$ is specified arbitrarily or is governed by the average ion model, the radiation from impurity species $j$ is given by

$$
q_{\text {rad }}^{l j}(\rho)=-n_{e} n_{I j} l_{z}^{j}\left(T_{e}\right)
$$

where the coronal radiation rates $L_{z}$ are given by Post et al. [3].

When the impurity density $n_{I j}$ is governed by the multi-charge-state model, the radiation from impurity specios $j$ is given by

$$
q_{\text {rad }}^{l j}(\rho)=-n_{e} \sum_{l} n_{l j}^{q}\langle\sigma v\rangle_{e x}^{\ell}-n_{e} \sum_{q=0}^{Z-1} X_{R}^{q} n_{l, j}^{q+1}\langle\sigma v\rangle_{r}^{q}-1.6 \times 10^{-32} n_{e} T_{e}^{1 / 2} \sum_{q=1}^{Z} q^{2} n_{l, j}^{q}
$$

where the first term is due to line radiation summed over the strongest lines, the second term is recombination with the average radiated energy per recombination ovent given by $X_{R}^{q}$, and the third term is due to impurity bremsstrahlung.

The arbitrary radiation is given by

$$
q_{\text {radd }}^{A}(\rho)=-\frac{P_{\text {rad }}^{A}}{V_{p}} h_{\text {rad }}(\rho)
$$

where $P$ rad is the total power radiated in the arbitrary term and $V_{p}$ is the plasma volume. The normalized radiation profile is

$$
\begin{aligned}
h_{\operatorname{rad}}(\rho) & =\frac{\int(\rho)}{\left\langle\int(\rho)\right\rangle_{V}} \\
f(\rho) & =\mu_{c} T_{c}^{\prime}(\exp [-(\alpha-\rho) / \delta]
\end{aligned}
$$


where $\delta$ is an arbitrary width and the volume average $\langle\cdots\rangle_{V}$ is defined below (3.8).

The total arbitrary radiation power $P_{\text {rad }}^{A}$ is chosen in one of two ways. It may be specified as a fraction $f_{R}$ of the total power input to electrons

$$
P_{\mathrm{rad}}^{A}=f_{R} \int_{0}^{a}\left(q_{\mathrm{oh}}(\rho)+q_{\mathrm{inj}}^{e}(\rho)+q_{\mathrm{ic}}^{e}(\rho)+q_{\mathrm{fus}}^{e}(\rho)\right) V_{\rho}^{\prime} d \rho
$$

where the terms in the integrand are described above (2.1.5).

When modeling data from a plasma shot, the measured total radiation power $P_{\mathrm{rad}}^{M}(t)$ is sometimes used. In this case, the extra, arbitrary power at any time during the shot is adjusted so that the total power radiated by the $q_{\mathrm{rad}}$ term is $P_{\mathrm{rad}}^{M}$. Thus,

$$
P_{\mathrm{rad}}^{A}=P_{\mathrm{rad}}^{M}-P_{\mathrm{rad}}^{B}-P_{\mathrm{rad}}^{I}
$$

where $P_{\text {rad }}^{B}$ and $P_{\text {rad }}^{I}$ are the model values of the total radiated powers from bremsstrahlung and line radiation as calculated above.

\subsubsection{Radial Potential}

In an axisymmetric, toroidally rotating plasma, the plasma radial potential may be derived from the radial force balance of the plasma hydrogenic ions. This potential is

$$
\begin{aligned}
\Phi(\rho) & =-r_{s} \int_{\rho}^{a} \frac{\partial \Phi}{\partial \rho} d \rho, \quad \Phi(a) \equiv 0 \\
\frac{\partial \Phi}{\partial \rho} & =-\frac{1}{e n_{H} r_{s}} \frac{\partial p_{H}}{\partial \rho}-\frac{1}{c} v_{\phi} B_{\theta} \\
n_{H} & =\sum_{j} n_{H j} \\
p_{H} & =n_{H} T_{i}
\end{aligned}
$$

where $p_{H}$ is the plasma pressure, $B_{\theta}$ the poloidal magnetic field (2.1.7), and $v_{\phi}$ the toroidal rotation velocity (2.1.4). This form for $\Phi(\rho)$ is only valid when the poloidal rotation is completely damped. 


\section{NEUTRAL HYDROGEN TRANSPORT}

The transport of neutral hydrogen in the plasma is modeled with a two-species slab model. The model was developed by Audenaerde et al. [14], who describe the derivation of the governing equations and method of solution. The model has been expanded to allow two neutral species. Additional improvements include molecular sources (3.2.1), llmiter sources (3.2.2), momentum transport (3.4), wall sputtering (3.6.3), and wall deposition (3.7). The entire slab model is described here, as is the method of solution used in conjunction with the multispecies plasma transport model (3.8). The coupling of the neutral profiles to the plasma and wall transport models is described below (5.1).

\subsection{MULTIGENERATION CHARGE-EXCHANGE TRANSPORT}

The rate of creation of neutrals of species $j$ at position $x$ due to charge exchange is given by the solution of the Integral equation,

$$
s_{\mathrm{cx}}^{j}(x)=s_{0}^{j}(x)+\sum_{k} \int_{0}^{a} d x^{\prime} \nu_{i: \mathrm{x}}^{j k}\left(x, E_{T}\left(x^{\prime}\right)\right) s_{\mathrm{cx}}^{k}\left(x^{\prime}\right) \frac{1}{2} K_{2}^{k}\left(x, x^{\prime}, E_{T}\left(x^{\prime}\right)\right)
$$

where $s_{0}^{j}(x)$ is the source rate at position $x$ of neutrals of species $j$ due to the first charge exchange of neutrals emitted from the wall or limiter. This source has several contributions, which are described below (3.2).

The attenuation at position $x$ of a flux of neutrals of species $j$ emitted isotropically from position $x^{\prime}$ with energy $E$ is

$$
\begin{aligned}
h_{n}^{j}\left(x, x^{\prime}, E\right) & =-\frac{1}{\nu^{j}(x, E)} \frac{\partial}{\partial x} E_{n}\left(\beta^{j}\left(x, x^{\prime}, E\right)\right) \\
& =\frac{1}{v_{j}(E)} E_{n-1}^{\prime}\left(\beta^{j}\left(x, x^{\prime}, E\right)\right)
\end{aligned}
$$

'The ionization decrement between $x$ ' and $x$ is

$$
\beta^{j}\left(x, x^{\prime}, E\right)=\frac{1}{v_{j}\left(E^{\prime}\right)} \text { abs }\left[\int_{x^{\prime}}^{x} d x^{\prime \prime} \nu^{j}\left(x^{\prime \prime}, E^{\prime}\right)\right]
$$

where the total ionization frequency $\nu^{j}\left(x^{\prime}, E\right)$ at position $x$ of a neutral of species $j$ with energy $E$ is

$$
\nu^{j}(x, E)=\nu_{e}(x)+\sum_{k} \nu_{\mathrm{cx}}^{k j}(x, E)+\nu_{i}^{j}(x, E)
$$

The frequency of charge exchange at position $x$ of a neutral of species $k$ and encrgy $E$ with a plasma ion of species $j$ (producing a neutral of species $j$ ) is

$$
\nu_{c \mathrm{x}}^{j k}(x, E)=n_{H, j}(x)\langle\sigma v\rangle_{\mathrm{cx}}\left(v_{j}^{+}(x), v_{k}^{(j}(E)\right)
$$

The ionization frequency at position $x$ due to electron impart is

$$
u_{r}(x)=n_{r}(x)\langle\sigma u\rangle_{e}\left(T_{r}(x)\right)
$$


The ionization frequency at position $x$ due to ion impact of a neutral of species $j$ and energy $E$ by both plasma lon species is

$$
\nu_{i}^{j}(x, E)=\sum_{k} n_{H k}(x)\langle\sigma v\rangle_{i}\left(v_{k}^{+}(x), v_{j}^{0}(E)\right)
$$

The total impact lonization frequency at position $x$ of a neutral of species $j$ and energy $E$ is

$$
\nu_{\text {lon }}^{j}(x, E)=\nu_{e}(x)+\nu_{i}^{j}(x, E)
$$

The velocity of an ion of species $j$ and mass $m_{j}$ at position $x$ is assumed to be

$$
v_{j}^{+}(x)=\left(\frac{2 T_{i}(x)}{m_{j}}\right)^{1 / 2}
$$

The velocity of a neutral particle of species $j$, energy $E$, and mass $m_{j}$ is

$$
v_{j}^{0}(E)=\left(\frac{2 E}{m_{j}}\right)^{1 / 2}
$$

The energy of an ion with temperature $T_{i}$ is assumed to be

$$
E_{T}=\frac{3}{2} T_{i}
$$

The exponential integral that appears in the attenuation factor is

$$
E_{n}(\alpha)=\int_{1}^{\infty} d t \frac{e^{-\alpha t}}{t^{n}}
$$

where $n$ is the order of the integral $(n=0,1,2, \ldots)$.

\subsection{EDGE SOURCES}

The rate of creation at position $x$ of neutrals of species $j$ due to the first charge exchange of neutrals emitted from the wall and limiter contains several contributions, which are described in this section. The total creation rate is

$$
s_{0}^{j}(x)=s_{u A}^{j}(x)+s_{u m}^{j}(x)+s_{L}^{j}(x)+s_{R}^{j}(x)
$$

where $s_{u A}^{j}$ is due to atomic neutrals emitted from the wall (3.2.1), $s_{u m}^{j}$ results from the ionlzation and dissociation of wall-emitted molecules (3.2.1), $a_{L}^{i}$ is due to (atomic) neutrals emitted from the limiter (3.2.2), and $s_{R}^{j}$ is due to the charge-exchange efflux from the plasma that is reflected from the wall $(3.2 .3)$.

\subsubsection{Cold Wall Source}

The initial neutral flux incident on the plasma from the wall may be either atomic or molecular. 
Atomic flux The source at position $x$ of first-charge-exchange neutrals of species $j$ due to atomic neutrals emitted from the wall is

$$
s_{w A}^{j}(x)=\sum_{k} \Gamma_{w A}^{k} \nu_{\mathrm{dx}}^{j k}\left(x, E_{\mathrm{fc}}\right) 2 K_{3}^{k}\left(x, 0, E_{\mathrm{fc}}\right)
$$

where $\Gamma_{w A}^{k}$ is the flux of neutral species $k$ emitted inward from the wall with energy $E_{\mathrm{fc}}$ per atom.

Molecular flux The effects on the plesma that result frum introducing the wall flux as molecules rather than as atoms are described by Howe [15].

The source at position $x$ of first-charge-exchange neutrals of species $j$ due to atomic neutrals created by the ionization and dissociation of molecules emitted from the wall is

$$
\begin{aligned}
s_{u m}^{j}(x)= & \frac{1}{2} \sum_{k} \int_{0}^{a} d x^{\prime} s_{\mathrm{fc}}^{k}\left(x^{\prime}\right) \nu_{\mathrm{cx}}^{j k}\left(x, E_{\mathrm{fc}}\right)\left[K_{2}^{k}\left(x, x^{\prime}, E_{\mathrm{fc}}\right)\right. \\
& \left.+2 r_{\mathrm{fc}}^{k} E_{2}\left(\beta^{k}\left(x, 0, E_{\mathrm{fc}}\right)\right) K_{3}^{k}\left(0, x^{\prime}, E_{\mathrm{fc}}\right)\right]
\end{aligned}
$$

where $s_{\mathrm{fc}}^{k}(x)$ is the rate of creation of neutrals with energy $E_{\mathrm{fc}}$ from molecules of species $k$ at position $x$.

Atomic neutrals created from molecules at position $x^{\prime}$ may travel to position $x$ elther directly (first term in $s_{w m}$ ) or by first reflecting from the wall (second term in $s_{w m}$ ). The wa!l reflection coefficient for these neutrals is

$$
r_{\mathrm{fc}}^{k}=r_{R}^{k}\left(E_{\mathrm{fc}}\right)
$$

where $r_{R}$ is the wall reflection coefficient (3.2.3).

The creation rates of neutrals $\left(s_{\mathrm{fc}}\right)$ and hydrogenic ions $\left(s_{m+}\right)$ from molecules are

$$
\begin{aligned}
& s_{\mathrm{fc}}^{j}(x)=\Gamma_{u m}^{j} \frac{\nu_{m}(x)}{v_{j}\left(E_{m}\right)} E_{2}\left(\beta_{m}^{j}\left(x, E_{m}\right)\right)\left[\frac{2 \nu_{1}}{\nu_{1}+\nu_{2}}+\left(\frac{\nu_{2}}{\nu_{1}+\nu_{2}}\right)\left(\frac{\nu_{3}+2 \nu_{4}}{\nu_{3}+\nu_{4}}\right)\right] \\
& s_{m+}^{j}(x)=\Gamma_{u m m}^{j} \frac{\nu_{m}(x)}{v_{j}\left(E_{m}\right)} E_{2}\left(\beta_{m}^{j}\left(x, E_{m}\right)\right)\left(\frac{\nu_{2}}{\nu_{1}+\nu_{2}}\right)\left(\frac{\nu_{3}}{\nu_{3}+\nu_{4}}\right)
\end{aligned}
$$

where $\Gamma_{u m}^{j}$ is the flux of neutral particles of species $j$ emitted in molecular form (with energy $E_{H_{2}}$ per molecule) from the wall. 'The energy per neutral particle is $E_{m}^{\prime}=(1 / 2) E_{\mathrm{H}_{2}}$.

The attenuation decrement of a molecule between the wall $(x=0)$ and posit.on $x$ is

$$
\begin{aligned}
\beta_{m}^{i}(x, E) & =\frac{1}{v_{j}(E)} \text { abs }\left[\int_{0}^{x} d x^{\prime} \nu_{m}\left(x^{\prime}\right)\right] \\
\nu_{m}(x) & =\nu_{1}(x)+\nu_{2}(x)
\end{aligned}
$$

where $\nu_{m}$ is the frequency of molecular loss due to dissoclative excitation $\left(\nu_{1}\right)$ and ionization $\left(\nu_{2}\right)$.

The singly ionized molecules ( $\mathrm{H}_{2}^{+}$) created by molecular ionization are assumed to immediately dissociate either by excitation into an ion-neutral pair $(1 / 3)$ or by recombination into two neutrals ( $\left({ }^{\prime}\right)$. The rates for these two dissoriation processes are used to calculate the branching ratios in sife and $s_{m}+$ above.

The molecular reactions corresponding to anch rate coefficiont are 
$\begin{array}{lll}\langle\sigma v\rangle_{1} & \mathrm{H}_{2}+\mathrm{e} \rightarrow 2 \mathrm{H}+\mathrm{e} & \text { Dissociative excitation } \\ \langle\sigma v\rangle_{2} & \mathrm{H}_{2}+\mathrm{e} \rightarrow \mathrm{H}_{2}^{+}+2 \mathrm{e} & \text { Ionization } \\ \langle\sigma v\rangle_{3} & \mathrm{H}_{2}^{+}+\mathrm{e} \rightarrow \mathrm{H}^{+}+\mathrm{H}+\mathrm{e} & \text { Dissociative excitation } \\ \langle\sigma v\rangle_{4} & \mathrm{H}_{2}^{+}+\mathrm{e} \rightarrow 2 \mathrm{H} & \text { Dissociative recombination }\end{array}$

where the reaction frequency is $\nu_{k}=n_{e}\langle\sigma v\rangle_{k}$ for $k=1-4$. The rate coefficients are given by Freeman and Jones [16] and by Jones [17].

\subsubsection{Cold Limiter Source}

The source at position $x$ of first-charge-exchange neutrals of species $j$ due to atomic neutrals emitted from the limiter is

$$
\begin{gathered}
s_{L}^{j}(x)=\sum_{k} \Gamma_{L}^{k} \int_{0}^{x_{L}} d x^{\prime} h_{k}^{\ell}\left(x^{\prime}\right) \nu_{c \mathrm{x}}^{j k}\left(x, E_{L}\right)\left[f_{L}^{i} K_{2}^{k}\left(x, x^{\prime}, E_{L}\right)\right. \\
\left.+2 \int_{L}^{o} r_{L}^{k} E_{2}^{\prime}\left(\beta^{k}\left(x, 0, E_{L}\right)\right) K_{3}^{k}\left(0, x^{\prime}, E_{L}\right)\right]
\end{gathered}
$$

where $E_{L}$ is the energy of the emitted neutrals, which includes the energy gained from the limiter surface potential by the plasma lons (3.8).

Plasma ions incident on the limiter are assumed to be reemitted as atomic neutrals at the position of incidence. This neutral flux is introduced into the neutral transport model as a volume source with total flux for species $k$ of $\Gamma_{L}^{k}$. A fraction $f_{L}^{i}$ of the neutral source is emitted inward while the remaining fraction of the flux $f_{L}^{o}=1-f_{L}^{i}$ is emitted outward toward the wall. The normalized deposition profile $h_{k}^{\ell}\left(x^{\prime}\right)$ is derived from the profile of plasma ion flux to the limiter (3.8).

Neutrals emitted from the limiter at position $x^{\prime}$ may travel to position $x$ either directly (first term in $s_{L}$ ) or by first reffecting from the wall (second term in $s_{L}$ ). The wall reflection coefficient for these noutrals is

$$
r_{L}^{k}=r_{R}^{k}\left(E_{L}\right)
$$

where $r_{R}$ is the wall refledion coefficient (3.2.3).

\subsubsection{Reflected Source}

The source of first-charge-exchange neutrals of species $j$ due to the flux of neutrals reflected from the wall is

$$
s_{R}^{j}(x)=\sum_{k} \int_{0}^{\infty} d E \nu_{c \times}^{j k}(x, E) 2 h_{3}^{-k}(x, 0, E) \frac{\partial \Gamma_{R}^{k}(E)}{\partial E}
$$

where the integral is over the reflected-nentral energy spectrum.

The differential flux of reflected neutrals of species $j$ at energy $E$ is

$$
\frac{\partial I_{R}^{j}(E)}{\partial E^{\prime}}=\int_{0}^{a} d x^{\prime} \frac{\partial^{2} I_{R}^{j^{j}}\left(x^{\prime}, E\right)}{\partial x^{\prime} \partial E^{\prime}}
$$


The differential flux of reflected neutrals of species $j$ at energy $E$ due to reflection of neutrals creatad by charge exchange at position $x^{\prime}$ in the plasma with energy $E_{T}$ is

$$
\begin{aligned}
& \frac{\partial^{2} \Gamma_{R}^{j}\left(x^{\prime}, E\right)}{\partial x^{\prime} \partial E}=\frac{1}{2} s_{\mathrm{cx}}^{j}\left(x^{\prime}\right)\left\{E_{2}\left[\beta^{j}\left(0, x^{\prime}, E_{T}\left(x^{\prime}\right)\right)\right]\right. \\
& \left.\quad-\left[1-r_{R}^{j}\left(E_{T}\left(x^{\prime}\right)\right)\right] E_{3}\left[\beta^{j}\left(0, x^{\prime}, E_{T}\left(x^{\prime}\right)\right)\right]\right\} \frac{1}{E_{T}\left(x^{\prime}\right)} f\left(E, E_{T}\left(x^{\prime}\right)\right)
\end{aligned}
$$

Here, $r_{R}^{j}(E)$ is the particle reflection coefficient for normal incidence of a neutral at energy $E$ as given by Oen and Robinson [18].

For a neutral particle flux incident on the wall at energy $E^{\prime}, f\left(E, E^{\prime}\right)$ is the energy distribution of the reflected flux per unit incident flux, where $E$ is the reflected energy. The reflected spectrum is normalized so that

$$
\frac{1}{E^{\prime}} \int_{0}^{\infty} f\left(E, E^{\prime}\right) d E=1
$$

for all $E^{\prime}$. If we assume that the reflected flux is uniformly distributed in energy up to the incident energy, then

$$
f\left(E, E^{\prime}\right)= \begin{cases}1, & E \leq E^{\prime} \\ 0, & E>E^{\prime}\end{cases}
$$

A more realistic model is obtained from a fit to the reflection histograms of Oen and Robinson [18] and to the reflected spectra measured by Eckstein and Verbeek [19]. The normalized spectrum for $E \leq E^{\prime}$ is

$$
f\left(E, E^{\prime}\right)=\frac{1}{\ln \left[1+\left(E^{\prime} / E_{p}\right)^{2}\right]} \frac{2 E^{\prime} E}{E_{p}^{2}+E^{2}}
$$

where $f\left(E^{\prime}, E^{\prime}\right)=0$ for $E>E^{\prime}$. The energy $E_{p}$ depends on the wall material.

\subsection{PLASMA ION SOURCE (SLAB)}

The source of plasma ions of species $j$ at position $x$ due to impact ionization and charge exchange $\left(s_{+}^{j}\right)$ is

$$
\begin{aligned}
& s_{+}^{j}(x)+s_{\mathrm{cx}}^{j}(x)=\Gamma_{w A}^{j} \nu^{j}\left(x, E_{\mathrm{fc}}\right) 2 K_{3}^{j}\left(x, 0, E_{\mathrm{fc}}\right)+s_{m+}^{j}(x) \\
& +\frac{1}{2} \int_{0}^{u} d x^{\prime} s_{\mathrm{fc}}^{j}\left(x^{\prime}\right) \nu^{j}\left(x, E_{\mathrm{fc}}\right)\left[K_{2}^{j}\left(x, x^{\prime}, E_{\mathrm{fc}}\right)\right. \\
& \left.\quad+2 r_{\mathrm{fc}}^{j} E_{2}\left(\beta^{j}\left(x, 0, E_{\mathrm{fc}}\right)\right) K_{3}^{j}\left(0, x^{\prime}, E_{\mathrm{fc}}\right)\right] \\
& +\Gamma_{L}^{j} \int_{0}^{x_{L}} d x^{\prime} h_{j}^{\ell}\left(x^{\prime}\right) \nu^{j}\left(x, E_{L}\right)\left[f_{L}^{j} K_{2}^{j}\left(x, x^{\prime}, E_{L}\right)\right. \\
& \left.\quad+2 f_{L}^{o} r_{L}^{j} E_{2}\left(\beta^{j}\left(x, 0, E_{L}\right)\right) K_{3}^{j}\left(0, x^{\prime}, E_{L}\right)\right] \\
& +\int_{0}^{a} d x^{\prime} \nu^{j}\left(x, E_{T}\left(x^{\prime}\right)\right) s_{\mathrm{cx}}^{j}\left(x^{\prime}\right) \frac{1}{2} K_{2}^{j}\left(x, x^{\prime}, E_{T}\left(x^{\prime}\right)\right) \\
& +\int_{0}^{\infty} d E^{\prime} \nu^{j}\left(x, E^{\prime}\right) 2 K_{3}^{j}\left(x, 0, E^{\prime}\right) \frac{\partial \Gamma_{R}^{j}\left(E^{\prime}\right)}{\partial E^{\prime}}
\end{aligned}
$$


where the terms are contributions due to atomic wall flux $\Gamma_{w A}$ (3.2.1), ions $\left(s_{m+}\right)$ and neutrals $\left(s_{\mathrm{fc}}\right)$ from molecules (3.2.1), flux $\Gamma_{L}$ emitted from the limiter (3.2.2), energetic neutral transport, and reflected neutrals.

The plasma neutral density of species $j$ is

$$
\begin{aligned}
n_{0}^{j}(x) & =\Gamma_{w A}^{j} 2 K_{3}^{r j}\left(x, 0, E_{\mathrm{fc}}\right) \\
& +\frac{1}{2} \int_{0}^{a} d x^{\prime} s_{\mathrm{fc}}^{j}\left(x^{\prime}\right)\left[K_{2}^{j}\left(x, x^{\prime}, E_{\mathrm{fc}}\right)+2 r_{\mathrm{fc}}^{j} E_{2}\left(\beta^{j}\left(x, 0, E_{\mathrm{fc}}\right)\right) K_{3}^{j}\left(0, x^{\prime}, E_{\mathrm{fc}}\right)\right] \\
& +\Gamma_{L}^{j} \int_{0}^{x_{L}} d x^{\prime} h_{j}^{\ell}\left(x^{\prime}\right)\left[f_{L}^{i} K_{2}^{j}\left(x, x^{\prime}, E_{L}\right)+2 f_{L}^{o} r_{L}^{j} E_{2}\left(\beta^{j}\left(x, 0, E_{L}\right)\right) K_{3}^{j}\left(0, x^{\prime}, E_{L}\right)\right] \\
& +\int_{0}^{a} d x^{\prime} s_{\mathrm{cx}}^{j}\left(x^{\prime}\right) \frac{1}{2} K_{2}^{j}\left(x, x^{\prime}, E_{\Gamma}\left(x^{\prime}\right)\right) \\
& +\int_{0}^{\infty} d E 2 K_{3}^{-j}(x, 0, E) \frac{\partial \Gamma_{R}^{j}(E)}{\partial E}
\end{aligned}
$$

where the terms contain contributions corresponding to those described above for the ion source $s_{+}^{j}$.

\subsection{TOROIDAL MOMENTUM DRAG (SLAB)}

The loss of momentum per unit volume due to neutral transport is

$$
\begin{aligned}
\dot{p}_{\mathrm{cx}}(x) & =v_{\phi}(x) \sum_{k}\left[s_{w A}^{k}(x)+s_{w m}^{k}(x)+s_{L}^{k}(x)\right] \\
& +\int_{0}^{a} d x^{\prime}\left[v_{\phi}(x)-v_{\phi}\left(x^{\prime}\right)\right] \sum_{j, k} \nu_{\mathrm{cx}}^{j k}\left(x, E_{T}\left(x^{\prime}\right)\right) s_{\mathrm{cx}}^{k}\left(x^{\prime}\right) \frac{1}{2} K_{2}^{k}\left(x, x^{\prime}, E_{T}\left(x^{\prime}\right)\right) \\
& -\int_{0}^{a} d x^{\prime} v_{\phi}\left(x^{\prime}\right) \sum_{j} \nu_{\mathrm{ion}}^{j}\left(x, E_{T}\left(x^{\prime}\right)\right) s_{\mathrm{cx}}^{j}\left(x^{\prime}\right) \frac{1}{2} K_{2}^{j}\left(x, x^{\prime}, E_{T}\left(x^{\prime}\right)\right) \\
& +\int_{0}^{\infty} d E v_{\phi}(x) \sum_{j, k} \nu_{\mathrm{cx}}^{j k}(x, E) 2 K_{3}^{k}(x, 0, E) \frac{\partial \Gamma_{R}^{k}(E)}{\partial E}
\end{aligned}
$$

where the plasma velocity $v_{\phi}(x)$ is directed parallel to the wall.

\subsection{PLASMA ENERGY LOSS (SLAB)}

The energy loss from the plasma ions due to transport of charge-exchange neutrals is

$$
\begin{aligned}
q_{\mathrm{cx}}(x) & =\left[\frac{3}{2} T_{i}(x)-E_{\mathrm{fc}}\right] \sum_{k}\left[s_{u, A}^{k}(x)+s_{w m}^{k}(x)\right] \\
& +\left[\frac{3}{2} T_{i}(x)-E_{L}\right] \sum_{k} s_{L}^{k}(x) \\
& +\int_{0}^{a} d x^{\prime} \frac{3}{2}\left[T_{i}(x)-T_{i}\left(x^{\prime}\right)\right] \sum_{j, k} \nu_{\mathrm{cx}}^{j k}\left(x, E_{T}\left(x^{\prime}\right)\right) s_{\mathrm{cx}}^{k}\left(x^{\prime}\right) \frac{1}{2} K_{2}^{k}\left(x, x^{\prime}, E_{T}\left(x^{\prime}\right)\right) \\
& +\int_{0}^{\infty} d E\left[\frac{3}{2} T_{i}(x)-E\right] \sum_{j, k} \nu_{\mathrm{cx}}^{j k}(x, E) 2 K_{3}^{k}(x, 0, E) \frac{\partial \Gamma_{R}^{k}(E)}{\partial E}
\end{aligned}
$$


where each term is described after the ion source equation above (3.3).

The energy regained by the plasma ions due to ionization of neutrals is

$$
\begin{aligned}
q_{i}(x)= & \sum_{k} q_{i}^{k}(x) \\
q_{i}^{j}(x)= & E_{\mathrm{fc}} \Gamma_{w A}^{j} \nu_{\mathrm{ion}}^{j}\left(x, E_{\mathrm{fc}}\right) 2 K_{3}^{j}\left(x, 0, E_{\mathrm{fc}}\right)+E_{\mathrm{fc}} s_{m+}^{j}(x) \\
+ & \frac{1}{2} \int_{0}^{a} d x^{\prime} E_{\mathrm{fc}} s_{\mathrm{fc}}^{j}\left(x^{\prime}\right) \nu_{\mathrm{ion}}^{j}\left(x, E_{\mathrm{fc}}\right)\left[K_{2}^{j}\left(x, x^{\prime}, E_{\mathrm{fc}}\right)\right. \\
& \left.+2 r_{\mathrm{fc}}^{j} E_{2}\left(\beta^{j}\left(x, 0, E_{\mathrm{fc}}\right)\right) K_{3}^{j}\left(0, x^{\prime}, E_{\mathrm{fc}}\right)\right] \\
+ & \Gamma_{L}^{j} \int_{0}^{x_{L}} d x^{\prime} E_{L} h_{j}^{\ell}\left(x^{\prime}\right) \nu_{\mathrm{ion}}^{j}\left(x, E_{L}\right)\left[f_{L}^{i} K_{2}^{j}\left(x, x^{\prime}, E_{L}\right)\right. \\
& \left.\quad+2 f_{L}^{o} r_{L}^{j} E_{2}\left(\beta^{j}\left(x, 0, E_{L}\right)\right) K_{3}^{j}\left(0, x^{\prime}, E_{L}\right)\right] \\
+ & \int_{0}^{a} d x^{\prime} \frac{3}{2} T_{i}\left(x^{\prime}\right) \nu_{\mathrm{ion}}^{j}\left(x, E_{T}\left(x^{\prime}\right)\right) s_{\mathrm{cx}}^{j}\left(x^{\prime}\right) \frac{1}{2} K_{2}^{j}\left(x, x^{\prime}, E_{T}\left(x^{\prime}\right)\right) \\
+ & \int_{0}^{\infty} d E E \nu_{\mathrm{ion}}^{j}(x, E) 2 K_{3}^{j}(x, 0, E) \frac{\partial \Gamma_{R}^{j}(E)}{\partial E}
\end{aligned}
$$

where the terms are described after the ion source equation above (3.3).

The total energy loss due to neutrals is the sum of $q_{\mathrm{cx}}$ and $q_{i}$.

\subsection{NEUTRAL FLUXES AT WALL}

\subsubsection{Particle Fluxes}

The flux of neutral particles of species $j$ incident on the wall due to neutrals created from molecular ionization is

$$
\Gamma_{\mathrm{fc}}^{j}=\int_{0}^{a} d x^{\prime} s_{\mathrm{fc}}^{j}\left(x^{\prime}\right) \frac{1}{2} E_{2}\left(\beta^{j}\left(0, x^{\prime}, E_{\mathrm{fc}}\right)\right)
$$

The wall flux due to neutrals emitted from the limiter is

$$
\Gamma_{L 0}^{j}=f_{L}^{o} \Gamma_{L}^{j} \int_{0}^{a} d x^{\prime} h_{j}^{\ell}\left(x^{\prime}\right) E_{2}\left(\beta^{j}\left(0, x^{\prime}, E_{L}\right)\right)
$$

The flux due to neutrals created within the plasma by charge exchange is

$$
\Gamma_{H}^{j}=\int_{0}^{a} d x^{\prime} s_{\mathrm{cx}}^{j}\left(x^{\prime}\right) \frac{1}{2} E_{2}\left[\beta^{j}\left(0, x^{\prime}, E_{T}\left(x^{\prime}\right)\right)\right]
$$

The flux of reflected energetic noutrals is

$$
\Gamma_{R}^{j}=\int_{0}^{\infty} d E \frac{\partial \Gamma_{R}^{j}(E)}{\partial E}
$$




\subsubsection{Energy Fluxes}

The flux of energy carried into the plasma by the neutrals of species $j$ emitted from the wall and limiter is

$$
\Phi_{w}^{j}=E_{\mathrm{fc}} \Gamma_{w A}^{j}+E_{L} \Gamma_{L}^{j}
$$

The energy flux carried to the wall by energetic charge-exchange neutrals is

$$
\Phi_{H}^{j}=\int_{0}^{a} d x^{\prime} \frac{3}{2} T_{i}\left(x^{\prime}\right) s_{\mathrm{cx}}^{j}\left(x^{\prime}\right) \frac{1}{2} E_{2}\left[\beta^{j}\left(0, x^{\prime}, E_{T}\left(x^{\prime}\right)\right)\right]
$$

The reflected energy flux carried into the plasma is

$$
\Phi_{R}^{j}=\int_{0}^{\infty} d E E \frac{\partial \Gamma_{R}^{j}(E)}{\partial E}
$$

\subsubsection{Sputtered Impurity Fluxes}

The flux of impurities sputtered from the wall by energetic charge-exchange neutrals created in the plasma is

$$
\Gamma_{s}^{I}=\sum_{k} \int_{0}^{a} d x^{\prime} s_{\mathrm{cx}}^{k}\left(x^{\prime}\right) \frac{1}{2} E_{1}\left[\beta^{k}\left(0, x^{\prime}, E_{T}\left(x^{\prime}\right)\right)\right] Y_{s}^{I}\left(E_{T}\left(x^{\prime}\right), m_{k}\right)
$$

where $Y_{s}^{I}$ is the normal-incidence sputter coefficient defined below (3.6.4).

\subsubsection{Sputter Yield Coefficient}

The average number of impurity atoms sputtered from the wall for each neutral incident normally on the wall is given by Smith [20] as

$$
\begin{aligned}
Y_{s}^{I}\left(E, m_{H}\right) & =\frac{20 Z_{I}^{2}}{E_{b}} \frac{m_{H}}{m_{I}} \frac{E^{\prime}}{\left(E^{\prime}+50 Z_{I}\right)^{2}} \\
E^{\prime} & =E-E_{t} \\
E_{t} & =\frac{\left(m_{H}+m_{I}\right)^{2}}{4 m_{H} m_{I}} E_{b}
\end{aligned}
$$

where $E$ is the energy of the neutral charge-exchange particle of mass $m_{H}, m_{I}$ and $Z_{I}$ are the mass and nuclear charge of the impurity (wall) atom, $E_{b}$ is the binding energy, $E_{t}$ is the sputtering threshold energy, and all energies are in electron volts. The sputter yield dependence on the angle of incidence $\theta_{i}$ of the neutral flux is is assumed to be $Y=$ $Y_{s}^{I} / \cos \left(\theta_{i}\right)$.

\subsection{HYDROGEN DEPOSITION PROFILE IN WALL}

The rate of deposition of nonreflected particles at depth $y$ in the wall is

$$
\begin{aligned}
g_{w}^{j}(y) & =\left(1-r_{\mathrm{fc}}^{j}\right) \Gamma_{\mathrm{fc}}^{j} h\left(y, E_{\mathrm{fc}}\right)+\left(1-r_{L}^{j}\right) \Gamma_{L 0}^{j} h\left(y, E_{L}\right) \\
& +\int_{0}^{a} d x^{\prime} s_{c \mathrm{x}}^{j}\left(x^{\prime}\right)\left[1-r_{R}^{j}\left(E_{T}^{\prime}\left(x^{\prime}\right)\right)\right] \frac{1}{2} E_{3}\left[\beta^{j}\left(0, x^{\prime}, E_{T}\left(x^{\prime}\right)\right)\right] h\left(y, E_{T}\left(x^{\prime}\right)\right)
\end{aligned}
$$


The first term is due to neutrals created by molecular ionization and dissociation within the plasma (3.2.1) that are incident on the wall with flux $\Gamma_{\mathrm{fc}}^{j}$ (3.6.1) and energy $E_{\mathrm{fc}}$. The second term represents neutrals emitted from the limiter (3.2.2) that contribute a flux $\Gamma_{L 0}^{j}(3.6 .1)$ to the wall at energy $E_{L}$. The wall reflection coefficients for these fluxes are $r_{\mathrm{fc}}^{j}$ (3.2.1) and $r_{L}^{j}$ (3.2.2), respectively, and the corresponding sticking coefficients are therefore $1-r_{\mathrm{fc}}^{j}$ and $1-r_{L}^{j}$. The last term in $g_{w}$ is due to energetic neutrals created in the plasma by charge exchange that reach the wall and are not reflected. The integral in this term is essentially over the energy spectrum of the charge-exchange flux incident on the wall.

The normalized deposition profile in the wall of neutrals incident at energy $E$ is given by $h(y, E)$. An approximate fit to the calculations of Oen and Robinson [21] for hydrogen on copper is [22]

$$
h(y, E)=\frac{27}{2} \frac{y^{2}}{\bar{y}^{3}} e^{-3 y / \bar{y}}
$$

where $h(y, E)$ is normalized so that

$$
\int_{0}^{\infty} h(y, E) d y=1
$$

for all $E$.

The average range $\bar{y}(E)$ for a particle incident at energy $E$ is

$$
\begin{aligned}
\bar{y}(E) & =f(\epsilon) R_{E} \\
R_{E} & =c E^{1 / 2}, \quad c=22.7 \AA /(\mathrm{eV})^{1 / 2} \\
\epsilon & =\frac{E}{E_{\ell}}, \quad E_{\ell}=3.12 \mathrm{keV} \\
f(\epsilon) & =0.12+1.625 \times 10^{-2}\left(3+\log _{10} \epsilon\right)^{2}
\end{aligned}
$$

\subsection{NORMALIZED TOROIDAL PARAMETERS AND PROFILES}

For simulation of a plasma with two hydrogenic-ion species, the neutral transport solution is obtained separately for each species. An arbitrary total neutral flux $\Gamma_{0}$ is assumed incident on the plasma slab from the wall and limiter for ion species 1 , while the incident flux of ion species 2 is assumed to be zero. The resulting ionization and energy loss profiles and wall fluxes for both ion species are calculated. The same arbitrary flux is then introduced for ion species 2 (while the flux for ion species 1 is assumed to be zero), and the calculation is repeated. The total source profiles used in the plasma transport model are then formed at each time by a sum of the neutral profiles for each species. This procedure ensures particle conservation of each hydrogenic species in the plasma-wall transport model as the plasma. and wall models evolve in the time between recalculation of the neutral transport.

Let the neutral flux incident on the plasma slab of ion species $k$ : $(k=1$ or 2$)$ be $\Gamma_{0}$ and assume that the incident flux of the other ion species is zero. The fractions of flux $\Gamma_{0}$ that are incident from the limiter $\left(f_{L}\right)$ and from the wall $\left(f_{W}\right)$ are determined from the neutral fluxes of species $k$ that are recycled, injected, or desorbed from the limiter and wall in the plasma transport model at the time the neutral transport calculation is performed. These 
fractions are

$$
\begin{aligned}
f_{W} & =\frac{\dot{N}_{k}^{W}+\dot{N}_{k}^{G}+\dot{N}_{k}^{D}}{\dot{N}_{k}^{C}} \\
f_{L} & =\frac{\dot{N}_{k}^{L}}{\dot{N}_{k}^{C}}
\end{aligned}
$$

where the particle flow rates $\dot{N}$ are defined below (5.1.1).

The wall flux of neutral particles, emitted as either atoms or molecules, used in the neutral transport model above is

$$
\Gamma_{w A}^{k}+\Gamma_{w m}^{k}=f_{W} \Gamma_{n}
$$

where the wall flux is usually chosen to be either all atomic with energy $E_{\mathrm{fc}}$ or all molecular (3.2.1).

The flux of neutrals emitted from the limiter is

$$
\begin{aligned}
& \Gamma_{L}^{k}=f_{L} \Gamma_{0} \\
& E_{L}=E_{\mathrm{fc}}+e \phi_{L} / 2.0
\end{aligned}
$$

where the energy of the limiter-emitted neutrals $E_{L}$ includes the energy gained by the ions as they pass through the limiter potential $\phi_{L}$ (5.3.2).

The flux of neutrals from the limiter: is included in the slab neutral transport model as a volume source. The source profile is determined by the profile of parallel ion flow to the limiter. The normalized profile is

$$
h_{k}^{\ell}(x)=\frac{n_{H k}(\rho(x)) / \tau_{\| H k}}{\int_{0}^{x_{L}}\left(n_{H k} / \tau_{\| H k}\right) d x^{\prime}}
$$

where $\tau_{\| H k}$ is the parallel ion loss time (5.3.1) and the normalization extends over the width of the scrape-off $\left(x_{L}\right)$ in the slab.

The mapping between $x$ and the plasma coordinate $\rho$ is necessarily somewhat subjective because of the contorted shape of the plasma. The plasma temperature and density at a distance $x$ into the plasma slab from the plasma edge are given by the corresponding value of the plasma parameter on the flux surface for which $x(\rho)=r_{a}-r_{\rho}$, where $r_{\rho}(6.1 .6)$ is the radius of the equivolume toroid for surface $\rho$.

The flux of species $j$ deposited in the wall due to injection of flux $\Gamma_{0}$ of species $k$ into the plasma (as described above) is

$$
p_{j k}=\frac{\left(1-r_{\mathrm{fc}}^{j}\right) \Gamma_{\mathrm{fc}}^{j}+\left(1-r_{L}^{j}\right) \Gamma_{L 0}^{j}+\Gamma_{H}^{j}-\Gamma_{R}^{j}}{\Gamma_{T}^{k}}
$$

where $\Gamma_{T}^{k}=\Gamma_{0}$ and the wall-deposited flux is expressed as a fraction of $\Gamma_{0}$. There are two values $(j=1,2)$ of the wall pumping fraction $\left(p_{j k}\right)$ for each value of $k$ due to charge exchange between species in the plasma. The wall fluxes used to define $p_{j k}$ are defined above (3.6.1), and $r_{\mathrm{fc}}^{j}$ (3.2.1) and $r_{L}^{j}$ (3.2.2) are the reflection coefficients of cold neutrals. 
The normalized source profile for flasma ions of species $j$ due to an edge flux of species $k$ is

$$
h_{j k}^{p}(\rho)=\frac{s_{+}^{j}(x(\rho))}{\left\langle s_{+}^{j}(x(\rho))\right\rangle_{V}}
$$

where a neutral transport parameter on flux surface $\rho$ is given by the corresponding value of the parameter at slab distance $x(\rho)$ from the plasma edge. $s_{+}^{j}(x)$ is the ion source profile derived above (3.3).

The volume average of any parameter $X(\rho)$ is

$$
\langle X(\rho)\rangle_{V}=\frac{1}{V_{p}} \int_{0}^{a} X(\rho) V_{\rho}^{\prime} d \rho
$$

The total flux of impurities sputtered from the wall due to a flux $\Gamma_{0}$ of neutral ion species $k$ incident on the plasma is

$$
\bar{y}_{I k}=\frac{\Gamma_{s}^{I}}{\Gamma_{T}^{k}}
$$

where the impurity flux $\Gamma_{s}^{I}$ (3.6.3) is normalized to the incident neutral flux.

The normalized deposition profile of neutral species $j$ in the wall due to a flux $\Gamma_{0}$ of species $k$ incident on the plasma is

$$
h_{j k}^{w}(y)=\frac{g_{w}^{j}(y)}{\int_{0}^{\infty} g_{w}^{j}(y) d y}
$$

where $y$ is the distance into the wall and $g_{w}^{j}$ is derived above (3.7).

The momentum loss term for both ion species due to a flux $\Gamma_{0}$ of neutral species $k$ incident on the plasma edge is

$$
\dot{p}_{\mathrm{cx}, k}^{s}(\rho)=\dot{p}_{\mathrm{cx}}(x(\rho))
$$

where the slab value for the momentum loss $\dot{p}_{\mathrm{cx}}(x)$ is derived from the slab neutral transport model (3.4).

The energy loss term for both ion species due to a flux $\Gamma_{0}$ of neutral species $k$ incident on the plasma edge is

$$
q_{\mathrm{cx}, k}^{g}(\rho)=q_{\mathrm{cx}}(x(\rho))-q_{i}(x(\rho))
$$

where the slab profiles for charge-exchange energy transport $\left(q_{\mathrm{cx}}\right)$ and reionization $\left(q_{i}\right)$ are derived from the slab neutral transport model (3.5).

The total neutral density due to a flux $\Gamma_{0}$ of neutral species $k$ incident on the plasma edge is

$$
n_{0, k}^{s}(\rho)=\sum_{j} n_{0}^{j}(x(\rho))
$$

where the neutral profile $n_{0}^{j}(x)$ is derived from the slab neutral transport model (3.3). 


\section{WALL AND LIMITER TRANSPORT}

\subsection{WALL TRAPPING AND DIFFUSION}

The concentration of hydrogen in the wall evolves according to Fick's law including traps [23] and is governed by

$$
\begin{aligned}
\frac{\partial c_{j}(y, t)}{\partial t} & =\frac{\partial}{\partial i /}\left(D_{u, j} \frac{\partial c_{j}(y, t)}{\partial y}\right)+G_{j}(y, t)-\frac{\partial c_{T j}(y, t)}{\partial t} \\
\frac{\partial c_{T j}(y, t)}{\partial t} & =\frac{D_{w j}}{\lambda^{2} c_{t^{\prime}}} c_{j}(y, t) c_{T^{\prime}}(y, t)-\nu c_{T_{j} j}(y, t) \\
c_{T^{\prime} e}(y, t) & =c_{T^{\prime}}(y)-\sum_{i^{\prime}} c_{T^{\prime} j^{\prime}}(y, t)
\end{aligned}
$$

where $y$ is the distance into the wall from the surface. For hydrogenic-atom species $j, c_{j}$ is the mobile concentration, $c_{T j}$ is the trapped concentration, $C_{j}$ is the volume source due to nonreflected neutrals incident on t.le wall from the plasma $(5.1 .2)$, and $D_{w j}$ is the diffusivity (4.2). Also, $c_{T_{0}}$ is the total concentration of traps, $c_{T e}$ is the concentration of empty traps, $c_{w}$ is the metal concentration, $\lambda$ is the jump distance, and $\nu$ is the detrapping frequency (4.2).

\subsection{MATERIAL COEFFICIEN'TS}

The wall diffusivity of hydrogenic-atom species $j$ is

$$
D_{w j}=\frac{D_{0}}{\sqrt{m_{j}}} \exp \left(-Q_{D} / k T\right)
$$

where $D_{0}$ is the diffusivity pre-exponential, $Q_{D}$ is the migration energy, $T$ is the wall temperature (assumed constant), and $m_{j}$ is the atomic mass.

The detrapping frequency is

$$
\begin{aligned}
\nu & =\nu_{0} \exp \left[-\left(Q_{D}+E_{T}\right) / k T\right]+\nu_{I} \\
\nu_{I} & =\frac{\sigma_{I}}{A_{u}} \sum_{j, k} \dot{N}_{k}^{C} p_{j k}
\end{aligned}
$$

where $\nu_{0}$ is the pre-exponential and $E_{T}$ is the trap binding energy. The empirical detrapping frequency $\nu_{I}$ is an empirical detrapping cross section $\sigma_{l}$ times the total flux of neutrals incident on the wall (5.1.1).

\subsection{SURFACE BOUNDARY CONDITIONS}

The flux emitted from the surface is given by a balance between recombinative desorption and outward diffusion from the bulk to the surface of the wall [24]. The surface boundary condition is

$$
\left.D_{u, j} \frac{\partial c_{j}}{\partial y}\right|_{y=0}=2 k_{r} c_{j}^{s} \sum_{j^{\prime}} c^{s} j^{\prime}
$$


where $k_{r}$ is the recombination rate coefficlent including surface roughness (Howe and langley [25]) and $c_{j}^{s}=c_{j}(y=0)$ is the concentration at the surface. At the inner boundary,

$$
\left.\frac{\partial c_{j}}{\partial y}\right|_{y=y_{\max }}=0
$$

\subsection{LIMITER TRANSPORT}

A simple point model is currently used to model the accumulation of plasma particles in the limiter. The number of particles of hydrogenic species $j$ in the limiter $N_{L j}$ is governed by

$$
\frac{d N_{L j}}{d t}=\left(1-r_{L}\right) \dot{N}_{L j}^{+}+\dot{N}_{L j}^{0}-\frac{N_{L j}}{\tau_{L}}
$$

where the source terms are due to ions and neutrals, and the loss term represents desorption at an arbitrary rate specified by the limiter confinement time $\tau_{L}$.

The ion source term arises from the fraction of the ion flux incident on the limiter that is not directly reflected; $r_{L}$ is the limiter reflection coefficient for lons. The total rate of incidence of hydrogenic ions of species $j$ on the limiter or divertor due to parallel plasma loss in the scrape-off is

$$
\dot{N}_{L j}^{+}=\int_{a_{s}}^{a_{d}} \frac{n_{H j}}{\tau_{\| H j}} V_{\rho}^{\prime} d \rho
$$

The parallel loss time is $\tau_{\| H j}(5.3 .1)$, and the integral extends over the scrape-off volume enclosed between the inner scrape-off edge $\left(\rho=a_{s}\right)$ and the flux surface coinciding with the pump limiter duct leading edge $\left(\rho=a_{d}\right)$. If there is no limiter pumping, $a_{d}=a$ and the ion flux to the entire limiter is included. When limiter pumping is included $\left(a_{d}<a\right)$, ions incident on the limiter at radial distances greater than the leading edge of the pumping duct $\left(\rho>a_{d}\right)$ are completely pumped.

The neutral source $\dot{N}_{L j}^{0}$ arises from neutrals created in the plasma that are absorbed by the limiter. 'This source is given below $(5.1,2)$. 


\section{PLASMA-WALL INTERACTION}

\subsection{COUPLED PLASMA AND WALL HYDROGEN SOURCES}

The basic method for coupling the plasma, neutral, and wall particle transport models is described by Howe [22]. The complete method is described here.

\subsubsection{Neutral Source Fluxes at Surfaces}

The total rate of emission of neutral hydrogenic spectes $j$ from all surfaces is

$$
\dot{N}_{j}^{C}=\dot{N}_{j}^{W}+\dot{N}_{j}^{L}+\dot{N}_{j}^{G}+\dot{N}_{j}^{D}
$$

The rate of emission from the wall due to recycling of plasma lost to the wall is

$$
\dot{N}_{j}^{W}=-\left.R_{w}\left(V_{\rho}^{\prime} \mathrm{I}_{H j}\right)\right|_{\rho=a}
$$

where $R_{w}$ is the fraction of the ion flux incident on the wall that is reemitted into the plasma as a neutral flux and $\Gamma_{H j}$ is defined above (2.1.1).

The rate of emission from the limiter or divertor is given by one of two models. If the absorption and release of plasma and neutrals by the limiter are not explicitly calculated, then the limiter emission rate is

$$
\dot{N}_{j}^{L}=R_{L} \dot{N}_{L j}^{+}
$$

where $\dot{N}_{L j}^{+}$is the total ion flux of hydrogen species $j$ incident on the limiter $(4,4)$ and $R_{L}$ is the fraction of ions flowing to the limiter that is reemitted into the plasma as a neutral flux, $R_{L}$ may be a constant or may be controlled by feedback (11.3).

If the absorption and release of particles at the limiter are modeled as described above (4.4), then the emission rate of neutral hydrogenic specles $j$ from the limiter is

$$
\dot{N}_{j}^{L}=r_{L} \dot{N}_{L j}^{+}+\frac{N_{L j}}{\tau_{L}}
$$

The first term represents ions that are directly reflected from the limiter as neutrals with reflection coefficient $r_{L}$. The second term gives the rate of desorption from the limiter.

External fueling with gas $\left(S_{G_{i j}}\right)$ or continuous pellet injection $\left(S_{p, j}^{\prime}\right)$ produces an additional wall neutral emission rate of

$$
\dot{N}_{i j}^{G i}=S_{G i j}+S_{p i j}^{\prime}
$$

When the wall diffusion and trapping model $(1,1,4.2)$ is used, the rate of emission from the wall due to recombination is

$$
\dot{N}_{j}^{P}=2 k_{r} A_{w} c_{j}^{y} \sum_{j^{\prime}} c_{j^{\prime}}^{q}
$$

where $k_{r}$ is the recombination rate coefficient, $A_{w}$ is the wall area, and $c_{j}^{s}=c_{j}(y=0)$ is the particle concentration at the surface of the wall. 
If the wall diffusion and trapping model is not used, complete desorption from the wall is obtained by letting the rate of desorption equal the rate of incidence of nonreflected neutrals on the wall from the plasma. For a plasma with two hydrogenic-lon species, let the two species be denoted by subscripts $j$ and $k$. Then the desorbed emlssion rate of species $j$ is

$$
\begin{aligned}
\dot{N}_{j}^{D} & =f_{c x}^{u} \frac{\dot{N}_{j}^{P}\left(1-p_{k k}-d\right)+\dot{N}_{k}^{P} p_{j k}}{d} \\
\dot{N}_{j}^{P} & =\dot{N}_{j}^{L}+\dot{N}_{j}^{W}+\dot{N}_{j}^{C} \\
d & =\left(1-p_{j j}\right)\left(1-p_{k k}\right)-p_{j k} p_{k, j}
\end{aligned}
$$

where the wall pumping fraction $p_{j k}$ is defined above (3.8) and $f_{\mathrm{c} x}^{w}$ is defined below (5.1.2). The complete-desorption model is usually used for long-pulse simulations where the wall is expected to be saturated.

The general recycling model described above does not apply for constant plasma density. Instead, the neutral source rate is determined from an arbitrarlly set global particle confinement time $\tau_{p}^{z}$. The total rate of emission of neutrals of species $j$ from all surfaces for constant plasma density is given by $\dot{N}_{j}^{C}=\dot{N}_{j}^{W}$ where

$$
\begin{aligned}
& \dot{N}_{j}^{W}=\frac{1}{\tau_{p}^{y}} \int_{0}^{a} n_{H j}\left(\rho^{\prime}\right) V_{\rho}^{\prime}\left(\rho^{\prime}\right) d \rho^{\prime} \\
& \dot{N}_{j}^{L}=\dot{N}_{j}^{G}=\dot{N}_{j}^{D}=0
\end{aligned}
$$

\subsubsection{Plasma, Wall, and Limiter Particle Sources}

The ion source term $S_{H j}$ that appears in the transport equation for hydrogentc ion density $n_{H j}(2.1 .1)$ is

$$
S_{H j}(\rho)=\frac{1}{V_{p}}\left(\dot{N}_{j}^{C} h_{j j}^{p}(\rho)-\sum_{k} \dot{N}_{k}^{C} p_{j k} h_{j k}^{p}(\rho)\right)
$$

where the total neutral emission rate $\left(\dot{N}_{j}^{C}\right)$ from the walls and limiter due to recycling and extermal fueling is given above $(5.1 .1)$. The normalized deposition profiles of the neutrals in the plasma $\left(h_{j k}^{p}\right)$ and the fractional pumping rates $p_{j k}$ are derived from the neut ral iransport model $(3,8)$.

The particle source term $C_{i,}^{\prime}$, which appears in the transport equation for the hydrogenic particle concentration $c_{j}$ in the wall $(h .1)$, is

$$
G_{j}(y)=\frac{f_{c x}^{u \prime}}{A_{w}} \sum_{k} \dot{N}_{k}^{C} p_{j k} h_{j k}^{u \prime}(y)
$$

where $A_{w}$ is the wall area and $\int_{c x}^{w \prime}$ is the fraction of the neutral outflux from the plasma that is deposited in the wall. The nomalized wall source deposition profile ho is derived from the neutral trunsport model ( 3.8$)$.

The rate of deposition of neutral particles of hydrogenic speries $j$ on the limiter (h.h) is

$$
\dot{N}_{l, j}^{(1}=\int_{l: x}^{l} \sum_{k} \dot{N}_{k}^{(i} \gamma_{j k}
$$


where $f_{\mathrm{c} x}^{L}=1-f_{\mathrm{c} x}^{u}$ is the fraction of the neutral outflux from the plasma that is deposited in the limiter.

This form for $S_{H j}, G_{j}$, and $\dot{N}_{L j}^{0}$ ensures particle conservation, since each noutral emitted from the wall or limiter is cleposited elther in the plasma as an lon or in the wall or the limiter as a neutral particle.

For constant plasma density where the goneral recycle model is not applicable, the lonkation source rate for hydrogenlc. Ion species $\mathrm{Hj}_{j}$ is

$$
S_{H, i}(\rho)=\frac{1}{V_{p}} \dot{N}_{j}^{C} h_{j, j}^{p}(\rho)
$$

The wall pumping fractions $p_{j k}$ are not included; this assumes that all charge-exchange particles that are not reflected from the wall are reemitted at the thermal energy.

\subsubsection{Plasma 'Toroidal Momentum Loss}

The toroldal momentum loss $\dot{p}_{\mathrm{cx}}$ due to neutral charge exchange, which appears in the toroldal velocity oquation $(2.1 .4)$, is

$$
\begin{aligned}
\dot{p}_{\mathrm{tx}}(\rho) & =-\sum_{k}\left(\Gamma_{k}^{\rho} / \Gamma_{0}\right) j_{\mathrm{cx}, k}^{y}(\rho) \\
\Gamma_{k}^{\rho} & =\dot{N}_{k}^{C} / A_{s p}
\end{aligned}
$$

where $\dot{N}_{k}^{C}$ is defined above $(5.1 .1)$ and $A_{s p}=(2 \pi)^{2} r_{a} R_{0}$ is approximately the plasma surface area. $\Gamma_{0}$ is the (arbitrary) edge neutral source flux used in the slab neutral transport model, and $\dot{p}_{c x, k}^{s}(3.8)$ is the slab value for the charge-exchange momentum loss.

\subsubsection{Plasma Energy Loss}

The energy loss $q_{c x}$ due to neutral charge exchange, which appears in the lon temperature equation (2.1.6), is

$$
\psi_{\mathrm{cx}}(\rho)=\sum_{k}\left(\Gamma_{k}^{\rho} / \Gamma_{0}\right) \psi_{\mathrm{c}: \mathrm{x}, k}^{s}(\rho)
$$

where $\Gamma_{k}^{p}$ and $\Gamma_{0}$ are defined above $(5.1 .3)$ and $q_{c x, k}^{s}(3.8)$ is the slab value for chargeexchange energy transport, including reionization.

The energy loss qion due to neutral tonization and radiation, which appears in the electron temperature equation (9.1.5), is

$$
r_{\text {lon }}(\rho)=-\frac{E_{\text {lon }}}{V_{p}} \sum_{k} \dot{N}_{k}^{C} h_{k k}^{p}(\rho)
$$

where $E_{\text {con }}$ is the energy lost per neutral ionization.

\subsubsection{Neutral Density}

The neutral density $n_{0}(\rho)$ in the plasma is

$$
n_{0}(\rho)=\sum_{k}\left(\mathrm{I}_{k}^{\rho} / \mathrm{I}_{0}^{\prime}\right) n_{0, k}^{s}(\rho)
$$

where $I_{k}^{o}$ and $I_{0}$ are defined above $(5.1 .3)$ and $n_{0, k}^{g}$ is the slab value for the neutral density $(3.8)$ 


\subsubsection{Average Recycle and Pumping Coefficient}

The global recycle coefficient is

$$
\begin{aligned}
\ddot{R} & =(1-\ddot{p})+\ddot{p} \gamma_{d} \\
\ddot{p} & =\frac{\sum_{j, k} \dot{N}_{k}^{G} p_{j k}}{\sum_{j} \dot{N}_{j}^{C}} \\
\gamma_{d} & =\frac{\sum_{j} \dot{N}_{j}^{p}}{\sum_{j, k} \dot{N}_{k}^{C} p_{j k}}
\end{aligned}
$$

where $\dot{N}_{j}^{C}, \dot{N}_{j}^{P}(5.1 .1)$, and $p_{j k}(8.8)$ are defined above, $\bar{p}$ is the total fraction of the incident neutral flux that is deposited in the wall, and $\gamma_{d}$ is the fractlun of this flux reemitted from the wall surface back into the plasma by recombinative desorption.

\subsection{IMPURITY SOURCES}

The impurity source term $S_{I j}$, which appears in the transport equations for impurity density $j\left(n_{I j}\right)$ (2.1.3), contains contrlbutions due to charge exchange neutral sputtering of the wall (5.2.1), lon sputtering of the llmiter (5.2.2), impurity recycling from the wall and limiter (5.2.3), and external injection from the wall $(5.2,4)$.

\subsubsection{Wall Charge-Exchange Neutral Sputtering}

The source of impurities due to sputtering of the wall by the energetic charge-exchange neutrals emitted from the plasma is

$$
S_{l, j}^{\prime}(\rho)=\frac{1}{V_{p}} \sum_{k} \dot{N}_{k}^{C} y_{I j, k} h_{l j}^{I}(\rho)
$$

where $\dot{N}_{k}^{C}$ is the cold neutral flux of liydrogenic specles $k$ incident on the plasma from the wall and limiter (5.1.1), $\hat{y}_{1 j, k}$ is the spittered flux of impurity species $j$ divided by the cold neutral flux of hydrogenic species $k$ (3.8), and $V_{p}$ is the plasma volume. The normalized impurity deposition proflle is

$$
\begin{aligned}
h_{I, j}^{I}(\rho) & =\frac{-\partial E_{3}\left(\beta^{I}\left(\rho, v_{l, j}\right)\right) / \partial r_{\rho}}{\left\langle-\partial E_{3}\left(\beta^{l}\left(\rho, v_{l, j}\right)\right) / \partial r_{\rho}\right\rangle_{V}} \\
\beta^{I}\left(\rho, v_{l j}\right) & =\frac{1}{v_{l, j}} \int_{v_{p}}^{r_{a}} n_{e}\left(\rho^{\prime}\right)\langle\sigma v\rangle_{I} d v_{\rho}^{\prime} \\
v_{l, j} & =\left(\frac{2 v_{l, j}}{m_{l, j}}\right)^{1 / 2}
\end{aligned}
$$

where $t_{1 j}$ is the energy of the sputtered noutral impurity, $m_{1, j}$ is the mass, and $\langle\sigma v\rangle_{l}$ is the neutral-impurity ionization rale coeflicient. The exponential integral tis $(3.1)$ and volume integral $\langle\ldots\rangle_{\gamma}(3.8)$ are defined above. 


\subsubsection{Limiter Ion Sputtering}

The Impurity source due to sputtering of the limiter by plasma lons flowing parallel to fleld lines in the scrapo-off layer is

$$
\begin{aligned}
S_{l j}^{\prime}(\rho) & =\frac{1}{V_{p}} \dot{N}_{I_{j}}^{s} h_{I j}^{s}(\rho) \\
\dot{N}_{I j}^{s} & =\int_{a_{a}}^{a_{d}} s_{I j}(\rho) V_{\rho}^{\prime} d \rho \\
h_{l j}^{s}(\rho) & =\frac{s_{I j}(\rho)}{\left\langle s_{I j}(\rho)\right\rangle_{V}} \\
s_{I j}(\rho) & =\sum_{k} \frac{n_{H k}}{\tau_{\| H k}} Y_{s}^{I j}\left(E, m_{H k}\right), \quad a_{s}<\rho<a_{d} \\
& =0, \quad a_{d}<\rho<a \\
E & =T_{i}(\rho)+2 T_{e}(\rho)
\end{aligned}
$$

where the sputter yield coefficient for a hydrogenic ion of energy $E$ and mass $m_{H}, Y_{s}^{I}\left(E, m_{H}\right)$, is given above (3.6.4) and $n_{H k}$ and $\tau_{\| H k}$ are the density (2.1.1) and parallel loss time (5.3.1) of hydrogenic-ion species $k$. The sputtered impuritles are assumed to be deposited (ionized) on the same flux surface $\rho$ where they are sputtered. The separation of the source into the product of a normalized deposition profile $h_{I j}^{9}(\rho)$ and the total rate of sputtered impurity emission $\dot{N}_{i j}^{s}$ ensures impurity number conservation. Ions that enter the limiter pumping duct $\left(\rho>a_{d}\right)(5.1 .1)$ are assumed not to sputter impurities into the main plasma. $V_{p}$ is the plasma volume and the volume integral $\langle\ldots\rangle_{V}$ is defined above (3.8).

\subsubsection{Wall and Limiter Recycling}

The impurity source due to recycling from the wall of a fraction $R_{1 w}$ of the outflux of impurity species $j$ is

$$
S_{I j}^{\prime}(\rho)=-\frac{R_{l w}}{V_{p}}\left(V_{\rho}^{\prime} \Gamma_{I j}\right)_{\rho=a} h_{l j}^{I}(\rho)
$$

where $\Gamma_{I j}(2.1 .3)$ and the normalized deposition profile $h_{I j}^{I}(5.2 .1)$ are defined above.

The impurity source due to complete recycling of impurity species $j$ from the limiter is

$$
\begin{aligned}
S_{I j}(\rho) & =\frac{1}{V_{p}} \dot{N}_{I j}^{R} h_{I j}^{R}(\rho) \\
\dot{N}_{I j}^{R} & =\int_{a,}^{a} s_{I j}^{R}(\rho) V_{\rho}^{\prime} d \rho \\
h_{I j}^{R}(\rho) & =\frac{s_{I j}^{R}(\rho)}{\left\langle s_{l j}^{R}(\rho)\right\rangle_{V}} \\
s_{I j}^{R}(\rho) & =\frac{n_{I j}}{\tau_{\| I j}}
\end{aligned}
$$

where the impurity parallel loss time $\tau_{\| I j}$ is defined below (5.3.1). 'The recycled impurities are assumed to be deposited (ionized) on the same flux surface $\rho$ where they are incident 
on the limiter. The separation of the source into the product of a normallzed deposition proflle $h_{l j}^{R}(\rho)$ and the total rate of recycled impurity emission $\dot{N}_{I j}^{R}$ ensures impurity number conservation, $V_{p}$ is the plasma volume $(6.3)$ and the volume integral $\langle\ldots\rangle_{V}$ is deflned above $(3.8)$.

\subsubsection{External Source}

The impurity source due to an external source such as impurity injection or laser blowoff Is given by

$$
S_{I j}(\rho)=\frac{1}{V_{p}} \dot{N}_{I j}^{\text {ext }} h_{I j}^{I}(\rho)
$$

where $\dot{N}_{I j}^{e x t}$ is the time-dependent external source rate and $h_{I j}^{l}$ is the normalized deposition profle (5.2.1).

\subsection{PLASMA SCRAPE-OFF PARALLEL LOSS}

\subsubsection{Parallel Loss Time}

The parallel loss time of plasma species $\mu(\mu=H j, \mathrm{He}$, or $I j)$ in the scrape-off layer is based on the loss time given by Emmert et al. [26] and is

$$
\begin{aligned}
\tau_{\| \mu} & =\frac{\pi R_{0}}{2 \alpha \alpha v_{\mu}} \frac{L}{2 \pi R_{0}} ; \quad \alpha=\frac{1}{2 \sqrt{\pi}} \\
v_{\mu} & =\left(\frac{2 T_{i}}{m_{\mu}}\right)^{1 / 2}
\end{aligned}
$$

where $v_{\mu}$ is the thermal velocity and $m_{\mu}$ is the mass. The average toroidal length $L$ is

$$
\begin{aligned}
\frac{L}{2 \pi R_{0}} & =1 \quad \text { for a poloidal diaphragm limiter } \\
& =q E \quad \text { for a toroidal belt limiter } \\
& =\frac{\pi}{2}\left[2\left(\rho-a_{s}\right) / a_{s}\right]^{-1 / 2} \quad \text { for a vertical rail limiter } \\
& =\frac{L_{B}}{2 \pi R_{0}}\left(1+L_{B} / \lambda_{\mathrm{cx}}\right) \quad \text { for a bundle divertor with mirror ratio } R_{m} \\
L_{B} & =2 \pi R_{0} R_{m} q_{D}\left(a-a_{s}\right) /\left(\rho-a_{s}\right) \\
\lambda_{c \mathrm{x}} & =\frac{1}{n_{0}(\rho) \sigma_{\mathrm{cx}}\left(v_{H j}\right)}
\end{aligned}
$$

When modeling a tokamak with a given set of limiters, the parallel loss time is given by

$$
\tau_{\| \mu}=\frac{\pi^{3 / 2} R_{0} r_{s}}{v_{\mu} s(\rho)}
$$

where s( $\rho)$ is the poloidal are length of flux surface $p$ intersected by the limiters as shown in Fig. 1. s( $\rho)$ changes during the shot as the flux surfaces shift with respect to the fixed limiters. 
Helium recycling is included in the model by assuming that the helium ions that flow to the limiter are reemitted and ionized at the position of incidence. This reduces the effective parallel loss rate in the scrape-off by increasing the loss time $\tau_{\| \mathrm{He}}$. If the parallel loss time calculated above is denoted by $\tau_{\| \mathrm{He}}^{\prime}$, then

$$
\begin{aligned}
\tau_{|| \mathrm{He}} & =\frac{\tau_{\| \mathrm{He}}^{\prime}}{1-R_{\mathrm{He}}}, \quad a_{s}<\rho<a_{d} \\
& =\tau_{\mathrm{He}}^{\prime}, \quad a_{d}<\rho<a
\end{aligned}
$$

where $R_{\mathrm{He}}$ is the limiter recycle coefficient of helium ions. Notice that all helium ions incident on the limiter at positions outside the leading edge of the pump duct $\left(\rho=a_{d}\right)$ (5.1.1) are assumed to be pumped.

\subsubsection{Energy Loss Factor}

The enhancement to the parallel electron energy loss in the scrape-off due to the sheath potential at the limiter or collector plate is

$$
\gamma=\frac{1}{1-\sigma_{s}}+\frac{e \phi_{L}}{2 k T_{e}}
$$

where $\sigma_{s}$ is the secondary electron emission coefficient of the limiter (described below) and $\phi_{L}$ is the limiter sheath potential. There are two models for $\phi_{L}$.

If the electron and ion fluxes to the limiter are ambipolar at every point on the limiter surface, then $\phi_{L}$ varies across the limiter face and is given by [26]

$$
\frac{e \phi_{L}}{k T_{e}}=\frac{1}{2} \ln \left[\frac{1}{4} \frac{m_{p}}{m_{e}} \frac{T_{e}}{T_{i}}\left(1-\sigma_{s}\right)^{2}\right]
$$

If, instead, currents can flow within the limiter, then the electron and ion fluxes are not necessarily locally ambipolar [27], and the sheath potential adjusts to ensure that the limiter is globally ambipolar; that is, the total flux to the limiter face is ambipolar, and locally nonambipolar fluxes are equilibrated by currents flowing in the limiter from regions of excessive electron flux to regions of excessive ion flux. If the sheath potential is assumed to be constant as a function of position on the limiter face, then $\phi_{L}$ is determined from the integral condition:

$$
\begin{aligned}
\int_{a_{s}}^{a} \dot{n}_{H \|} V_{\rho}^{\prime} d \rho & =\int_{a_{s}}^{a} \frac{1}{2}\left(1-\sigma_{s}\right) \frac{n_{e}\left(T_{e} / T_{i}\right)^{1 / 2}}{\sum_{j} n_{H j}\left(m_{e} / m_{H j}\right)^{1 / 2}} \exp \left(e \phi_{L} / k T_{e}\right) \dot{n}_{H \|} V_{\rho}^{\prime} d \rho \\
\dot{n}_{H \|} & =\sum_{j} \frac{n_{H j}}{\tau_{\| H j}}
\end{aligned}
$$

The secondary electron emission coefficient is evaluated at $E=T_{e}$, where

$$
\sigma_{s}(E)=\frac{1}{2} \sigma_{\max } \frac{\ln \left[20\left(E / E_{\max }\right)+1\right]}{\left[\left(E / E_{\max }\right)+1\right]^{3 / 4}}
$$

and, for iron, $\sigma_{\max }=1.3, E_{\max }=400 \mathrm{eV}$. The space-charge upper limit on $\sigma_{s}$ is accounted for by imposing a limit of $\sigma_{s}(E) \equiv 0.85$ if $\sigma_{s}(E)>0.85$. 


\section{EQUILIBRIUM}

\subsection{FLUX SURFACE EQUATIONS}

A point on a flux surface is located by its Cartesian cylindrical coordinates $x_{i}=(R, \phi ; Z)$, where $R$ is the major radius, $\phi$ is the geometric toroidal angle and $Z$ is the distance above the midplane. The corresponding flux coordinate is $\alpha_{i}=(\rho, \theta, \xi)$, where $\rho$ is the flux surface label, $\theta$ is a poloidal angle, and $\xi$ is a toroidal angle. Flux surfaces are defined by the inverse representation

$$
\begin{aligned}
& R(\rho, \theta, \xi)=\sum_{m, n} R_{m, n}(\rho) \cos (m \theta-n \xi) \\
& Z(\rho, \theta, \xi)=\sum_{m, n} Z_{m, n}(\rho) \sin (m \theta-n \xi) \\
& \phi(\rho, \theta, \xi)=\xi
\end{aligned}
$$

The set of coefficients $R_{m, n}$ and $Z_{m, n}$ determines the flux surface geometry. In addition, a third set of coefficients $\lambda_{m, n}(6.2)$ is required to define the magnetic fields. The models used for the $R_{m, n}, Z_{m, n}$ coefficients are described in this section.

\subsubsection{Axisymmetric Surfaces}

For axisymmetric plasmas (tokamaks), a shifted-ellipse representation is given by

$$
\begin{aligned}
R_{0,0} & =R_{0}+\Delta(\rho) \\
Z_{0,0} & =0 \\
R_{1,0} & =-r_{\rho} \\
Z_{1,0} & =E r_{\rho}
\end{aligned}
$$

This model is described in detail below (6.5) and is shown in Fig. 2. The metric coefficients used in the plasma transport equations may be derived in closed form for this representation; the resulting coefficients are also given below (6.5). The shifted-ellipse model was used in the tokamak version of the transport model [28]. Note, however, that the radial variable $\rho$ is a normalized radius here, while $\rho$ was the actual midplane minor radius in the tokamak model. The parameter $\rho$ used previously has been replaced here by $r_{\rho}$.

\subsubsection{Model 3-D Surfaces}

A model 3-D flux surface representation is used for model development and for cases where detailed surfaces are not available. The flux surfaces are a set of concentric ellipses with elongation $E_{3 \mathrm{D}}$ that rotate poloidally as the toroidal angle increases. The moment 
coefficients for this model are

$$
\begin{aligned}
R_{0,0} & =R_{3 \mathrm{D}} \\
Z_{0,0} & =0 \\
R_{1,0} & =a_{3 \mathrm{D}} \rho \\
Z_{1,0} & =a_{3 \mathrm{D}} \rho \\
R_{1, n 3 \mathrm{D}} & =-f_{E} a_{3 \mathrm{D}} \rho \\
Z_{1, n 3 \mathrm{D}} & =f_{E} a_{3 \mathrm{D}} \rho \\
f_{E} & =\left(E_{3 \mathrm{D}}-1\right) /\left(E_{3 \mathrm{D}}+1\right)
\end{aligned}
$$

where the major radius $R_{3 \mathrm{D}}$, average minor radius $a_{3 \mathrm{D}}$, and the number of field periods $n_{3 \mathrm{D}}$ are specified arbitrarily. The radial variable $\rho$ is a normalized radius, where $\rho=1$ is the last closed surface. This representation is fixed in real space as the plasma evolves in time.

\subsubsection{Vacuum 3-D Surfaces}

For low-beta torsatron plasmas, the vacuum flux surfaces may be used. Starting with a filamentary model of the helical and poloidal coil currents, a fast field-line-following model [29] is used to map out a set of flux surfaces. The resulting surfaces are spectraliy resolved into the inverse representation described above (6.1) using a method [30] that selects the poloidal angle $\theta$ to give the smallest number of $R_{m, n}$ and $Z_{m, n}$ coefficients. Typical vacuum surfaces for the Advanced Toroidal Facility calculated with this method are shown in Fig. 3.

The radial variable $\rho$ is defined as

$$
\rho=\left(\frac{\Phi}{\Phi_{a}}\right)^{1 / 2}
$$

where $\Phi(\rho)$ is the vacuum toroidal magnetic flux enclosed by surface $\rho$ and $\Phi_{a}$ is the toroidal flux enclosed by the outermost surface $(\rho=1)$. The toroidal fluxes are calculated by the field-line-following model.

\subsubsection{Fixed-Boundary 3-D Equilibr.iurn}

For moderate to high beta, the plasma equilibrium may be calculated [31]. This calculation returns the $R_{m, n}, Z_{m, n}$, and $\lambda_{m, n}$ coefficients for a given plasma pressure profile. The boundary may be specified from the vacuum surface calculation (6.1.3).

\subsubsection{Scrape-Off Surfaces}

The last closed flux surface is $\rho=1$ for all of the surface representations described above. The treatment of the scrape-off $(\rho>1)$ in the shifted-ellipse axisymmetric representation is described below (6.5). To include a scrape-off model in the 3 -ID representations, the surfaces outside the last closed surface are assumed to be linear extrapolations of the inner surfaces. The outermost surface modeled is labeled $\rho=a$; for the region $1 \leq \rho \leq a$, the 
representation is

$$
\begin{aligned}
& R_{m, n}(\rho)=R_{m, n}(\rho=1)+\left.\frac{d R_{m, n}}{d \rho}\right|_{\rho=1}(\rho-1) \\
& Z_{m, n}(\rho)=Z_{m, n}(\rho=1)+\left.\frac{d Z_{m, n}}{d \rho}\right|_{\rho=1}(\rho-1)
\end{aligned}
$$

The resulting flux surfaces for the model 3-D representation (6.1.2) are shown in Fig. 4.

\subsubsection{Equivolume Average Surfaces}

Several models (for example, magnetic diffusion (2.1.7) and neutral beam deposition (7.2)) have not yet been formulated for the 3 -D flux surface representation. Average, axisymmetric surfaces are defined by

$$
\begin{aligned}
& R(\rho, \theta)=R_{0}+\Delta(\rho)-r_{\rho} \cos \theta \\
& Z(\rho, \theta)=\operatorname{Er}_{\rho} \sin \theta
\end{aligned}
$$

where, for surface $\rho$, the average surface is defined to contain the same volume as the actual 3-D surface.

The real and average surfaces are aligned at the toroidal position $\phi=0$ as follows. The major radii of the inner $\left(R_{\text {in }}\right)$ and outer $\left(R_{\text {out }}\right)$ intersections of surface $\rho$ with the midplane are given approximately by

$$
\begin{aligned}
R_{\text {in }}(\rho) & =\sum_{m, n}(-1)^{m} R_{m, n}(\rho) \\
R_{\text {out }}(\rho) & =\sum_{m, n} R_{m, n}(\rho) \\
R_{\text {mid }}(\rho) & =\frac{1}{2}\left(R_{\text {in }}(\rho)+R_{\text {out }}(\rho)\right)
\end{aligned}
$$

where $R_{\operatorname{mid}}(\rho)$ is the average major radius of each flux surface. The plasma major radius and flux surface shift for the average surfaces are

$$
R_{0}=R_{\text {mid }}(\rho=1), \quad \Delta(\rho)=R_{\text {mid }}(\rho)-R_{0}
$$

If $V(\rho)$ is the volume contained within surface $\rho$ (as defined below (6.3)), then the midplane minor radius of the average surface is

$$
r_{\rho}(\rho)=\left[\frac{V(\rho)}{2 \pi^{2} E\left(R_{0}+\Delta(\rho)\right)}\right]^{1 / 2}
$$

A comparison between the real and average flux surfaces is shown in Fig. 5. The average radius of the last closed surface $(\rho=1)$ is $r_{s}$ and of the outermost surface $(\rho=a)$ is $r_{a}$.

If the axisymmetric shifted-cllipse representation (6.5) is used, the average and actual surfaces coincide and $R_{0}, r_{\rho}$, and $\Delta(\rho)$ become the surface major radius, minor radius, and shift as defined below (0.5). 


\subsection{MAGNETIC FIELDS AND FLUXES}

If the magnetic field magnitude on flux surface $\rho$ is approximated by

$$
B(\rho, \theta, \phi)=B_{0}(\rho)\left[1+\epsilon_{t}(\rho) \cos \theta+\epsilon_{h}(\rho) \cos (m \theta-n \phi)+\cdots\right]
$$

where $m=2$ and $n=$ number of field periods, then $\epsilon_{t}$ is the toroidal ripple and $\epsilon_{h}$ is the helical ripple. For the vacuum surfaces (6.1.3), $B_{0}, \epsilon_{t}$, and $\epsilon_{h}$ are derived from field-line following for each surface.

\subsection{PLASMA TRANSPORT METRICS}

The flux surface average of a variable $X(\rho, \theta, \xi)$ is

$$
\langle X\rangle_{\psi}=\frac{1}{V_{\rho}^{\prime}(\rho)} \int_{0}^{2 \pi} d \xi \int_{0}^{2 \pi} d \theta \sqrt{g} X
$$

The plasma volume between $\rho$ and $\rho+d \rho$ is

$$
V_{\rho}^{\prime}(\rho)=\int_{0}^{2 \pi} d \xi \int_{0}^{2 \pi} d \theta \sqrt{g}
$$

and the volume contained within surface $\rho$ is

$$
V(\rho)=\int_{0}^{\rho} d \rho^{\prime} V_{\rho}^{\prime}\left(\rho^{\prime}\right)
$$

where $V_{p}=V(\rho=a)$ is the total plasma volume including the scrape-off.

The differential plasma volume is

$$
\begin{aligned}
\sqrt{g} & =R \operatorname{det}\left(G_{i j}\right) \\
G_{i j} & =\partial x_{i} / \partial \alpha_{j}
\end{aligned}
$$

where, as defined above $(6.1), x_{i}=(R, \phi, Z)$ is the cylindrical coordinate and $\alpha_{i}=(\rho, \theta, \xi)$ is the corresponding point in flux coordinates. Since the magnetic toroidal angle $\xi$ is defined to be the geometric toroidal angle $\phi, \sqrt{g}$ is given as in the axisymmetric case by

$$
\sqrt{g}=R\left(\frac{\partial R}{\partial \theta} \frac{\partial Z}{\partial \rho}-\frac{\partial R}{\partial \rho} \frac{\partial Z}{\partial \theta}\right)
$$

The metric coefficients are

$$
g_{i j}=\frac{\partial R}{\partial \alpha_{i}} \frac{\partial R}{\partial \alpha_{j}}+R^{2} \frac{\partial \phi}{\partial \alpha_{i}} \frac{\partial \phi}{\partial \alpha_{j}}+\frac{\partial Z}{\partial \alpha_{i}} \frac{\partial Z}{\partial \alpha_{j}}
$$

The transport metrics $\left\langle(\nabla \rho)^{2}\right\rangle_{\psi}$ and $\langle|\nabla \rho|\rangle_{\psi}$ are obtained by flux-surface averaging the local value

$$
(\nabla \rho)^{2}=\frac{1}{g}\left(g_{\theta \theta} g_{\xi \xi}-g_{\theta \xi}^{2}\right)
$$

where the flux surface average operator is defined above. While this average is performed numerically for the general 3-D flux surface representation, an analytical form is obtainable for the axisymmetric shifted-ellipse surfaces $(6.5)$. 


\subsection{MAGNETIC DIFFUSION METRICS}

The poloidal averages of the metric coefficients required for the magnetic diffusion equation (2.1.7) are

$$
\begin{aligned}
c_{\theta} & =\frac{R_{0} r_{\rho}^{\prime}}{r_{\rho}}\left\langle g_{\theta \theta} / \sqrt{g}\right\rangle_{\theta}=\frac{R_{0} r_{\rho}^{\prime}}{r_{\rho}}\left\langle\sqrt{g}(\nabla \rho)^{2} / R^{2}\right\rangle_{\theta} \\
& =\frac{R_{0}}{R_{M}} \frac{1}{E\left(\epsilon-\Delta_{\rho}\right)}\left[\left(\frac{\epsilon}{1+\delta_{\epsilon}}\right)\left(1+\frac{E^{2}}{\delta_{\epsilon}}\right)-\left(\frac{\Delta_{\rho}}{1+\delta_{\Delta^{\prime}}}\right)\left(1+\frac{E^{2}}{\delta_{\Delta^{\prime}}}\right)\right] . \\
c_{\phi} & =\frac{R_{0}}{r_{\rho}^{\prime} r_{\rho}}\left\langle\sqrt{g} / g_{\phi \phi}\right\rangle_{\theta}=\frac{R_{0}}{r_{\rho}^{\prime} r_{\rho}}\left\langle\sqrt{g} / R^{2}\right\rangle_{\theta} \\
& =\frac{R_{0}}{R_{M}} \frac{E}{\delta_{\epsilon}}\left(1-\frac{\epsilon \Delta_{\rho}}{1+\delta_{\epsilon}}\right) \\
\delta_{\epsilon} & =\left(1-\epsilon^{2}\right)^{1 / 2}, \quad \epsilon=\frac{r_{\rho}}{R}=\frac{r_{\rho}}{R_{0}+\Delta(\rho)} \\
\delta_{\Delta^{\prime}} & =\left(1-\Delta_{\rho}^{2}\right)^{1 / 2}, \quad \Delta_{\rho}=\frac{d \Delta}{d r_{\rho}}=\frac{\Delta^{\prime}}{r_{\rho}^{\prime}} \\
r_{\rho}^{\prime} & =\frac{d r_{\rho}}{d \rho}, \quad \Delta^{\prime}=\frac{d \Delta}{d \rho}
\end{aligned}
$$

\subsection{AXISYMMETRIC, SHIFTED, ELLIPTICAL SURFACES}

The plasma flux surfaces are assumed to be nested, elongated toroids. The equilibrium force balance is approximately satisfied by allowing each flux surface to shift in the major radius direction while assuming constant elongation for all surfaces. In cylindrical coordinates $(R, Z, \phi)$, where $R$ is the major radius, $Z$ the vertical distance above the torus midplane, and $\phi$ the toroidal angle, the equation of each surface is

$$
\begin{aligned}
R & =R_{M}(\rho)-r_{\rho} \cos \theta \\
Z & =E r_{\rho} \sin \theta \\
R_{M}(\rho) & =R_{0}+\Delta(\rho) ; \quad r_{\rho}=\rho r_{s} ; \quad r_{\rho}^{\prime}=r_{s}
\end{aligned}
$$

Here, $r_{\rho}$ is the midplane minor radius of the elliptical surface, $E$ is the elongation, $\theta$ is the poloidal angle, $R_{0}\left(r_{s}\right)$ is the major (minor midplane) radius of the surface $\left(\rho=a_{s}\right)$ that separates the confined and scrape-off plasmas, and $\Delta(\rho)$ is the shift, which is derived from the Grad-Shafranov equation. The entire plasma, including the scrape-off layer, is enclosed by the outer flux surface $\rho=a$. The geometry is shown in Fig. 2.

The matrix of metric coefficients that defines the coordinate transformation from cylin- 
drical to shifted-ellipse coordinates is

$$
\begin{aligned}
& g_{\rho \rho}=\left(\frac{\partial R}{\partial \rho}\right)^{2}+\left(\frac{\partial Z}{\partial \rho}\right)^{2}=\left(\Delta^{\prime}-r_{\rho}^{\prime} \cos \theta\right)^{2}+\left(E r_{\rho}^{\prime}\right)^{2} \sin ^{2} \theta \\
& g_{\rho \theta}=\frac{\partial R}{\partial \rho} \frac{\partial R}{\partial \theta}+\frac{\partial Z}{\partial \rho} \frac{\partial Z}{\partial \theta}=r_{\rho} \sin \theta\left[\Delta^{\prime}+\left(E^{2}-1\right) r_{\rho}^{\prime} \cos \theta\right] \\
& g_{\theta \theta}=\left(\frac{\partial R}{\partial \theta}\right)^{2}+\left(\frac{\partial Z}{\partial \theta}\right)^{2}=r_{\rho}^{2}\left(\sin ^{2} \theta+E^{2} \cos ^{2} \theta\right) \\
& g_{\phi \phi}=R^{2} \\
& g_{\rho \phi}=g_{\theta \phi}=0 \text { due to axisymmetry }
\end{aligned}
$$

The Jacobian is

$$
\begin{aligned}
g & =\operatorname{det}\left(g_{i j}\right)=g_{\phi \phi}\left(g_{\rho \rho} g_{\theta \theta}-g_{\rho \theta}^{2}\right) \\
\sqrt{g} & =R\left(\frac{\partial R}{\partial \theta} \frac{\partial Z}{\partial \rho}-\frac{\partial R}{\partial \rho} \frac{\partial Z}{\partial \theta}\right)=E R r_{\rho}\left(r_{\rho}^{\prime}-\Delta^{\prime} \cos \theta\right) \\
(\nabla \rho)^{2} & =\left(\frac{\partial \rho}{\partial R}\right)^{2}+\left(\frac{\partial \rho}{\partial Z}\right)^{2}=\frac{g_{\theta \theta} g_{\phi \phi}}{g}=\frac{\cos ^{2} \theta+\left(1 / E^{2}\right) \sin ^{2} \theta}{\left(r_{\rho}^{\prime}-\Delta^{\prime} \cos \theta\right)^{2}} .
\end{aligned}
$$

The coefficients that appear in the transport equations are poloidal averages of the metric coefficients $\left(g_{i j}\right)$. Tho poloidal average of a variable $X$ is

$$
\langle X\rangle_{\theta}=\frac{1}{2 \pi} \int_{0}^{2 \pi} X d \theta
$$

and the corresponding flux surface average is

$$
\langle X\rangle_{\psi}=\langle\sqrt{g} X\rangle_{\theta} /\langle\sqrt{g}\rangle_{\theta}
$$

One virtue of the shifted-ellipse, constant-elongation coordinate system is that all required poloidal averages may be obtained in closed form. Thus, the differential volume of a flux surface is

$$
V_{\rho}^{\prime}=(2 \pi)^{2}(\sqrt{g}\rangle_{\theta}=(2 \pi)^{2} E r_{\rho}^{\prime} r_{\rho}\left(R_{M}+\frac{\rho \Delta^{\prime}}{2}\right)
$$

where $\Delta^{\prime}=d \Delta(\rho) / d \rho$. The total volume enclosed by flux surface $\rho$ is $V(\rho)=2 \pi^{2} E r_{p}^{2} R_{M}$ and the volume of the entire plasma is $V_{p}=V(\rho=a)$.

The flux surface average of the transport term in the density, rotation, and temperature equations involves the integral of $(\nabla \rho)^{2}$ over the flux surface volume. The resulting factor is

$$
\begin{aligned}
V^{\prime}(\rho)\left\langle(\nabla \rho)^{2}\right\rangle_{\psi} & =(2 \pi)^{2}\left\langle\sqrt{g}(\nabla \rho)^{2}\right\rangle_{\theta} \\
& =(2 \pi)^{2} E \rho\left(\frac{R_{M}}{E^{2} \delta_{\Delta^{\prime}}}\left[1+\frac{E^{2}-1}{1+\delta_{\Delta^{\prime}}}\right]\right. \\
& \left.-\frac{\rho \Delta^{\prime}}{E^{2}} \frac{1}{\delta_{\Delta^{\prime}}\left(1+\delta_{\Delta^{\prime}}\right)}\left\{1+\frac{E^{2}-1}{4}\left[3+\left(\frac{\Delta_{\rho}}{1+\delta_{\Delta^{\prime}}}\right)^{2}\right]\right\}\right) \\
\delta_{\Delta^{\prime}} & =\left(1-\Delta_{\rho}^{2}\right)^{1 / 2}, \quad \Delta_{\rho}=\frac{\partial \Delta}{\partial r_{\rho}}=\frac{\iota^{\prime}}{r_{\rho}^{\prime}}=\frac{1}{r_{g}} \frac{d \Delta}{d \rho}
\end{aligned}
$$


The Integral of $\nabla \rho$ over a flux surface is approximated by

$$
\langle|\nabla \rho|\rangle_{\psi}=r_{\rho}^{\prime}\left\langle(\nabla \rho)^{2}\right\rangle_{\psi}
$$

The horizontal shift of the flux surfaces is derived from the Grad-Shafranov equation as described by Lao, Hirshman, and Wieland [32]. Thls representation is fairly accurate for small poloidal beta $\left(\beta_{p} \leq A / 2\right.$, where $A=R_{0} / r_{s}$ is the aspect ratio). The shift is defined relative to the surface $\left(\rho=a_{s}\right)$ that first touches a material surface (or the separatrix for a divertor); thus, $\Delta\left(\rho=a_{s}\right) \equiv 0$. This means that the shift is positive (outward) in the plasma and negative (inward) in the scrape-off layer. The shift $\Delta$ of surface $\rho$ is

$$
\begin{aligned}
\Delta(\rho) & =\frac{r_{s}^{2}}{R_{M}(0)}\left(\frac{4 E^{2}}{3 E^{2}+1}\right) \int_{\rho}^{a_{s}} \rho^{\prime} d \rho^{\prime}\left(\frac{\ddot{p}\left(\rho^{\prime}\right)-p\left(\rho^{\prime}\right)}{\tilde{B}_{p}^{2} / 8 \pi}\right) \\
& +\frac{r_{s}^{2}}{R_{M}(0)} \int_{\rho}^{a_{s}} \rho^{\prime} d \rho^{\prime}\left(\frac{1}{2} \tilde{\ell}_{i}\left(\rho^{\prime}\right)+\frac{1}{2} \frac{E^{2}-1}{3 E^{2}+1}\right)
\end{aligned}
$$

where the average poloidal field is

$$
\tilde{B}_{p}=\frac{2 E}{E^{2}+1} \frac{I(\rho)}{2 \pi \mu_{0} \rho r_{s}}
$$

The pressure is

$$
p=n_{e} T_{e}+n_{T} T_{i}+p_{b \perp}
$$

where $p_{b \perp}$ is the perpendicular fast ion pressure (7.1).

The average pressure is

$$
\ddot{p}(\rho)=\frac{2}{\rho^{2}} \int_{0}^{\rho} \rho^{\prime} d \rho^{\prime} p\left(\rho^{\prime}\right)
$$

the internal inductance is

$$
\tilde{\ell}_{i}(\rho)=\frac{2\left(E^{2}+1\right)}{3 E^{2}+1} \frac{1}{\tilde{B}_{p}^{2}(\rho)} \frac{2}{\rho^{2}} \int_{0}^{\rho} \rho^{\prime} d \rho^{\prime} \tilde{B}_{p}^{2}\left(\rho^{\prime}\right)
$$

and the major radius of the magnetic axis is $R_{M}(0)=R_{0}+\Delta(0)$.

The plasma equilibrium is recalculated whenever the plasma pressure or magnetic field changes sufficiently. Since the position of the confined-plasma edge $\left(R_{M}\left(\rho=a_{s}\right)=R_{0}\right)$ is assumed to be constant, large changes in plasma energy content or toroidal current due to major-radius compression are not included in the model. Therefore, only the plasma density is renormalized after the shift profile is recalculated in order to maintain particle conservation. The small resulting errors in the plasma energy density and current profile are usually unimportant. If we denote plasma parameters before recalculation of the shift profile with superscript $b$, then the corresponding densities in the new equilibrium are

$$
n_{\mu}(\rho)=\frac{V_{\rho}^{\prime b}(\rho)}{V_{\rho}^{\prime \prime}} n_{\mu}^{b}(\rho)
$$

where the renormalization is applied to the densition of hydrogenic ions $(\mu=H j$ ), helium ions $(\mu=H e)$, and impurity ions $(\mu=l j)$. 


\subsection{INTERNAL DISRUPTIONS}

\subsubsection{Transport Coefficient Enhancement}

When the discrete sawtooth model (6.6.2) is not used, enhanced transport due to in" ternal disruptions is simulated by increasing the plasma transport coefficients in the region where $q \leq 1$. The coefficients enhanced are $D_{H j}$ (2.2.2), $D_{H e}$ (2.2.2), $\eta_{\perp}$ (2.2.4), $\chi_{e}$ (2.2.5), and $\chi_{H j}$ (2.2.6). If the value of a particular coefficient $X$ before enhancement is $X_{0}$, the enhanced value is

$$
X=X_{0}+X_{\mathrm{st}}\left[1+\exp \left(\frac{q(\rho)-1}{0.05}\right)\right]^{-1}
$$

where the enhancement is performed for all radil; $q(\rho)$ is the safety factor (2.2.1), and the enhancement $X_{\text {st }}$ may be either a constant (usually $\approx 10^{4} \mathrm{~cm}^{2} / \mathrm{s}$ in present-day devices) or $0.01 D_{B}$, where $D_{B}=c k T_{e} / 16 e B_{T}$ is the Bohm diffusion coefficient.

Another model for transport enhancement is

$$
D_{\text {mhd }}=\frac{2\left(r_{s} \rho_{q=1}\right)^{2}}{\tau_{\text {st }}}
$$

where $\tau_{\mathrm{st}}$ is the sawtooth period and $\rho_{q=1}$ is the outermost flux surface for which $q=1$. An expression for $\tau_{\mathrm{st}}$ that agrees with experimental measurements from several ohmically heated tokamaks [33] is

where the resistive time is

$$
\tau_{\mathrm{st}}=3\left(\tau_{R}^{3} \tau_{A}^{2} \tau_{H}^{2}\right)^{1 / 7}
$$

$$
\tau_{R}=\frac{\mu_{0}\left(r_{s} \rho_{q=1}\right)^{2}}{\eta_{\|}(0)}
$$

the Alfvén time is

$$
\tau_{A}=\frac{R_{0}}{v_{A}}, \quad v_{A}=\frac{B_{T}}{\left(4 \pi \rho_{H}\right)^{1 / 2}}, \quad \rho_{H}=\sum_{j} m_{H j} n_{H j}
$$

and the central heating time is

$$
\tau_{H}=\frac{3}{2} \frac{n_{e}(0) T_{e}(0)}{E_{Z}(0) J_{\mathrm{oh}}(0)+q_{\mathrm{inj}}^{\mathrm{e}}(0)}
$$

The ohmic result for $\tau_{\mathrm{st}}$ has been extended here with the inclusion of $q_{\mathrm{nnj}}^{e}$ in $\tau_{H}$. For this model, the enhancement of any coefficient $X_{0}$ is given by

$$
X=X_{0}+D_{\text {mhd }}\left[1+\exp \left(\frac{\rho_{q=1}-\rho}{0.1 \rho_{q=1}}\right)\right]^{-1}
$$

The flattening of the toroidal current profile is simulated by modifying the parallel resistivity $\eta_{\|}$(2.2.8) inside the flux surface $\rho_{q=1}$ where $q=1$. The modified resistivity $\eta_{f}$ is used in the current evolution model (2.1.7) and is given by

$$
\begin{aligned}
\eta_{f}(\rho) & =\left(\frac{T_{e}\left(\rho_{q=1}\right)}{T_{e}^{\prime}(\rho)}\right)^{3 / 2} \eta_{\|}\left(\rho_{q=1}\right), \quad \rho<\rho_{q=1} \\
& =\eta_{\|}(\rho), \quad \rho>\rho_{q=1}
\end{aligned}
$$




\subsubsection{Sawtooth Model}

Let variables with a superscript zero be parameters immediately before the sawtooth and varlables with a superscript $f$ be the corresponding parameters immedialely following the sawtooth. A sawtooth is simulated by stopping the temporal evolution of the plasma parameters, flattening the plasma densitles, temperatures, and current density profiles as shown below, and starting the temporal evolution using the flattened profllos as initial conditions.

Let $\rho_{s}=\sqrt{2} \rho_{q=1}$, then the volume enclosed by the singular surface is

$$
V_{s}=\int_{0}^{\rho_{s}} V_{\rho}^{\prime} d \rho
$$

and the flattened values for $\rho \leq \rho_{s}$ are

$$
\begin{aligned}
n_{H j}^{f} & =\frac{1}{V_{s}} \int_{0}^{\rho_{s}} n_{H j}^{0} V_{\rho}^{\prime} d \rho \\
n_{I j}^{f} & =\frac{1}{V_{s}} \int_{0}^{\rho_{s}} n_{I j}^{0} V_{\rho}^{\prime} d \rho \\
n_{e}^{f} & =\sum_{j} n_{H j}^{f}+\sum_{j} Z_{j} n_{I, j}^{f}+n_{b} \\
T_{e}^{f} & =\frac{1}{V_{s} n_{e}^{f}} \int_{0}^{\rho_{s}} T_{e}^{0} n_{e}^{0} V_{\rho}^{\prime} d \rho \\
T_{i}^{f} & =\frac{1}{V_{s} n_{T}^{f}} \int_{0}^{\rho_{s}} T_{i}^{0} n_{T}^{0} V_{\rho}^{\prime} d \rho \\
n_{T^{\prime}} & =\sum_{j} n_{H, j}+\sum_{j} n_{I j} \\
I^{f}(\rho) & =J_{z}^{f} 2 \pi \int_{0}^{\rho} c_{\phi} \rho d \rho \\
J_{z}^{f} & =\frac{I^{0}\left(\rho_{s}\right)}{2 \pi \int_{0}^{\rho_{s}} c_{\phi} \rho d \rho}
\end{aligned}
$$




\section{NEUTRAL BEAM HEATING}

\subsection{PLASMA SOURCES}

Plasma sources of energy, momentum, and particles due to neutral beam Injectlon as well as fast-ion density and pressure are calculated for each beam energy component (full, onehalf, and one-third energy) and for each injector fast-neutral source. For energy component $j$ with energy $E_{j, k}\left(=E_{0 k} / j\right.$, where $E_{0 k}$ is the full injection energy) and particle current $I_{j, k}$ (where $k$ specifies the lon source), the flux-surfacenveraged fast-Ion source rate is

$$
\dot{n}_{j, k}=\frac{I_{j, k}}{e V_{p}} H_{j, k}(\rho)
$$

where $V_{p}$ is the plasma volume $(6.3)$ and $H_{j, k}(\rho)(7.2)$ is the normalized neutral beam deposition profile. Thermalization of the fast lons is modeled by the moments method (7.3.1), which gives the fractions of fast.ion energy, momentum, and particles deposited in the plasma electrons and lons. The total source terms are formed by summing the product of the fast-ion source rate and the approprlate moment over all beam components.

The heating source for electrons is

$$
q_{\text {inj }}^{e}(\rho)=\sum_{j, k} \dot{n}_{j, k} E_{j, k} C_{e_{j, k}}
$$

The heating source for lons is

$$
q_{\mathrm{inj}}^{i}(\rho)=\sum_{j, k} \dot{n}_{j, k} E_{j, k} G_{i j, k}+T_{i} S_{\mathrm{inj}}^{i}(\rho)
$$

The toroidal current density perturbation (including the shielding response of plasma electronsi) due to fast lons is

$$
\begin{aligned}
J_{b}(\rho) & =\sum_{j, k} \dot{n}_{j, k} Z_{f} e v_{j, k} \xi_{k} \tau_{s} K_{e, k}\left(1-Z_{f} / Z_{\text {eff }}\right) \\
v_{j, k} & =\left(\frac{2 E_{j, k}}{m_{f}}\right)^{1 / 2}, \quad \xi_{k}=\frac{\Theta_{k} R_{c, k}}{R_{0}+r_{\rho}}
\end{aligned}
$$

where, for source $k, \xi_{k}$ Is the cosine of the initial pitch angle and $\Theta_{k}=1(-1)$ for co-injection (counter-injection). The fast-ion density is

$$
n_{b}(\rho)=\sum_{j, k} \dot{n}_{j, k} \tau_{s} N_{j, k}
$$

The source of plasma ions due to thermalized fast lons is

$$
S_{\text {inj }}(\rho)=\sum_{j, k} \dot{n}_{j, k} p_{c \times x}\left(v_{T_{1}}\right)
$$

The source of toroldal momentum for the plasma (electrons and all ions) is

$$
\dot{p}_{\phi}^{T}(\rho)=\sum_{j, k} \dot{n}_{j, k} m_{\rho} v_{j, k} \xi_{k}\left(K_{e, k}+K_{i_{j, k}}\right)
$$


The source of toroldal momentum for the hydrogentc-lon plasma component is

$$
\dot{p}_{H}(\rho)=\sum_{j, k} \dot{n}_{j, k} m_{f} v_{j, k} \xi_{k} \Lambda_{H_{j, k}}
$$

The source of toroddal momentum for the plasma impuritles is

$$
\dot{p}_{I}(\rho)=\sum_{j, k} \dot{n}_{j, k} m \rho v_{j, k} \xi_{k} K_{I_{, k}}
$$

'The fast-ion perpendicular pressure is

$$
p_{b \perp}(\rho)=\sum_{j, k} \dot{n}_{j, k} b_{j, k} \tau_{s} P_{\perp_{j, k}}
$$

The fast-ion parallel pressure is

$$
p_{b \|}(\rho)=\sum_{j, k} \dot{n}_{j, k} E_{j, k} \tau_{s} P_{\|_{j, k}}
$$

\subsection{BEAM DEPOSITION}

The neutrail beam lonization and initial deposition model is based on the original model described by Rome et al. [34]. The present model departs from the original model by (1) oxcluding first-orbit effects and (2) including shifted, elongated flux surfaces.

\subsubsection{Diffuse-Beam Integration}

The neutral beam shape within the plasma is assumed to be a cylinder of radlus $r_{b}$. The langency major radius of the beam centerline for ton source $k$ is $R_{c}=R_{c, k}$. The normallzed deposition profile for flux surface $\rho$ is

$$
H_{j, k}(\rho)=H^{+}(\rho)+H^{-}(\rho)
$$

where the contribution from the outer $\left(\mathrm{H}^{+}\right)$and inner $\left(\mathrm{H}^{-}\right)$intersection of the beam with the flux surface is

$$
H^{ \pm}(\rho)=2 \int_{0}^{z_{u}} d z_{b} \int_{R_{L}}^{R_{\| t}^{ \pm}} d R_{b} J_{b}(\alpha) h^{ \pm}\left(\rho, R_{b}, z_{b}\right)
$$

The integration variables $R_{6}$ and $z_{6}$ are the major radius and distance from the midplane (in the beam tangency plane) of each differential beamlet. The integration in $R_{b}$ and $z_{b}$ extends over the beam cross section. 'The contribution of a beamlet at $\left(R_{b}, z_{b}\right)$ is $h^{ \pm}\left(\rho, R_{b}, z_{b}\right)$ and is given below (7.2.2). The integration limits

$$
\begin{aligned}
& z_{u}=\min \left[E_{i} r_{\rho}, r_{b}\right] \\
& R_{L}=R_{c}-\left(r_{b}^{2}-z_{b}^{2}\right)^{1 / 2} \\
& R_{u}^{t_{1}}=\min \left[R_{0}+\Delta(\rho) \pm\left(r_{p}^{2}-\left(z_{b} / l_{i}\right)^{2}\right)^{1 / 2}, R_{c}+\left(r_{b}^{2}-z_{b}^{2}\right)^{1 / 2}\right]
\end{aligned}
$$


onsure that only bemulets intersecting the given flux surface $\rho$ contribute to $H(\rho), H^{ \pm}(\rho)$ is zero If $R_{L}>R_{u}^{ \pm}$.

The current density distribution withln the beam cross section la

$$
\begin{aligned}
J_{b}(\alpha) & =\frac{1}{\pi \sigma^{2}}\left[1-\exp \left(-r_{b}^{2} / \sigma^{2}\right)\right]^{-1} \exp \left(-\alpha^{2} / \sigma^{2}\right), \quad 0<\alpha \leq r_{b} \\
& =0, \quad \alpha>r_{b} \\
\alpha & =\left[\left(R_{b}-R_{c}\right)^{2}+z_{b}^{2}\right]^{1 / 2}
\end{aligned}
$$

where $\alpha$ is the perpendicular distance from the beam centerlino and $\sigma$ is the Gaussian half-width of the current density profile.

\subsubsection{Pencil-Beam Deposition}

The normalized deposition proflle for flux surface $\rho$ of a beamlet with tangency radius $R_{b}$ and distance above the midplane $z_{b}$ is

$$
\begin{aligned}
& h^{ \pm}\left(\rho, R_{b}, z_{b}\right)=\frac{a^{2}}{2}\left[\frac{r_{\rho} \Delta^{\prime}(\rho)}{2 R_{0}}+\left(1+\frac{\Delta(\rho)}{R_{0}}\right)\right]^{-1} \frac{R}{\left(R^{2}-R_{b}^{2}\right)^{1 / 2}} \\
& \quad \times\left\{\left[r_{\rho}^{2}-\left(z_{b} / E\right)^{2}\right]^{-1 / 2} \pm \frac{\Delta^{\prime}(\rho)}{\rho}\right\}\left\{\exp \left(-D_{0}\right)+\gamma_{d} \operatorname{xp}\left[-\left(D_{0}+2 D_{1}\right)\right]\right\} \frac{1}{\lambda(\rho)}
\end{aligned}
$$

where

$$
\begin{aligned}
R & =R_{0}+\Delta(\rho) \pm\left[\rho^{2}-\left(z_{b} / E^{1}\right)^{2}\right]^{1 / 2} \\
D_{0} & =D\left(R_{1}, R_{0}+\left[a^{2}-\left(z_{b} / E\right)^{2}\right]^{1 / 2}\right) \\
D_{1} & =D\left(R_{b}, R\right)
\end{aligned}
$$

The factor $\gamma_{d}$ determines whether the second intersection of the beamlet with the flux surface is included. Thus, $\gamma_{d}=1$ when

$$
R_{b}>R_{0}-\left[a^{2}-\left(z_{b} / E\right)^{2}\right]^{1 / 2}
$$

and the beam intersects the outside wall (tangential injection), and $\gamma_{d}=0$ when

$$
R_{b}<R_{0}-\left[a^{2}-\left(z_{b} / E\right)^{2}\right]^{1 / 2}
$$

and the beam intersects the inside wall (perpendicular injection).

The beam decrement along the beamlet path between major radii $R_{1}$ and $R_{2}$ is

$$
D\left(R_{1}, R_{2}\right)=\int_{R_{1}}^{R_{2}} \frac{R^{\prime} d R^{\prime}}{\left(R^{\prime 2}-R_{b}^{2}\right)^{1 / 2}} \frac{1}{\lambda\left(\rho^{\prime}\right)}
$$

where the mean free path $\lambda$ is evaluated at the $\rho^{\prime}$ for which

$$
r_{\rho}\left(\rho^{\prime}\right)=\left\{\left[R^{\prime}-\left(R_{0}+\Delta\left(\rho^{\prime}\right)\right)\right]^{2}+\left(z_{b} / E\right)^{2}\right\}^{1 / 2}
$$


The local mean free path $(\lambda)$ for contzation of the injocted neutral is

$$
\frac{1}{\lambda(\rho)}=\frac{n_{e}\left\langle\sigma_{e} v\right\rangle_{\text {ion }}}{v_{f}}+n_{e} \sigma_{\mathrm{cx}}\left(v_{f}\right)+n_{e} \sigma_{i}\left(v_{f}\right)
$$

where $v_{f}=\left(2 E_{j, k} / m_{f}\right)^{1 / 2}$ is the fast neutral velocity. The terms give neutral ionlzation due to electron impact $\left(\sigma_{e}\right)$, hydrogenic-ion charge exchange $\left(\sigma_{\mathrm{tx}}\right)$ and impact $\left(\sigma_{i}\right)$, and impurity charge exchange and impact. The hydrogente-ion and electron cross sections are given by Riviere [35]. 'The impurity cross sectlons are taken to be $z_{k}$ thmes the hydrogenic-lon cross sections [36] where $Z_{k}$ is the charge state of impurity species $k$. Impact and charge-exchange ionization by helium lons and fast ions are also included in the above expression for $\lambda(\rho)$.

\subsection{FAST-ION THERMALIZATION}

'Thermalization of injected last lons is modeled using the moments solution of the fast-ion Fokker-Planck equation as described by Callon et al. [37].

\subsubsection{Moments of Fokker-Planck Equation}

The moments of tho fast-ion velocity distribution are given for each energy component. $j$ of each ton sonrce $k$. The fraction of the initial fast-ion energy deposited in the plasma electrons is

$$
C_{e_{j, k}}=\frac{2}{v_{j}^{2}} \int_{v(t)}^{v_{j}} v d v \frac{v^{3}}{v^{3}+v_{c}^{3}} p_{\mathrm{cx}}(v)
$$

The fraction of the initial fast-ion energy deposited in the plasma ions is

$$
C_{i, j, k}=\frac{2}{v_{f}^{2}} \int_{v(t)}^{v_{j}} v d v \frac{v_{c}^{3}}{v^{3}+v_{c}^{3}} p_{c: x}(v)
$$

'The fraction of the initial fast.ion toroidal momentum deposited in the plasma electrons is

$$
K_{t, j, k}=\frac{1}{v_{f}} \int_{v(t)}^{u_{f}} d v \frac{v^{3}}{v^{3}+v_{c}^{3}} p_{c x}(v) b(v)
$$

'The fraction of the initial fast-ion toroidal momentum deposited in all plasma ions (hydrogenic and impurities) is

$$
K_{i, k}=\frac{1}{v_{f}} \int_{v(t)}^{u_{f}} d v \frac{v_{c}^{3}}{v^{3}+v_{c}^{3}}\left(1+\frac{m_{p}}{m_{f}} \frac{Z_{\mathrm{cet \textrm {I }}}}{[Z]}\right) p_{\mathrm{cx}}(v) b(v)
$$

The fractions of the initial fast-ion toroidal momentum deposited in the hydrogenic ions $\left(\kappa_{H j, k}\right)$ and in the impurities $\left(K_{l j, k}\right)$ are

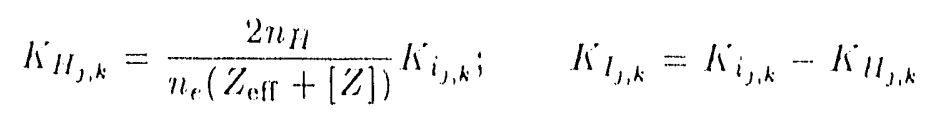

The pitch-angle scaltering term that appears in odd moments is

$$
b(v)=\left(\frac{v_{j}^{3}+v_{c}^{3} v^{3}}{v^{3}+v_{c}^{3}} \frac{v_{j}^{3}}{v_{j}}{ }^{\left[(1 / 3)\left(m_{v} / m_{j}\right)\left(z_{e+f} /[Z]\right)\right]}\right.
$$


The stacked fast-ion density moment is

$$
N_{j, k}=\int_{v(t)}^{v_{f}} d v \frac{v^{2}}{v^{3}+v_{c}^{3}} p_{c \mathbf{x}}(v)
$$

The deuterium-tritium beam-plasma fusion moment is

$$
R_{\mathrm{bp}_{,, k}}=\tau_{s} \int_{v(t)}^{v_{f}} d v \frac{v^{3}}{v^{3}+v_{c}^{3}} p_{\mathrm{cx}}(v) \sigma_{\mathrm{DT}}(v)
$$

The fast-ion perpendicular pressure moment is

$$
P_{\perp_{\jmath, k}}=\frac{2}{3 v_{f}^{2}} \int_{v(t)}^{v_{f}} v d v \frac{v^{3}}{v^{3}+v_{c}^{3}} p_{\mathrm{cx}}(v)\left[1-\frac{1}{2}\left(3 \xi_{0}^{2}-1\right) b^{3}(v)\right]
$$

The fast-ion parallel pressure moment is

$$
P_{\|_{j, k}}=\frac{2}{3 v_{f}^{2}} \int_{v^{\prime}(t)}^{v_{f}} v d v \frac{v^{3}}{v^{3}+v_{c}^{3}} p_{\mathrm{cx}}(v)\left[1+\left(3 \xi_{0}^{2}-1\right) b^{3}(v)\right]
$$

The probability that a fast ion will slow down to velocity $v$ without undergoing charge exchange on the background neutral density $n_{0}(5.1 .5)$ is

$$
\begin{aligned}
p_{\mathrm{cx}}(v) & =\left(\frac{v_{f}^{3}+v_{c}^{3}}{v^{3}+v_{c}^{3}}\right)^{-\tau_{s} / 3 \tau_{\mathrm{cx}}} \\
\tau_{\mathrm{cx}} & =\frac{1}{n_{0} v_{f} \sigma_{\mathrm{cx}}\left(v_{f}\right)}
\end{aligned}
$$

where $\sigma_{\mathrm{cx}}\left(v_{f}\right)$ is the charge-exchange cross section at the initial fast-ion velocity. The fastion velocity at time $t$ after beam turn-on is

$$
\begin{aligned}
v(t) & =\left[v_{f}^{3} e^{-3 t_{1} / \tau_{s}}-v_{c}^{3}\left(1-e^{-3 t / \tau_{s}}\right)\right]^{1 / 3}, \quad t \leq \tau_{f} \\
& =v_{T_{1}}, \quad t>\tau_{f}
\end{aligned}
$$

where $v(t)$ is used only to approximate the finite initial stacking time after initial beam turn-on. The fast-ion lifetime is

$$
\tau_{f}=\frac{\tau_{s}}{3} \ln \left[\frac{1+\left(v_{f} / v_{c}\right)^{3}}{1+\left(v_{T_{i}} / v_{c}\right)^{3}}\right]
$$

The fast-ion slowing-down time (in seconds) is

$$
\tau_{s}=0.12 \frac{\left(T_{e} / 1 \mathrm{keV}\right)^{3 / 2}}{\left(n_{e} / 10^{13} \mathrm{~cm}^{-3}\right) Z_{f}^{2}} \frac{m_{f}}{m_{p}}
$$

The initial and final fast-ion velocities are

$$
v_{f}=\left(\frac{2 E_{j, k}}{m_{f}}\right)^{1 / 2} ; \quad v_{T_{i}}=\left(\frac{2 T_{i}}{m_{f}}\right)^{1 / 2}
$$


The critical velocity and energy are

$$
v_{c}=\left(\frac{2 E_{c}}{m_{f}}\right)^{1 / 2} ; \quad E_{c}=T_{e}\left(\frac{9 \pi}{16} \frac{m_{p}}{m_{e}}\right)^{1 / 3}\left(\frac{m_{f}}{m_{p}}\right)^{1 / 3}\left(\frac{m_{f}[Z]}{m_{p}}\right)^{2 / 3}
$$

The cosine of the initial pitch angle for source $k$ is

$$
\xi_{0}=\frac{R_{c, k}}{R_{0}+r_{\rho}}
$$

\subsubsection{Complete Fokker-Planck Equation}

For most cases, the steady-state moments sulution to the fast-ion Fokker-Planck equation (7.1, 7.3.1) is sufficiently accurate and gives essentially the same plasma source profiles as the time-dependent solution described here. When the time dependence of the fast-ion velocity distribution function is important, the solution described by Fowler, Smith, and Rome [38] (referred to in this section as FSR) is used. This solution assumes that fast ions slow down on their birth flux surfaces and solves the time-dependent Fokker-Planck equation in velocity space at each radius. The model and method of solution are described in detail by FSR, and only the method of interfacing their solution with the transport model is described here.

The fast-ion source rate due to beam-energy component $j$ and injector source $k$ on flux surface $\rho$ is

$$
\begin{aligned}
\dot{n}_{f j, k}^{ \pm} & =\frac{I_{j, k}}{\epsilon V_{p}} H_{j, k}^{ \pm}(\rho) \\
\cos \theta_{j, k}^{ \pm} & =\frac{\Theta_{k} R_{c, k}}{R_{0} \pm r_{\rho}}
\end{aligned}
$$

where $I_{j, k}$ is the beam current. $V_{p}$ is the plasma volume, and $H_{j, k}^{ \pm}$is the normalized deposition profile (7.2.1) for the outer $\left(\mathrm{H}^{+}\right)$and inner $\left(\mathrm{H}^{-}\right)$intersection of the beam with the flux surface $\rho$. The corresponding initial pitch angle for each source is $\theta_{j, k}^{ \pm}$where $\Theta_{k}=1$ $(-1)$ for co-injection (counter-injection). The source term and initial pitch angle appear in Eqs. (2.1) and (2.14) of FSR, respectively.

The resulting plasma source terms are given by appropriate moments of the calculated fast-ion distribution function. The following equations from FSR define the moments of the fast-ion distribution function on the right-hand side of each equation. Equation numbers from FSR are given in parentheses.

The electron heating source is

$$
q_{\mathrm{inj}}^{e}(\rho)=-Q_{e}-P_{D}^{e}
$$

The ion heating source is

$$
q_{\text {inj }}^{i}(p)=-Q_{i}-P_{D}^{i}
$$

The fast-ion density is

$$
n_{h}(\rho)=D_{s}
$$

The source of plasma ions due to thermalized fast ions is

$$
\left.\left.s_{\mathrm{inj}}(p)=-D_{\mathrm{drag}}^{i}-D\right)_{\mathrm{drag}}^{\mathrm{i}}-D\right)_{L i}
$$


The toroidal current density perturbation (including the shielding response of plasma electrons) due to fast ions is

$$
J_{b}(\rho)=\tau_{s} Z_{f} \frac{e}{m_{f}}\left(1-Z_{f} / Z_{\text {eff }}\right)\left(-R_{\text {drag }}^{e}\right)
$$

The source of toroidal momentum for the plasma (electrons and all ions) is

$$
\dot{p}_{\phi}^{T}(\rho)=-R_{\mathrm{drag}}^{i}-R_{\mathrm{drag}}^{e}-R_{A}
$$

The fast-ion parallel $\left(p_{b \|}\right)$ and perpendicular $\left(p_{b \perp}\right)$ pressures are also calculated:

$$
\begin{aligned}
& p_{b \perp}(\rho)=E_{0} v_{0}^{3} \int x^{2} \cos ^{2} \theta f d^{3} x \\
& p_{b \|}(\rho)=2 E_{0} v_{0}^{3} \int x^{2} \sin ^{2} \theta f d^{3} x
\end{aligned}
$$

where the notation follows that in Sect. 4 of FSR. 


\section{WAVE HEATING}

\subsection{ELECTRON CYCLOTRON RESONANCE}

'Two models for electron-cyclotron resonance heating are used. The resonance layer model is appropriate for axisymmetric devices, while the nultireflection model is used for torsatron modeling.

\subsubsection{Resonance Layer Model}

A simple model for electron-cyclotron resonance heating is included by assuming that a specified amount of power $P_{\mathrm{ec}}$ at frequency $\nu_{\mathrm{ec}}$ is absorbed on a resonance surface at major radius $R_{\text {ec }}$, where

$$
R_{\mathrm{ec}}=\frac{e}{m_{\mathrm{e}} c} \frac{R_{0} B_{T}}{2 \pi \nu_{\mathrm{ec}}}
$$

The absorbed power is assumed to have a Gaussian distribution in major radius about $R_{\text {ec }}$ with a half-width $\Delta_{\text {ec }}$. Equal power is absorbed by each electron, and the deposition is therefore proportional to the local electron density. In addition, no power is absorbed inside the outermost flux surface (at $\rho=\rho_{c}$ ), where the wave frequency equals the electron plasma frequency. This occurs when the plasma electron density $n_{e}$ equals the cutoff density $n_{c}$, where

$$
n_{c}=\frac{m_{e}}{4 \pi e^{2}}\left(2 \pi \nu_{\mathrm{ec}}\right)^{2}
$$

With these assumptions, the flux-surface-integrated heating profile for $\rho \geq \rho_{c}$ is proportional to $\int(\rho)$, where

$$
f(\rho)=n_{\epsilon}(\rho) \int_{0}^{2 \pi} \exp \left[-\left(R(\rho, \theta)-R_{\mathrm{ec}}\right)^{2} / 2 \Delta_{\mathrm{ec}}^{2}\right] \sqrt{g} d \theta
$$

and $f(\rho)=0$ in the region $\rho<\rho_{c}$. The major radius $R(\rho, \theta)(6.1 .6)$ and the volume element $\sqrt{g}(6.3)$ are defined above. The normalized heating profile is

$$
h_{\mathrm{ec}}(\rho)=f(\rho) /\left(\frac{1}{V_{p}} \int_{0}^{a} f\left(\rho^{\prime}\right) V_{\rho}^{\prime}\left(\rho^{\prime}\right) d \rho^{\prime}\right)
$$

where $V_{p}$ is the total plasma volume (6.3). This normalization ensures that the entire power $P_{e c}$ is absorbed; that is, the power excluded from the resonance layer inside the cutoff region is added to the absorbed power outside this region. The resulting electron heating profile is

$$
q_{\mathrm{ec}}(\rho)=\frac{P_{\mathrm{ec}}}{V_{p}} h_{\mathrm{ec}}(\rho)
$$

\subsubsection{Multireflection Model}

The heating profile ga is approximated by a linear-alborption, multireflection model for second harmonic heating [39]. The shape of the absorption profile is assumed to be proportional to the product $f(\rho)$ of the local electron density and temperature. No power is 
absorbed inside the outermost flux surface (at $\rho=\rho_{\mathrm{c}}$ ) where the wave frequency $\nu_{\mathrm{ec}}$ equals the cutoff frequency for the extraordinary mode. This occurs for

$$
\begin{aligned}
& n_{e}=n_{\mathrm{c}} / 2 \\
& n_{\mathrm{c}}=\frac{m_{e}}{4 \pi e^{2}}\left(2 \pi \nu_{\mathrm{ec}}\right)^{2}
\end{aligned}
$$

where $n_{c}$ is the density where the wave frequency equals the plasma frequency. The factor of $1 / 2$ results from assuming that the wave frequency is twice the electron cyclotron frequency.

The normalized heating profile is

$$
\begin{aligned}
h_{\mathrm{ec}}(\rho) & = \begin{cases}f(\rho) /\left(\frac{1}{r_{p}} \int_{0}^{a} f\left(\rho^{\prime}\right) V_{\rho}^{\prime}\left(\rho^{\prime}\right) d \rho^{\prime}\right) & \rho>\rho_{\mathrm{c}} \\
0 & \rho<\rho_{\mathrm{c}}\end{cases} \\
f(\rho) & =n_{e}(\rho) T_{e}(\rho) \\
\gamma_{\mathrm{abs}} & =\frac{1}{V_{p}} \int_{\rho_{\mathrm{c}}}^{a} h_{\mathrm{ec}}\left(\rho^{\prime}\right) V_{\rho}^{\prime}\left(\rho^{\prime}\right) d \rho^{\prime}
\end{aligned}
$$

where $\gamma_{\text {ats }}$ is the fraction of the normalized deposition that is not cut off (i.e., $\gamma_{a b s}=1$ if there is no cutoff).

The fraction of the incident power absorbed by the plasma $f_{\mathrm{abs}}$ is given by

$$
\begin{aligned}
f_{\mathrm{abs}} & =F_{1}+\frac{F^{p}\left(1-F^{w}\right)\left(1-F_{1}\right)}{1-\left(1-F^{w}\right)\left(1-F^{p}\right)} \\
F_{1} & =2 \times 10^{-4}\left(n_{e 0} / 10^{12} \mathrm{~cm}^{-3}\right) T_{e 0}(\mathrm{eV}) \\
F^{p} & =6.9 \times 10^{-5} \gamma_{\mathrm{abs}}^{2}\left(n_{e 0} / 10^{12} \mathrm{~cm}^{-3}\right) T_{\mathrm{eO}}(\mathrm{eV}) \\
F^{w} & =0.02
\end{aligned}
$$

where $F_{1}$ is the fraction of the wave absorbed on the first pass through the plasma, $F^{P}$ is the fraction absorbed on subsequent passes after reflection from the wall, and $F^{w}$ is the fraction absorbed by the wall on each reflection. The fractions depend on the central $(\rho=0)$ values of the electron density $n_{e 0}$ and temperature $T_{e 0}$.

The resulting heating profile is

$$
q_{\mathrm{ec}}(\rho)=\frac{P_{\mathrm{ec}} f_{\mathrm{abs}}}{V_{p}} h_{\mathrm{ec}}(\rho)
$$

Note that the total absorbed power is less that the fraction $f_{\text {abs }}$ of the incident power when a cutoff is present $\left(\rho_{c}>0\right)$, since the power not absorbed inside the cutoff radius is not assumed to be absorbed outside the cutoff radius. 


\subsection{ION CYCLOTRON RESONANCE}

Provision is made for an arbitrary heating profile to represent ion-cyclotron heating of plasma electrons and ions. The heating profiles are given by

$$
\begin{aligned}
q_{\mathrm{ic}}^{e}(\rho) & =\frac{\int_{\mathrm{ic}}^{e} P_{\mathrm{ic}}}{V_{p}} h_{\mathrm{ic}}(\rho) \\
q_{\mathrm{ic}}^{i}(\rho) & =\frac{\int_{\mathrm{ic}}^{i} P_{\mathrm{ic}}^{i}}{V_{p}} h_{\mathrm{ic}}(\rho) \\
h_{\mathrm{ic}}(\rho) & =f(\rho) /\left(\frac{1}{V_{p}} \int_{0}^{a} f\left(\rho^{\prime}\right) V^{\prime}\left(\rho^{\prime}\right) d \rho^{\prime}\right) \\
f(\rho) & =\exp \left[-\left(\rho / \Delta_{\mathrm{ic}}\right)^{2}\right]
\end{aligned}
$$

where the total power $P_{\mathrm{i} \text {, }}$, the profile width $\Delta_{\mathrm{ic}}$, and the fraction of the power absorbed by the electrons $f_{\mathrm{ic}}^{c}$ and ions $f_{\mathrm{ic}}^{i}$ (where $f_{\mathrm{ic}}^{e}+f_{\mathrm{ic}}^{i}=1$ ) are specified arbitrarily. 


\section{FUSION}

Heating of the plasma by the thermalization of the alpha particles produced by deuteriumtritium (D-T) fusion is included in the model. The alpha particles are assumed to slow down on their birth flux surface; the thermalized particle is introduced as a source term in the helium-ion particle trarsport equation (2.1.2).

\subsection{PLASMA FAST-ALPHA HEATING}

The heating source rate of plasma electrons $\left(q_{\text {fus }}^{e}\right)$ and ions $\left(q_{\text {fus }}^{i}\right)$ due to fast-alpha slowing-down is given by

$$
\begin{aligned}
& q_{\text {fus }}^{e}(\rho)=E_{\alpha}\left(S_{\text {fus }}(\rho)+S_{\mathrm{bp}}(\rho)\right) G_{\text {fus }}^{e} \\
& q_{\text {fus }}^{i}(\rho)=E_{\alpha}\left(S_{\text {fus }}(\rho)+S_{\mathrm{bp}}(\rho)\right) G_{\text {fus }}^{i}
\end{aligned}
$$

where $E_{\alpha}$ is the initial alpha energy, $S_{\text {fus }}$ is the thermal D-T fusion rate (9.2), $S_{\text {bp }}$ is the beam-plasma D-T fusion rate (9.3), and the fractions of the fast-alpha energy deposited in electrons $\left(G_{\text {fus }}^{e}\right)$ and in ions $\left(G_{\text {fus }}^{i}\right)$ are given by

$$
\begin{aligned}
G_{\mathrm{fus}}^{e} & =\frac{2}{v_{\alpha \alpha}^{2}} \int_{0}^{v_{\alpha}} v d v \frac{v^{3}}{v^{3}+v_{c \alpha}^{3}} \\
G_{\mathrm{fue}}^{i} & =1-G_{\mathrm{fus}}^{e} \\
v_{\alpha} & =\left(2 E_{\alpha} / m_{\alpha}\right)^{1 / 2} \\
v_{\mathrm{c \alpha}} & =\left(2 E_{c \alpha \alpha} / m_{\alpha}\right)^{1 / 2} ; \quad E_{\mathrm{c \alpha}}=T_{e}\left(\frac{9 \pi}{16} \frac{m_{p}}{m_{e}}\right)^{1 / 3}\left(\frac{m_{\alpha}}{m_{p}}\right)^{1 / 3}\left(\frac{m_{\alpha}[Z]}{m_{p}}\right)^{2 / 3}
\end{aligned}
$$

where the initial alpha velocity is $v_{\alpha}$, the alpha critical energy is $E_{c \alpha}$, and the alpha particle mass is $m_{\alpha}$.

\subsection{THERMAL FUSION RATE}

The rate of thermal D-T fusion $S_{\text {fus }}^{\prime}$ is

$$
S_{\text {fus }}(\rho)=n_{H 1} n_{H 2}\langle\sigma v\rangle_{\mathrm{DT}}
$$

where $n_{H 1}$ and $n_{H 2}$ are the hydrogenic-ion species (one of which is deuterium; the other, tritium $)$ and $\langle\sigma v\rangle_{\mathrm{DT}}$ is the D-T reaction rate.

\subsection{BE.AM-PLASMA FUSION RATE}

The rate of beam-plasma D-T fusion $S_{\mathrm{bp}}$ is

$$
S_{\mathrm{bp}}(\rho)=n_{H p} \sum_{j, k} \frac{I_{j, k}}{e V_{p}} H_{j, k}(\rho) R_{\mathrm{bp}_{j, k}}(\rho)
$$

where the sum is over the injector sources $(k)$ and energy components $(j) . l_{j, k}$ is the beam current of component $j, k, V_{p}$ is the plasma volume, $H_{j, k}$ is the injected-neutral ionization 
profile (7.2), and $R_{\text {bp }}$ gives the fusion rate averaged over the slowing-rown spectrum $(7.8 .1)$. $n_{H p}$ is the density of the plasma-ion species that is not the fast-ion species. Normally, deuterium is injected into a D-T mixture, so that $n_{H p}$ is the tritlum density. 


\section{PELLET FUELING}

Fueling of the plasma by pellet injection is included either by injection of discrete pellets or with a continuous fueling source that simulates injection of many pellets closely spaced in time.

\subsection{ABLATION MODEL}

Pellets are injected in the midplane of the torus and perpendicular to the magnetic axis (inward from the outside along a major radial chord). The pellet ablation model calculates the pellet radius $r_{p}(t)$ as a function of the time $t$ since the pellet entered the plasma edge. The plasma profiles $T_{e}(\rho)$ and $n_{e}(\rho)$ are transformed to $T_{e}(t)$ and $n_{e}(t)$ with the transformations

$$
\begin{aligned}
t & =\frac{s(\rho)}{v_{p}} \\
s(\rho) & = \begin{cases}s^{+}(\rho), & t<s_{0} / v_{p} \\
s^{-}(\rho), & t>s_{0} / v_{p}\end{cases} \\
s_{0} & =s^{ \pm}(\rho=0)
\end{aligned}
$$

where $s(\rho)$ is the distance the pellet has traveled within the plasma after time $t, v_{p}$ is the pellet velocity, and $+(-)$ refers to the outer (inner) intersection of the pellet with flux surface $\rho$.

For the shifted-ellipse, axisymmetric geometry (6.5), the chord lengths are

$$
\begin{aligned}
& s^{ \pm}(\rho)=R_{0}+a+\Delta(a)-r^{ \pm}(\rho) \\
& r^{ \pm}(\rho)=R_{0} \pm r_{\rho}(\rho)+\Delta(\rho)
\end{aligned}
$$

while for the more general 3-D case, the chord lengths are determined by a chord-tracking algorithm [40].

The solution for $d r_{p}(t) / d t=f\left(T_{e}(t), n_{e}(t), \ldots\right)$ is obtained from the shielded-pellet ablation model of Milora et al. [41] using the method described by Houlberg et al. [42]. The resulting increase in plasma ion density is

$$
\begin{aligned}
\Delta n_{p}(\rho) & =\frac{2 n_{\text {pell }}}{V_{\rho}^{\prime} v_{p}} \sum_{ \pm}\left[-4 \pi r_{p}^{2}\left(t^{ \pm}\right) \frac{d r_{p}\left(t^{ \pm}\right)}{d t}\right] \frac{d_{1} \pm}{d \rho} \\
t^{ \pm} & =\frac{s^{ \pm}(\rho)}{v_{p}}
\end{aligned}
$$

where the sum is over the two intersections of the pellet with flux surface $\rho$ (at times $t^{+}$ and $\left.t^{-}\right), s^{ \pm}(\rho)$ is defined above, and $n_{\text {pell }}$ is the molecular density of the frozen pellet.

\subsection{DISCRETE PELLET FUELING}

At the time of injection of a discrete pellet, the transport evolution of the plasma is stopped, the pellet deposition profile $\left(\Delta n_{p}\right)$ is calculated $(10.1)$, the plasma densities and temperatures are incremented as shown below, and the transport evolution is restarted using the incremented plasma paraneters as initial conditions. Let the plasma parameters 
before (after) injection be unprimed (primed). The lon density of the pellet species $j$ and the plasma electron density are incremented by the pellot depositlon profile:

$$
n_{H j}^{\prime}=n_{H_{j}}+\Delta n_{p ;} \quad n_{c}^{\prime}=n_{e}+\Delta n_{p}
$$

and the plasma temperatures are decreased adiabatically,

$$
T_{i}^{\prime}=\frac{n_{T} T_{i}}{n_{T}^{\prime}} ; \quad T_{e}^{\prime \prime}=\frac{n_{e} T_{e}^{\prime}}{n_{e}^{\prime}}
$$

where $n_{T}$ is the total lon density (2.1.6).

\subsection{CONTINUOUS PELLET FUELING}

If pellets are injected frequently enough, the fueling is considered to be continuous, and the pellet ablation profile $\Delta n_{p}$ is used to calculate a normallzed fueling profile,

$$
h_{p j}(\rho)=\Delta n_{p}(\rho) /\left(\frac{1}{V_{p}} \int_{0}^{a} \Delta n_{p}\left(\rho^{\prime}\right) V_{\rho}^{\prime}\left(\rho^{\prime}\right) d \rho^{\prime}\right)
$$

The plasma fueling profile that appears in the particle transport equation of the fueled hydrogenic-ion density (2.1.1) is

$$
S_{H j}(\rho)=\frac{1}{V_{p}} S_{p j} h_{p j}(\rho)
$$

where $S_{p j}(t)$ is the rate of total particle input by pellets, which may be arbitrarily specified or controlled by feedback (11.1) to obtain a desired plasma density. 


\section{FEEDBACK CONTROL}

Feedback control is used to allow a complicated transport model to evolve to a destred state without repoated trial-and-error attempts. Feedbuck ss used ln PROC'l'R (1) to oblain a desired plasma density, (2) to obtain a desired level of fusion power in a burning plasma simulation, and (3) to match an experimontal value for the chargewexchango lon temperature. The feedback equations used in PROC'TR are all of the samo bask type. If the vartable to bo controlled is $X$ and the variable to be conllumally adjustod in tho model $t \mathrm{~s} y$, then tho foedback equation used is

$$
\frac{d y}{d t}=C\left(\frac{X-X_{0}}{\tau}+\beta \frac{d X}{d t}\right)
$$

where $X_{0}$ is the desired value of $X$. The first term on the right causes $y$ to change if $X$ is different from $X_{0}$, where the sign of the constant $C$ is chosen to onsure that $y$ changes in the currect sense to drive $X^{r}$ toward $X_{0}$. The second torm is a dampling term that prevents $X$ from oscillating around $X_{0}$. Choosing $\tau$ to be botween $5 \Delta t_{M}$ ( $\Delta t_{M}$ is the maximum timestep) and the expected rate of change of $X, \beta=2$, and $C$ to be about the expected $\delta y / \delta X$ usually is a good starting polnt for emplrically determining a stable set of feedback parameters. 'The several places in the model where a feedback equation has proved useful are described in this section.

\subsection{PLASMA DENSITY CONTROL BY GAS FEED RATE}

To obtain a clesired plasma density, the extornal gas feed rate of plasma spocies $j, S_{G i j}$, is controlled by

$$
\begin{aligned}
& \frac{d S_{G, j}}{d t}=-\frac{V_{p}}{\tau_{G}}\left(\frac{\left\langle n_{H, j}\right\rangle-n_{s j}}{\tau_{G}}+\beta_{G} \frac{d\left\langle n_{H_{j}}\right\rangle}{d t}\right) \\
& \left\langle n_{H, j}\right\rangle=\frac{1}{V_{p}} \int_{0}^{a} n_{H, j} V_{\rho}^{\prime} d \rho
\end{aligned}
$$

where $V_{p}$ is the plasma volume, $n_{s j}$ is the desired volume-average density for species $j$, $\left\langle n_{H j}\right\rangle$ is the model value of the volume-average density, $\beta_{G}=2$, and $\tau_{G}$ is usually chosen to be $5 \Delta t_{M}$ (where $\Delta t_{M}$ is the maximum timestep). Separate control of the density of each plasma lon spectes is especially desirable in burning-plasma simulations. A similar equalion is used to obtaln a desirod line-average electron density in a nonburning plasma. The fueling rate that results from this feedback equation may be used either as a gas rato $S_{G j}(5.1 .1)$ or as an equivalent continuous pellet fueling rate $S_{j j}\left(10 . y^{\prime}\right)$.

\subsection{LINE DENSITY CONTROL BY PARTICLE DIFFUSIVITY}

In simulating an actual plasma shot, the moasured gas foed rate and the complote wallrocycle model are used. Therefore, the level of plasma diffusivity is continuously andjusted to obtain the measured time-dependent line density. The foedback eguation for the diffusivity multiplier $C_{D}(t)$ is

$$
\frac{\left.d C_{D}\right)}{d t}=\frac{\left.\partial C_{l}^{\prime}\right)}{\partial n}\left(\frac{n_{e}-n_{e}^{s}}{\left.\tau_{l}\right)}+\beta_{l} \frac{d\left(n_{c}-n_{e}^{s}\right)}{d t}\right)
$$

where $n_{e}^{g}(t)$ is the measured line-average density and $n_{c}(t)$ is the modoled line-average density. Cood results are obtained by choosing $r_{l}$ ) $=5 \Delta t_{M}$ (where $\Delta t_{M}$ is the maximum 
(1) mestep), $\beta_{D}=2$, and the constint $O C_{D} / \partial n$ equal to the ratio of a chango in diflualvity to the resulting change in line density. The resulting $C_{p(t)}(t)$ shows the requiled temporal dopendence for a roullatle model of diffusivity. 'losting of prodletive modole for plasma particle transport is reduced by this feodbeck oquation to finding a model for particle difluslvity that glves $C D(t) \simeq$ constant.

\subsection{LINE DENSITY CONTROL BY LIMITER RECYCLING}

It is somethes Interesting to oxamine the thme dependence of tho limiter rocycling conffictent. $R_{L}(5,1,1)$ necessary for a given particle transport modol to reproduce tho measured linendensity evolutlon durltge a given plasma shot. The feodback ocpuation for this case is

$$
\frac{d R_{L}}{d t}=-\frac{\partial R_{L}}{\partial n}\left(\frac{\ddot{n}_{e}-\ddot{n}_{e}^{s}}{\tau_{L}}+\beta_{L} \frac{d\left(\ddot{n}_{e}-\ddot{n}_{e}^{s}\right)}{d l}\right)
$$

where $n_{e}$ and $n_{e}^{s}$ are the modolod and measured line-average electron donstlies and the constants aro chosen as described above.

\subsection{FUSION POWER CONTROL BY VARIABLE DENSITY}

The fusion power produced by a burning plasma simulation may be controlled by changIng the fuel donsity, This control Involves the use of two foedback equations. The desired plasma density of species $j, n_{a, j}$, is governed by

$$
\begin{aligned}
& \frac{d n_{s, j}}{d l}=-\frac{\partial n}{d P}\left(\frac{P_{\text {fut }}-P_{\text {sel }}}{\tau_{d}}+\rho_{d} \frac{d P_{\text {fus }}}{d l}\right) \\
& P_{\text {fus }}=E_{n}^{\prime} \int_{0}^{a}\left(S_{\text {fut }}^{\prime}(\rho)+S_{b p}(\rho)\right) V_{\rho}^{\prime} d \rho
\end{aligned}
$$

where Prus is the modeled fuston power, which is the volume integral of the sum of the

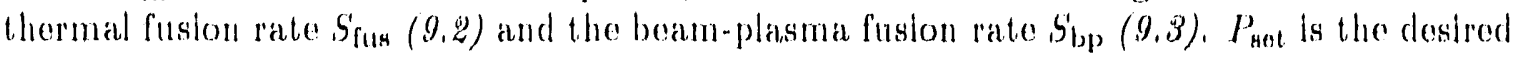
fuston power, assuming an energy $E_{n}$ per fuston event. The rosulting timo-dependent $n_{g j}$ is used as the destred valuo for the fuoling rate foedback oguation $(11,1)$.

\subsection{FUSION POWER CONTROL BY TOROIDAL, FIELD RIPPLE}

The fusion power may also be controlled by changing the plasma temperature at a flxed plasma density. One way to change the temperalure is to control the bon heat conductivity by lmposing a time-variable toroddal magnotic fleld ripple. The feedback eguation for the odge ripple o o ls

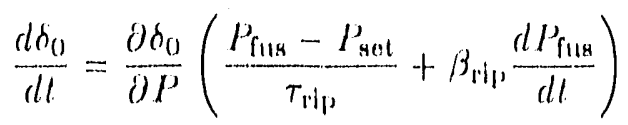

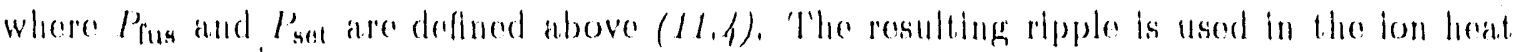

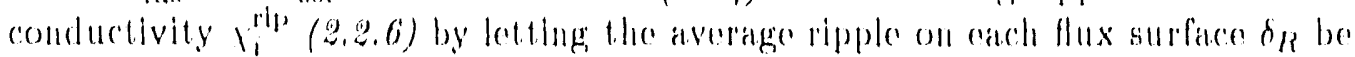

$$
h_{h}(l, 1)=\delta_{01}(t) \int_{k}(l)
$$

where $\int_{k}$ is the spatial profile of the ripple. 


\section{$11 . \theta$ ION TEMPERATURE CONTROL BY ION CONDUCTIVITY}

The simulated charge-exchange lon temperature $T_{i}^{\mathrm{cx}}(12.1 .9)$ may be made to equal a given value $T_{i}^{n p p}$ by adjusting the level of lon hoat conduction with the feodback oquation

$$
\frac{d C_{x}}{d t}=\frac{\partial C_{x}^{\prime}}{\partial T_{i}}\left(\frac{T_{i}^{\mathrm{dx}}-T_{i}^{\text {app }}}{\tau_{x}}\right)
$$

where $C_{\chi}$ lo the $\chi_{i}$ multiplier and $\tau_{\chi}$ is the feedback response time. The feedback techndque works best for $C_{\chi}$ if the damping factor $\beta_{\chi}=0$. 


\section{DIAGNOSTICS}

\subsection{DIAGNOSTIC VALUES FOR EXPERIMENTAL COMPARISON}

For comparison of the model with experimental data, plasma parameters measured by experimental dlagnostics are calculated. The defintions of common parameters used for comparison between the modeled and measured plasmas are given in this section. Model parameters are deftned in the sections given in the index.

\subsubsection{Line-Average Density}

For shifted-ellipse, axisymmetric geometry (6.5), the line-average electron density measured along a vertical chord at major radius $R_{F}$ is

$$
\begin{aligned}
\bar{n}_{e}^{\mathrm{FIH}} & =\frac{2}{L_{c}} \int_{0}^{a} d \rho n_{e}(\rho) \frac{d z}{d \rho} \\
z(\rho) & =E\left[r_{\rho}^{2}-\left(R_{F}-R_{0}+\Delta(\rho)\right)^{2}\right]^{1 / 2}
\end{aligned}
$$

where $L_{c}$ is the total chord length used to define $\ddot{n}_{e}$ experimentally.

The horizontal line-average electron density measured in the plasma midplane is

$$
\bar{n}_{e}=\frac{2}{L_{\mathrm{c}}} \int_{0}^{a} n_{e}(\rho) r_{\rho}^{\prime} d \rho
$$

For the more general 3-D gcometry or for an arbitrary chord in the axisymmetric case, the line density for any chord is

$$
\bar{n}_{e}=\frac{1}{L_{c}} \int n_{e} d s
$$

where the chord mapping $s(\rho)$ is derived with a chord-tracking algorithm [40].

\subsubsection{Loop Voltage}

The loop voltage is defined as the average between the inner and the outer voltage drops around the plasma. Thus,

$$
V_{L}=2 \pi R_{0} E_{z}(\rho=a)
$$

where $E_{z}(\rho=a)(2.1 .7)$ is the average toroidal electric field at the plasma edge.

\subsubsection{Beta}

'The average pressure, including the fast-ion component, is given by

$$
\ddot{p}_{j}=\frac{1}{V_{s}} \int_{0}^{a_{s}} p_{j}(\rho) V_{p}^{\prime} d \rho
$$

where $V_{s}$ is the plasma volume enclosed within the confined plasma region excluding the scrape-off,

$$
V_{s}=\int_{0}^{u_{s}} V_{p}^{\prime} d p
$$


The parallel $(\mu=\|)$ and perpendicular $(\mu=\perp)$ pressures are

$$
p_{\mu}(\rho)=n_{e} T_{e}+n_{T} T_{i}+p_{b_{\mu}}
$$

where the fast-ion pressures $p_{b \mu}$ are defined above (7.1).

Toroidal $\left(\beta_{T}\right)$ and poloidal $\left(\beta_{I}\right)$ betas are defined by

$$
\begin{aligned}
& \beta_{T}=\frac{\left(\bar{p}_{\|}+\bar{p}_{\perp}\right) / 2}{B_{T}^{2} / 2 \mu_{0}} \\
& \beta_{I}=\frac{E^{2}+1}{2} \frac{\left(\bar{p}_{\|}+\bar{p}_{\perp}\right) / 2}{\tilde{B}_{p}^{2} / 2 \mu_{0}}
\end{aligned}
$$

where $\beta_{I}$ is often referred to as the "engineering beta" and

$$
\ddot{B}_{\mathfrak{p}}=\frac{\mu_{0} I_{T}}{2 \pi r_{s}}
$$

Perpendicular beta as measured by a diamagnetic loop is

$$
\beta_{\perp}=\frac{E^{2}+1}{2} \frac{\bar{p}_{\perp}}{\tilde{B}_{p}^{2} / 2 \iota_{0}}
$$

The actual value of poloidal beta ("MHD beta") is

$$
\begin{aligned}
\beta_{J} & =\frac{\left(\bar{p}_{\|}+\bar{p}_{\perp}\right) / 2}{B_{\theta}^{2} / 2 \mu_{0}} \\
B_{\theta}^{2} & \left.=\left(\mu_{0} I_{T} / 2 \pi\right)^{2}\left(\langle\sqrt{g}\rangle_{\theta} i g_{\theta \theta} / \sqrt{g}\right\rangle_{\theta}\right)^{-1}
\end{aligned}
$$

where the metrics $g$ are defined above $(6.5)$.

\subsubsection{Energy Confinement Time}

The energy confinement time that is usually determined experimentally ("gross confinement time") is the ratio of the stored energy (determined magnetically) to the total power incident on the plasma. This time is

$$
\begin{aligned}
\tau_{E}^{*} & =\frac{W}{P_{\mathrm{oh}}+P_{\mathrm{inj}}-\dot{W}} \\
W & =\frac{3}{4}\left(\bar{p}_{\|}+\ddot{p}_{\perp}\right) \\
P_{\mathrm{oh}} & =\int_{0}^{u_{s}} E_{z} J_{z} V_{\rho}^{\prime} d \rho \\
P_{\mathrm{inj}} & =\frac{1}{\epsilon} \sum_{j, k} I_{j, k} E_{j, k}
\end{aligned}
$$

where $W$ is the stored energy excluding the scrape-off, $P_{\text {oh }}$ is ohmic heating power corrected for inductance, and $P_{\text {inj }}$ is the totai neutral beam heating power delivered to the torus. 


\subsubsection{Internal Inductance}

The pløsma internal inductance is

$$
\ell_{i}=\frac{2 \mu_{0}}{B_{\theta}^{2}\left(a_{s}\right)} \frac{1}{V_{s}} \int_{0}^{a s}\left(B_{\theta}^{2}(\rho) / 2 \mu_{0}\right) V_{\rho}^{\prime} d \rho
$$

where $B_{\theta}^{2}$ and $V_{s}$ are defined above (12.1.3).

\subsubsection{Safety Factor}

A simplified definition of the safety factor ("engineering $q$ ") is

$$
q_{I}=\frac{E^{2}+1}{2} \frac{r_{s} B_{T}}{R_{0} \tilde{B}_{p}}
$$

where $\tilde{B}_{p}$ is defined above (12.1.3).

The complete safety factor is used in the transport model; thus

$$
q_{\psi}=q\left(\rho=a_{s}\right)
$$

where $q(\rho)$ is defined above (2.2.1).

\subsubsection{Neutron Rate}

The thermal neutron rate for a deuterium plasma is used to infer the ion temperature. The total emission rate of neutrons from the plasma is

$$
R_{\mathrm{DD}}=\frac{1}{4} \int_{0}^{a} n_{\mathrm{D}}^{2}\langle\sigma v\rangle_{\mathrm{DD}} V_{\rho}^{\prime} d \rho
$$

where $n_{\mathrm{D}}$ is the density of deuterons in the plasma and $\langle\sigma v\rangle_{\mathrm{DD}}$ is the total reaction rate with the branching ratio assumed to be $1 / 2$.

\subsubsection{Radiated Power}

The total power radiated by impurities in the plasma is

$$
P_{\mathrm{rad}}=\int_{0}^{a} q_{\mathrm{rad}}(\rho) V_{\rho}^{\prime} d \rho
$$

For comparison with bolometer measurements, the charge-exchange and radiation losses from hydrogenic neutrals $(5.1 .4)$ must also be considered.

\subsubsection{Charge-Exchange Ion Temperature}

The calculation of an apparent ion temperature from a measured charge-exchange spectrum is described by Davis, Mueller, and Keane [43]. The charge-exchange flux at energy 
$E$ for hydrogenic-ion species $j$ is

$$
\begin{aligned}
\Gamma_{\mathrm{cx}}(E) & =\int_{0}^{s_{\mathrm{m}}} d s\langle\sigma v\rangle_{\mathrm{cx}} n_{0} n_{H j} g_{+} e^{-D(s)} \\
\langle\sigma v\rangle_{\mathrm{cx}} & =\sigma_{\mathrm{cx}}\left(v_{E}\right) v_{E}, \quad v_{E}=\left(2 E / m_{H j}\right)^{1 / 2} \\
g_{+}(E) & =\frac{2}{\pi} \frac{E^{1 / 2}}{T_{i}^{3 / 2}} e^{-E / T_{i}} \\
D(s) & =\int_{0}^{s} \frac{d s^{\prime}}{\lambda\left(s^{\prime}\right)} \\
\frac{1}{\lambda} & =\frac{n_{e}\langle\sigma v\rangle_{e}}{v_{E}}+n_{T}\left(\sigma_{\mathrm{cx}}\left(v_{E}\right)+\sigma_{i}\left(v_{E}\right)\right)
\end{aligned}
$$

where the values of the plasma parameters on surface $\rho$ at a distance $s(\rho)$ along the viewing chord are calculated from the magnetic geometry with a chord-tracking algorithm [40].

The apparent ion temperature $T_{i}^{c x}$ is given by a least-squares fit of the function

$$
y(E)=y_{0}-E / T_{i}^{\mathrm{cx}}
$$

to the calculated values

$$
y_{i}=\ln \left(E_{i}^{-1 / 2} \Gamma_{\mathbf{c x}}\left(E_{i}\right)\right)
$$

where the spectrum is measured at the energies $E_{i}$ for $i=1, \ldots, n$. Thus, $T_{i}^{\mathrm{cx}}$ is given by

$$
T_{i}^{\mathrm{cx}}=\left(\frac{1}{n}\left(\sum_{i} E_{i}\right)^{2}-\sum_{i} E_{i}^{2}\right) /\left(\sum_{i} y_{i} E_{i}-\frac{1}{n} \sum_{i} E_{i} \sum_{i} y_{i}\right)
$$

\subsubsection{Spectroscopic Lines}

The line emission $S_{\text {obs }}^{\ell}$ observed by a spectrometer for a line emitted by charge state $q_{\ell}$ of impurity species $j$ is given by

$$
S_{\mathrm{obs}}^{\ell}=\int_{0}^{s_{\mathrm{m}}} d s n_{e} n_{I j}^{q \ell} b_{r}^{\ell}\langle\sigma v\rangle_{\mathrm{ex}}^{\ell}
$$

where the values of the plasma parameters on surface $\rho$ at a distance $s(\rho)$ along the viewing chord are calculated from the magnetic geometry with a chord-tracking algorithm [40]. $b_{\tau}^{\ell}$ is the branching ratio and $\langle\sigma v\rangle_{e x}^{\ell}$ is the excitation rate for the observed line.

\subsection{INTEGRATED BALANCES}

The volume integrals of the terms in the transport equations are computed for printing and plotting and for use in estimating confinement time and transport coefficients. In this section, the notation used for these balances is detailed and the derived confinement parameters are defined. The neoclassical fluxes appropriate for a nonaxisymmetric plasma, which are used for comparison with the experimentally inferred fluxes, are also given.

If $f(\rho)$ represents a term in a transport equation, then the corresponding volumeintegrated value $F(\rho)$ is

$$
F(\rho)=C_{u} \int_{0}^{\rho} f\left(\rho^{\prime}\right) V_{\rho}^{\prime}\left(\rho^{\prime}\right) d \rho^{\prime}
$$


where $C_{u}$ is a constant which properly converts the units.

The units used in the transport model are centimeters (distance), milliseconds (time), electron volts (energy and temperature), and amperes (current).

The integrated hydrogenic ion density equation (2.1.1) is in number per second and $C_{u}=10^{3} \mathrm{~ms} / \mathrm{s}$. The terms for species $j$, in the order in which they appear in (2.1:1), are neutral ionization minus fusion loss $\dot{N}_{\text {ion }}^{j}$, diffusion $\dot{N}_{\text {conv }}^{j}$, convective flow $\dot{N}_{\text {pnch }}^{j}$, fueling due to thermalized fast ions $\dot{N}_{\text {beam }}^{j}$, and parallel loss in the scrape-off layer $\dot{N}_{\text {sol }}^{j}$.

When the ion density equation is not solved, the definition of $\Gamma_{H j}$ given at the end of Section (2.1.1) applies and

$$
\begin{aligned}
& \dot{N}_{\text {conv }}^{j}=-\left(\dot{N}_{\text {ion }}^{j}+\dot{N}_{\text {beam }}^{j}-\dot{N}_{\text {sol }}^{j}\right) \\
& \dot{N}_{\text {pnch }}^{j}=0
\end{aligned}
$$

The integrated electron temperature equation (2.1.5) gives the electron power balance in kilowatts, and $C_{t}=1.6 \times 10^{-19}$ joule/eV. The terms, in the order in which they appear in (2.1.5), are neutral beam heating of electrons $P_{\text {inj }}^{e}$, heat conduction $p_{\text {cond }}$, convection $P_{\text {conv }}^{e}$, electron-ion thermalization $P_{\text {ei }}^{e}$, impurity radiation $P_{\text {rad }}^{e}$, neutral ionization $P_{\text {ion }}^{e}$, ohmic heating $P_{\mathrm{oh}}^{e}$, wave heating $P_{\mathrm{ec}}^{e}$, fusion heating $P_{\mathrm{fus}}^{e}$, and parallel loss in the scrape-off layer $P_{\mathrm{sol}}^{e}$.

When the $T_{e}$ equation is not solved, the difference between the volume heating sources and volume plus convective losses is attributed to heat conduction; thus

$$
P_{\mathrm{cond}}^{e}=P_{\mathrm{inj}}^{e}+P_{\mathrm{oh}}^{e}+P_{\mathrm{ec}}^{e}+P_{\mathrm{fus}}^{e}-P_{\mathrm{conv}}^{e}-P_{\mathrm{ei}}^{e}-P_{\mathrm{rad}}^{e}-P_{\mathrm{ion}}^{e}-P_{\mathrm{sol}}^{e}
$$

The integrated ion temperature equation (2.1.6) gives the ion power balance in kilowatts and $C_{u}=1.6 \times 10^{-19}$ joule/eV. The terms, in the order in which they appear in (2.1.6), are ion-electron thermalization $P_{\text {ei }}^{i}$, heat conduction $P_{\text {cond }}^{i}$, convection $P_{\text {conv }}^{i}$, thermal chargeexchange loss including reionization $P_{\mathrm{cx}}^{i}$, neutral-beam fon heating $P_{\mathrm{inj}}^{i}$, fusion heating $P_{\text {fus }}^{i}$, and parallel loss in the scrape-off layer $P_{\text {sol }}^{i}$.

When the $T_{i}$ equation is not solved, the difference between the volume heating sources and volume plus convective losses is attributed to heat conduction; thus

$$
P_{\text {cond }}^{i}=P_{\mathrm{ei}}^{i}+P_{\mathrm{inj}}^{i}+P_{\mathrm{fus}}^{i}-P_{\mathrm{conv}}^{i}-P_{\mathrm{c} \mathrm{x}}^{i}-P_{\mathrm{sol}}^{i}
$$

The energy confinement times for the plasma inside flux surface $\rho$ are

$$
\begin{aligned}
\tau_{E}(\rho) & =\frac{W_{e}(\rho)+W_{i}(\rho)}{P_{\mathrm{h}}^{e}+P_{\mathrm{inj}}^{i}-\dot{W}_{e}-\dot{W}_{i}} \\
\tau_{E e}(\rho) & =\frac{W_{e}(\rho)}{P_{\mathrm{h}}^{e}-P_{\mathrm{ei}}^{e}-\dot{W}_{e}} \\
\tau_{E i}(\rho) & =\frac{W_{i}(\rho)}{P_{\mathrm{ei}}^{i}+P_{\mathrm{inj}}^{i}-\dot{W}_{i}}
\end{aligned}
$$

where the stored energies are

$$
\begin{aligned}
& W_{c}(\rho)=\frac{3}{2} \int_{0}^{\rho} n_{c}\left(\rho^{\prime}\right) T_{e}\left(\rho^{\prime}\right) V_{\rho}^{\prime}\left(\rho^{\prime}\right) d \rho^{\prime} \\
& W_{i}(\rho)=\frac{3}{2} \int_{0}^{\rho}\left(\sum_{j} n_{H_{l}\left(\rho^{\prime}\right)}+\sum_{j} n_{I_{j}}\left(\rho^{\prime}\right)\right) T_{i}\left(\rho^{\prime}\right) V_{\rho}^{\prime \prime}\left(\rho^{\prime}\right) d \rho^{\prime}
\end{aligned}
$$


the total electron heating power is

$$
P_{\mathrm{h}}^{e}=P_{\mathrm{inj}}^{e}+P_{\mathrm{oh}}^{e}+P_{\mathrm{ec}}^{e}
$$

and the $\dot{W}$ terms are given by the volume integrals of the sums of the $\partial n / \partial t$ and $\partial T / \partial t$ terms in the $T_{e}(2.1 .5)$ and $T_{i}(2.1 .6)$ equations. The $\dot{W}$ terms are zero in profile analysis mode.

The total particle confinement time for flux surface $\rho$ is defined by

$$
\begin{aligned}
\tau_{p}(\rho) & =\frac{\sum_{j} N_{H j}}{\sum_{j}\left(\dot{N}_{\text {ion }}^{j}+\dot{N}_{\text {bearn }}^{j}+\dot{N}_{H j}\right)} \\
N_{H j}(\rho) & =\int_{0}^{\rho} n_{H j}\left(\rho^{\prime}\right) V_{\rho}^{\prime}\left(\rho^{\prime}\right) d \rho^{\prime}
\end{aligned}
$$

where $N_{H j}(\rho)$ is the total number of ion of species $j$ contained within surface $\rho$ and $\dot{N}_{H j}$ is the volume integral of the $\partial n_{H j} / \partial t$ term in the hydrogenic-ion density equation (2.1.1).

The total rates of collisional transport of species $a$ across flux surface $\rho$ of particles $\dot{N}_{a}^{n c}$ and heat $P_{a}^{n c}$ are given by

$$
\begin{aligned}
& \dot{N}_{a}^{n c}=V_{\rho}^{\prime}\left\langle(\nabla \rho)^{2}\right\rangle_{\psi}\left(\Gamma_{a}^{s}+\Gamma_{a}^{n a}\right) \\
& P_{a}^{n c}=V_{\rho}^{\prime}\left\langle(\nabla \rho)^{2}\right\rangle_{\psi}\left(q_{a}^{s}+q_{a}^{n a}\right)
\end{aligned}
$$

where the nonaxisymmetric helical ripple transport fluxes $\left(\Gamma_{a}^{n a}\right.$ and $q_{a}^{\text {na }}$ ) are given above (2.2.7). The axisymmetric neoclassical transport is given approximately by

$$
\begin{aligned}
\Gamma_{a}^{s} & =-D_{a}^{s} \frac{\partial n_{a}}{\partial \rho} \\
q_{a}^{s} & =-n_{a} \chi_{a}^{s} \frac{\partial T_{a}}{\partial \rho}
\end{aligned}
$$

The transport coefficients $D_{\mathfrak{a}}^{s}, \chi_{\mathfrak{a}}^{s}$ are described by Hinton and Hazeltine [4].

The $T_{e}$ profile may be used to infer an electron heat conduction coefficient $\chi_{e}^{\text {inv }}(\rho)$ with the assumption that the entire electron heat conduction flux is proportional to only the electron temperature gradient. The coefficient inierred from this profile inversion is

$$
\chi_{e}^{\text {inv }}(\rho)=\frac{P_{\text {cond }}^{e}(\rho)}{-V_{\rho}^{\prime \prime}\left\langle(\nabla \rho)^{2}\right\rangle_{\psi} n_{e}\left(\partial T_{e} / \partial \rho\right)}
$$

where $P_{\text {cond }}^{e}(\rho)$ is the power flow across surface $\rho$ due to electron heat conduction as defined above. Note that, while this inversion is used primarily when $T_{e}(\rho)$ is the (constant) measured value, the inversion may also be performed when the $T_{e}$ equation is solved. 


\section{REFERENCES}

[1] S. I. Braginskii, "Transport Processes in a Plasma," in Reviews of Plasma Physics, vol. 1, pp. 205-309, Consultants Bureau, New York, 1965.

[2] J. Blum and J. L. Foll, "Plasma Equilibrium Evolution at the Resistive Diffusion Timescale," Comput. Phys. Rep. 1, 465 (1984).

[3] D. E. Post et al., "Steady-State Radiative Cooling Rates for Low Density, High Temperature Plasmas," At. Data Nucl. Data Tables 20, 397 (1977).

[4] F. L. Hinton and R. D. Hazeltine, "Theory of Plasma Transport in Toroidal Confinement Systems," Rev. Mod. Phys. 48, 239 (1976).

[5] S. P. Hirshman and D. J. Sigmar, "Neoclassical Transport of Impurities in Tokamak Plasmas," Nucl. Fusion 21, 1079 (1981).

[6] K. T. Tsang and E. A. Frieman, "Toroidal Plasma Rotation in Axisymmetric and Slightly Non-Axisymmetric Systems," Phys. Fluids 19, 747 (1976).

[7] J. T. Hogan, "Collisional Transport of Momentum in Axisymmetric Configurations," Phys. Fluids 27, 2308 (1984).

[8] B. A. Carreras et al., "Transport Effects Induced by Resistive Ballooning Modes and Comparison with High- $\beta_{p}$, ISX-B Tokamak Confinement," Phys. Rev. Lett. 50, 503 (1983).

[9] P. H. Diamond et al., "Kinetic Theory of Resistive Ballooning Modes," Phys. Fluids 28, $1116(1985)$.

[10] C. S. Chang and F. L. Hinton, "Effect of Finite Aspect Ratio on the Neoclassical Ion Thermal Conductivity in the Banana Regime," Phys. Fluids 25, 1493 (1982).

[11] N. A. Uckan, K. T. Tsang, and J. D. Callen, "Toroidal Field Ripple Effects in Large Tokamaks," in Proceedings of the 6th Symposium on Engineering Problems of Fusion Research, pp. 1105-1109, Institute of Electrical Electronics Engineers, New York, 1976.

[12] D. E. Hastings, W. A. Houlberg, and K. C. Shaing, "The Ambipolar Electric Field in Stellarators," Nucl. Fusion 25, 445 (1985).

[13] S. P. Hirshman, R. J. Hawryluk, and B. Birge, "Neoclassical Conductivity of a Tokamak Plasma," Nucl. Fusion 17, 611 (1977).

[14] K. Audenaerde, G. Emmert, and M. Gordinier, "SPUDNUT': A Transport Code for" Neutral Atoms in Plasma," J. Comput. Phys. 34, 268 (1980).

[15] H. C. Howe, "Dependence of Hydrogen Recycling on Molecular Effects in Tokamaks," J. Nucl. Mater. 111 \& 112, 424 (1982). 
[16] R. L. Freeman and E. M. Jones, Atomic Collision Processes in Plasma Physics Experiments: Analytic Expressions for Selected Cross-Sections and Maxwellian Rate Coefficierts, Technical Report CLM-R 137, Culham Laboratory, Abingdon, England, 1974.

[17] E. M. Jones, Atomic Collision Processes in Plasma Physics Experiments: Analytic Expressions for Selected Cross-Sections and Maxwellian Rate Coefficients II, Technical Report CLM-R 175, Culham Laboratory, Abingdon, England, 1977.

[18] O. S. Oen and M. T. Bobinson, "Computer Studies of the Reflection of Light Ions from Solids," Nucl. Instrum. Methods 132, 647 (1976).

[19] W. Eckstein and H. Verbeek, "Reflection of H, D, and He from C, Ti, Ni, Mo, W, and Au," J. Nucl. Mater. 76 \& 77, 365 (1978).

[20] D. Smith; "Analytical Expressions: Physical Sputtering," in Proc. of the Workshop on Sputtering, Report CONF-790775, Argonne Natl. Lab., Argonne, Ill, 1980.

[21] O. S. Oen and M. T. Robinson, "Computer Studies of the Penetration Depth of LowEnergy Light Ions in Solids," in Applications of Ion Beams to Materials, pp. 329--333, Institute of Physics, London, 1976.

[22] H. C. Howe, "Hydrogen Recycle Modeling and Measurements in Tokamaks," J. Nucl. Mater. 93 \& 94, 17 (1980).

[23] K. Wilson and M. Baskes, "Deuterium Trapping in Irradiated 316 Stainless Steel," J. Nucl. Mater, 76 \& 77, 291 (1978).

[24] P. Wienhold et al., "Numerical Evaluation of Hydrogen Release from and Volume Distribution in First Wall Materials," J. Nucl. Mater. 85 \& 86, 1001 (1979).

[25] H. C. Howe and R. A. Langley, "Measurement of the Recombination Rate Coefficient of a Tokamak Vacuum Vessel Wall," J. Vac. Sci. Technol. A 1, 1435 (1983).

[26] G. A. Emmert et al., "Electric Sheath and Presheath in a Collisionless, Finite Ion Temperature Plasma," Phys. Fluids 23, 803 (1980).

[27] C. M. Strawitch and G. A. Emmert, "Non-Ambipolar Transport in a Magnetic Divertor," Nucl. Fusion 21, 1291 (1981).

[28] H. C. Howe, Physics Models in the Tolamak Transport Code PROCTR, Technical Report OR.NL/TM-9537, Martin Marietta Energy Systems, Inc., Oak Ridge Natl. Lab., 1985.

[29] D. K. Lee, "Magnetic Field, Vector Potential and Their Derivatives due to Currents in Closed Polygons of Wire," Comput. Phys. Commun. 25, 181 (1982).

[30] S. P. Hirshman and D. K. Lee, "MOMCON: a Spectral Code for Obtaining ThreeDimensional Magnetohydrodynamic Equilibria," Comput. Phys. Commun. 39, 161 (1936). 
[31] S. P. Hirshman and J, C. Whitson, "Steepest-Descent Moment Method for ThreeDimensional Magnetohydrodynamic Equilibria," Phys. Fluids 26, 3553 (1983).

[32] L. L. Lao, S. P. Hirshman, and R. M. Wieland, "Variational Moment Solution to the Grad-Shafranov Equation," Phys. Fluids 24, 1431 (1981).

[33] K. McGuire and D. C. Robinson, "Sawtooth Oscillations in a Small Tokamak," Nucl. Fusion 19, 505 (1979).

[34] J. A. Rome, J. D. Callen, and J. F. Clarke, "Neutral-Beam Injection into a Tokamak: Fast-Ion Spatial Distribution for Tangential Injection," Nucl. Fusion 14, 141 (1974).

[35] A. C. Riviere, "Penetration of Fast Hydrogen Atoms into a Fusion Reactor Plasma," Nucl. Fision 11, 363 (1971).

[36] R. E. Olson et al., "Charge-State Dependence of Electron Loss from H by Collisions with Heavy, Highly Stripped lons," Phys. Rev. Lett. 41, 163 (1978).

[37] J. D. Callen et al., "Neutral Beam Injection into Tokamaks," in Plasma Physics and Controlled Nuclear Fusion Research 1974: Proceedings of the 5th International Conference, pp. 645-658, International Atomic Energy Agency, Vienna, 1975.

[38] R. H. Fowler, J. Smith, and J. A. Rome, "FIFPC -- A Fast Ion Fokker-Planck Code," Comput. Phys. Commun. 13, 323 (1978).

[39] R. C. Goldfinger and D. B. Batchelor, "Second Harmonic Electron Cyclotron Heating Calculations for the ATF Torsatron," in Applications of Radio-Frequency Power to Plasmas, AIP Conf. Proc. No. 159, pp. 65-68, American Institute of Physics, New York, 1987.

[40] S. E. Attenberger, W. A. Houlberg, and S. P. Hirshman, "Some Practical Considerations Involving Spectral Representations of 3-D Plasma Equilibria," J. Comput. Phys. 72, 435 (1987).

[41] S. L. Milora and C. A. Foster, "A Revised Neutral Gas Shielding Model for PelletPlasma Interactions," IEEE Trans. Plasma S'ri. PS-6, 578 (1978).

[42] W. A. Houlberg et al., PELLE'T A Computer Routine for Modeling Pellet Fueling in Tokamak Plasmas, Technical Report ORNL/TM-6549, Union Carbide Corp. Nuclear Div., Oak Ridge Natl. Lab., 1979.

[43] S. L. Davis, D. Mueller, and C. J. Keane, "Mass Resolved Charge-Exchange System on the Poloidal Divertor Experiment," Rev. Sei. Instrum. 54, 315 (1983). 


\section{INDEX OF VARIABLES}

The number after each variable is the sectlon in the text where the model for or deflnition of the variable is given.

\begin{tabular}{|c|c|c|c|c|c|}
\hline$a$ & 6.1 .5 & $J_{b}$ & 7.1 & $q_{\text {fion }}$ & 5.1 .4 \\
\hline$a_{d}$ & 5.1 .1 & $J_{\mathrm{oh}}$ & 2.1 .7 & qoh & 2.1 .7 \\
\hline$a_{s}$ & 6.1 .5 & $J_{Z}$ & 2.1 .7 & $q_{\text {rad }}$ & 2.2 .9 \\
\hline$A_{u \prime}$ & 5.1 .2 & $K_{j}$ & 2.2 .3 & $r_{p}$ & 6.1 .6 \\
\hline$B_{T}$ & 2.2 .1 & $k_{r}$ & 4.3 & $r_{s}$ & 0.1 .6 \\
\hline$B_{\theta}$ & 2.1 .7 & $m_{e}$ & 2.2 .1 & $r_{a}$ & 6.1 .6 \\
\hline$C_{D}$ & 11.2 & $m_{H j}$ & 2.2 .1 & $R$ & 6.1 \\
\hline$c_{j}$ & 4.1 & $m_{l j}$ & 2.2 .1 & $R_{L}$ & 5.1 .1 \\
\hline$C^{\prime} T j$ & 4.1 & $m_{p}$ & 2.2 .1 & $R_{0}$ & 6.1 .1 \\
\hline$c_{\theta}$ & 6.4 & $n_{b}$ & 7.1 & $R_{w}$ & 5.1 .1 \\
\hline$c_{\phi}$ & 6.4 & $n_{e}$ & 2.2 .1 & $\rho$ & 6.1 \\
\hline$x_{e}$ & 2.2 .5 & $n_{H}$ & 2.1 .3 & $\rho_{\alpha r}$ & 2.2 .1 \\
\hline$\lambda H_{j}$ & 2.2 .6 & $n_{\mathrm{He}}$ & 2.1 .2 & $S_{\mathrm{bp}}$ & 9.3 \\
\hline$D_{A}$ & 2.2 .2 & $n_{11 j}$ & 2.1 .1 & $S_{\text {fus }}$ & 9.2 \\
\hline$D_{H}$ & 2.2 .3 & $n_{1}$ & 2.1 .3 & $S_{G j}^{\prime}$ & 5.1 .1 \\
\hline$D_{\mathrm{He}}$ & 2.2 .2 & $n_{l j}$ & 2.1 .3 & $S_{H j}$ & 5.1 .2 \\
\hline$D_{H, j}$ & 2.2 .2 & $\dot{n}_{\|}$ & 2.1 .5 & $S_{\mathrm{inj}}$ & 7.1 \\
\hline$D_{w j}$ & 4.1 & $n_{T}$ & 2.1 .6 & $S_{p j}$ & 10.3 \\
\hline$\Delta$ & 6.5 & $n_{0}$ & 5.1 .5 & $\sigma_{s}$ & 5.3 .2 \\
\hline$E$ & 6.5 & $\nu_{\epsilon}$ & 2.2 .1 & $T_{e}$ & $2.1,5$ \\
\hline$E_{Z}$ & 2.1 .7 & $\nu_{H, j}$ & 2.2 .1 & $T_{i}$ & 2.1 .6 \\
\hline$\eta_{f}$ & 6.6 .1 & $\nu_{* i \gamma}$ & 2.2 .1 & $\tau_{e}$ & 2.2 .1 \\
\hline$\eta_{i \|}$ & 2.2 .8 & $p_{b \|}$ & 7.1 & $\tau_{\| H \mathrm{He}}$ & 5.3 .1 \\
\hline$\eta \perp$ & 2.2 .4 & $p_{b \perp}$ & 7.1 & $\tau_{\| H j}$ & 5.3 .1 \\
\hline$\Phi$ & 2.2 .10 & $\dot{p}_{\mathrm{c} x}$ & 5.1 .3 & $\tau_{\| I j}$ & 5.3 .1 \\
\hline$\Phi_{L}$ & 5.3 .2 & $p_{j k}$ & 3.8 & $V_{\rho}^{\prime}$ & 6.3 \\
\hline$\gamma$ & 5.3 .2 & $p_{\phi}$ & 2.1 .4 & $v_{c x}$ & 2.2 .1 \\
\hline$\Gamma_{\mathrm{He}}$ & 2.1 .2 & $\dot{p}_{\phi}^{T}$ & 7.1 & $v_{\phi}$ & 2.1 .4 \\
\hline$\Gamma_{H j}$ & 2.1 .1 & $q$ & 2.2 .1 & $V_{p}$ & 6.3 \\
\hline$\Gamma_{I j}$ & 2.1 .3 & $q_{\mathrm{cx}}$ & 5.1 .4 & $v_{\text {puch }}$ & 2.2 .2 \\
\hline$G_{j}(y)$ & 5.1 .2 & $q_{\mathrm{ec}}$ & 8 & $Z_{\text {eff }}$ & 2.2 .1 \\
\hline$H_{j}$ & 2.2 .3 & $q_{\text {fus }}^{e}$ & 9.1 & $Z_{\text {eff }}^{i}$ & 2.2 .1 \\
\hline$H_{j, k}(\rho)$ & 7.2 .1 & $q_{\text {fus }}^{i}$ & 9.1 & $Z_{11 \mathrm{e}}$ & 2.1 .5 \\
\hline$I(\rho)$ & 2.1 .7 & $q_{i n j}^{e}$ & 7.1 & $Z_{j}$ & 2.1 .3 \\
\hline$t$ & 2.2 .1 & $q_{\text {inj }}^{i}$ & 7.1 & $\begin{array}{l}\left\langle Z_{j}^{2}\right\rangle \\
{[Z]}\end{array}$ & $\begin{array}{l}2.1 .3 \\
201\end{array}$ \\
\hline
\end{tabular}




\section{List of Figures}

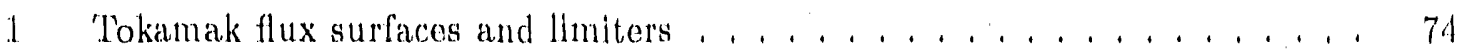

2 Axisymmetric flux surface geometry $\ldots \ldots \ldots \ldots \ldots$

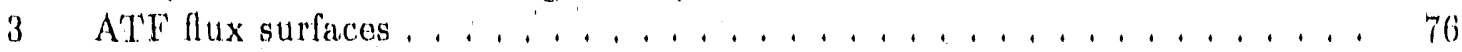

4 Scrape-off layer flux surfaces $\ldots \ldots \ldots \ldots \ldots \ldots \ldots$

5 Equivolume axisymmetric surfaces ................ 78 
ORNL.DWG $80-2729$ FED

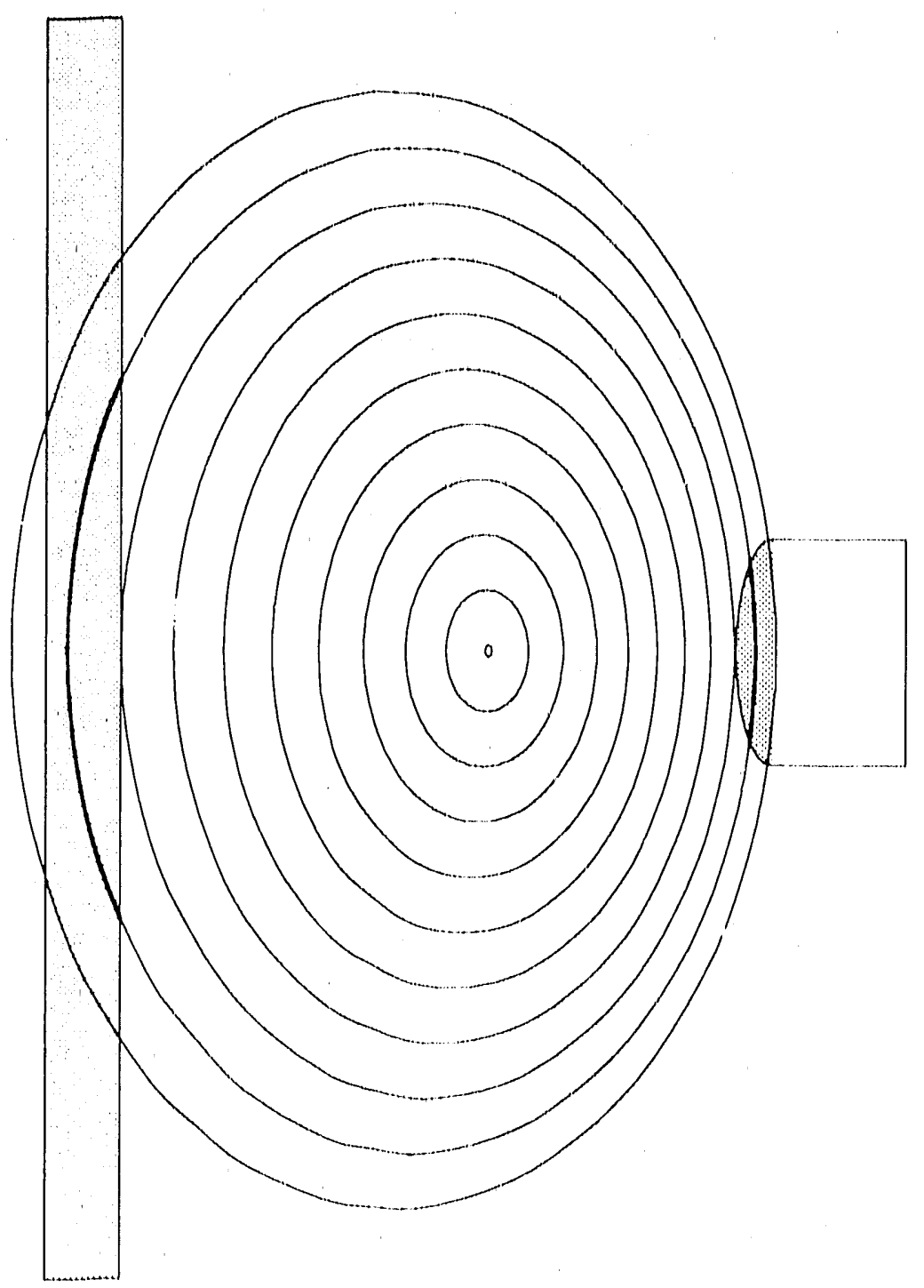

Fig. 1: Intersection of scrape-off flux surface and limiters. For a scrape-off flux surface $\rho$, the arc length $s(\rho)$ used in the definition of the parallel loss time $\tau_{\|}$is the sum of the intersection lengths of the cross section of the flux surface with all limiters. Thus, for the middle scrape-off layer surface shown above, $s(\rho)$ is the sum of the shaded are lengths that intersect the inner rail and outer mushroom limiters. 


\section{ORNL.DWG 90.2730 FED}

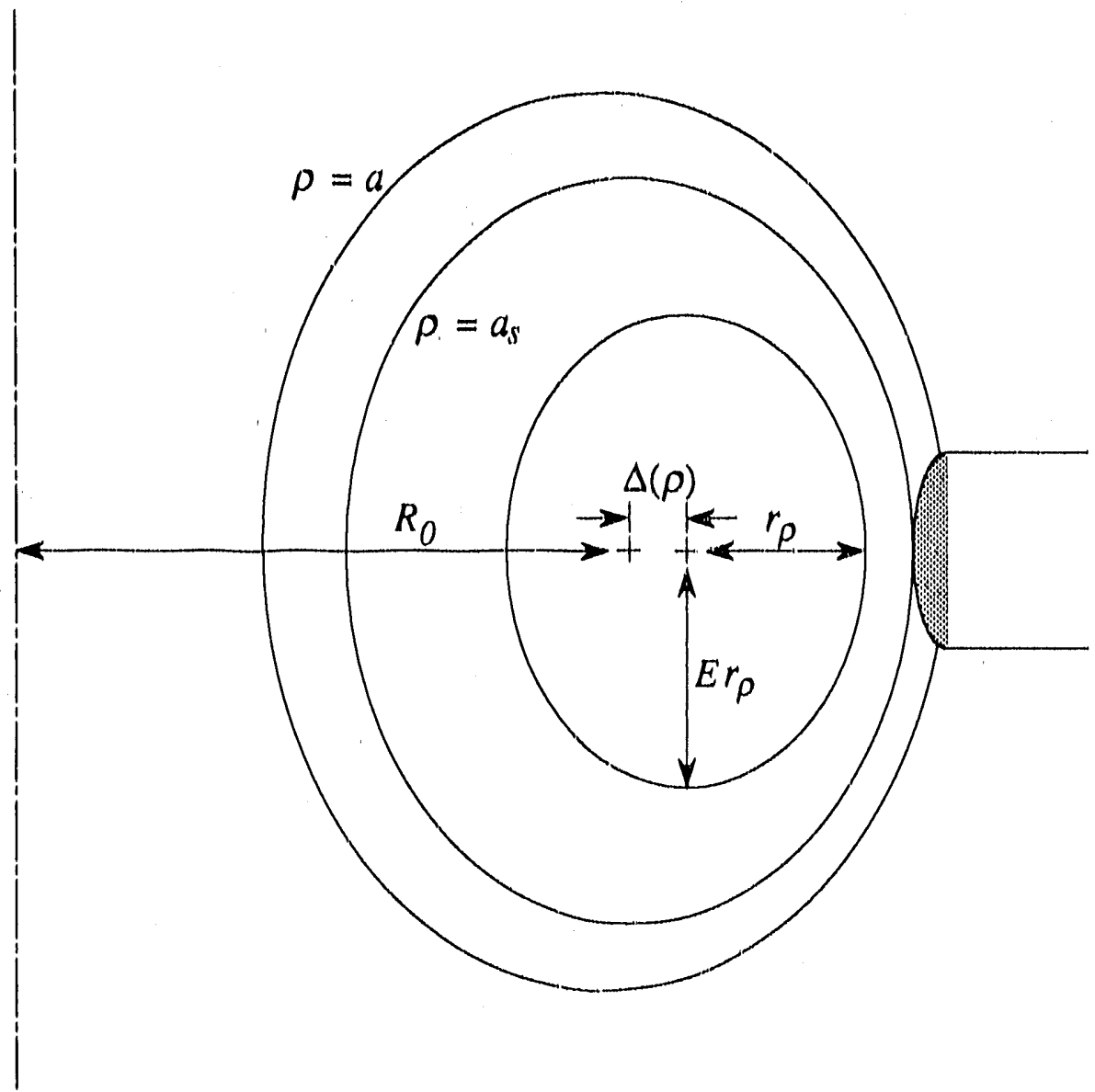

Fig. 2: Axisymmetric flux surface geometry. The cross section of each toroidal flux surface $\rho$ is an ellipse with minor radlus $r_{\rho}\left(=r_{s} \rho\right)$ and elongation $E$ centered at major radius $R_{M}(\rho)=R_{0}+\Delta(\rho)$. The smallesi flux surface that contacts a material surface is labeled $\rho=a_{s}\left(a_{s}=1\right)$, has minor radius $r_{s}$, and is centered at major radius $R_{0}$. Tho shift $\Delta(\rho)$ of surface $\rho$ is with respect to the surface $\rho=a_{s}$; that is, $\Delta\left(\rho=a_{s}\right)=0$. The outermost flux surface on which the plasma solution is obtained is at $\rho=a$. The scrape-off' layer is the region $a_{s}<\rho<a$. 

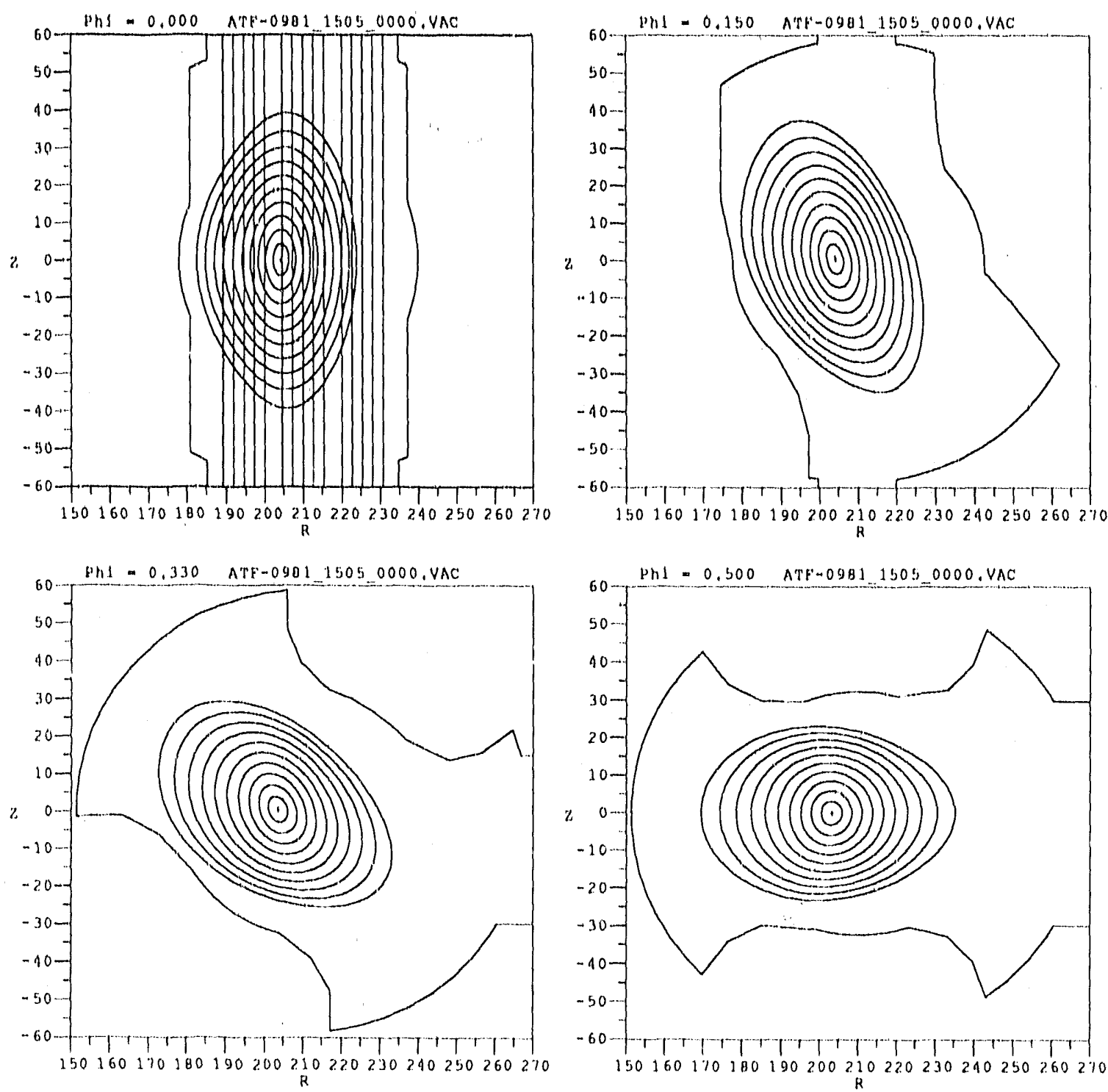

Fig. 3: Vacuum flux surfaces $(6.1 .3)$ calculated for ATF at equal $\rho$ intervals. The toroidal angle of each cut is given at the top of the plot by the fraction of a field period $f_{p}$. The vacuum vessel cross section is also shown. The FIR array chords are shown at $f_{p}=0$. The ratios of the poloidal coil currents to the helical coll current are -0.0981 (outer trim coil), +0.1505 (inner coil), and 0.0 (mid coil). For the outermost surface shown, $t=1.0$. 
ORNL.DWG 90-2732 FED

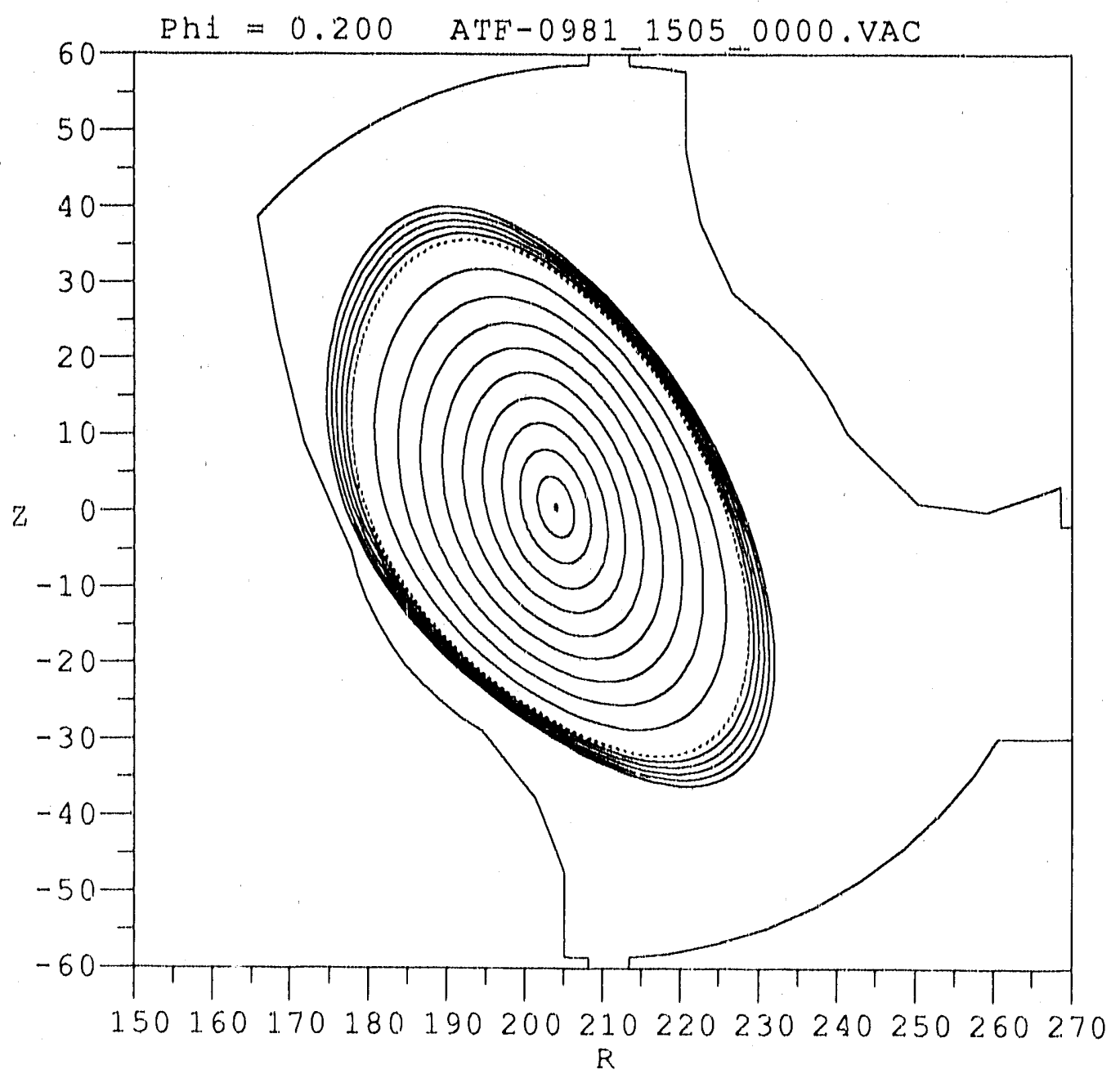

Fig. 4: Vacuum flux surfaces for ATF showing extrapolated scrape-off layer surfaces. The last closed surface $(\rho=1)$ is dashed. Scrapeoff surfaces outside $\rho=1$ are lincar extrapolations of the inner surfaces $(6.1 .5)$. 


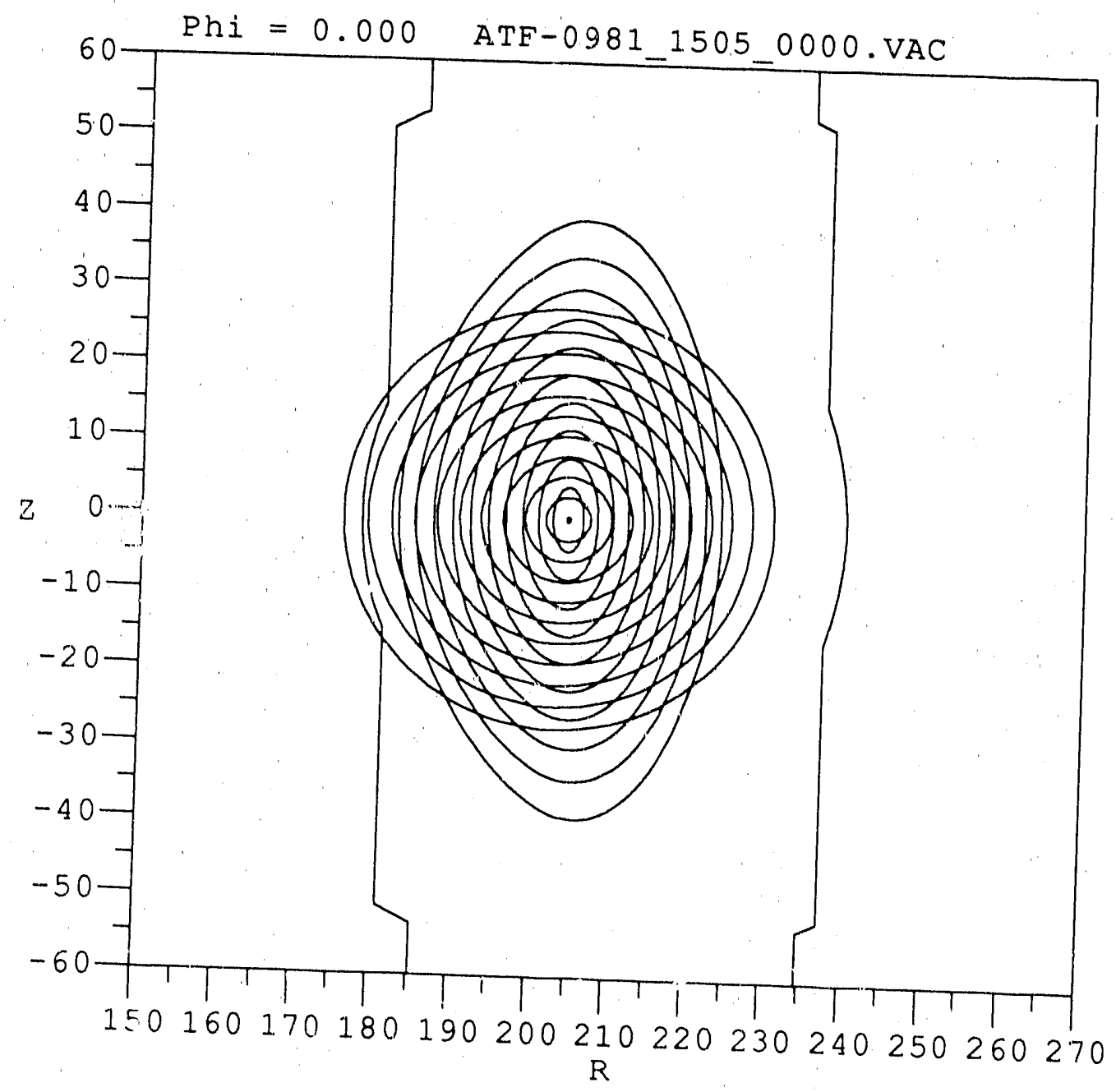
Fig. 5: Superposition of 3-D surfices and corresponding equiyolume axisymmetric
shifted circular surfaces (6.1.6). 

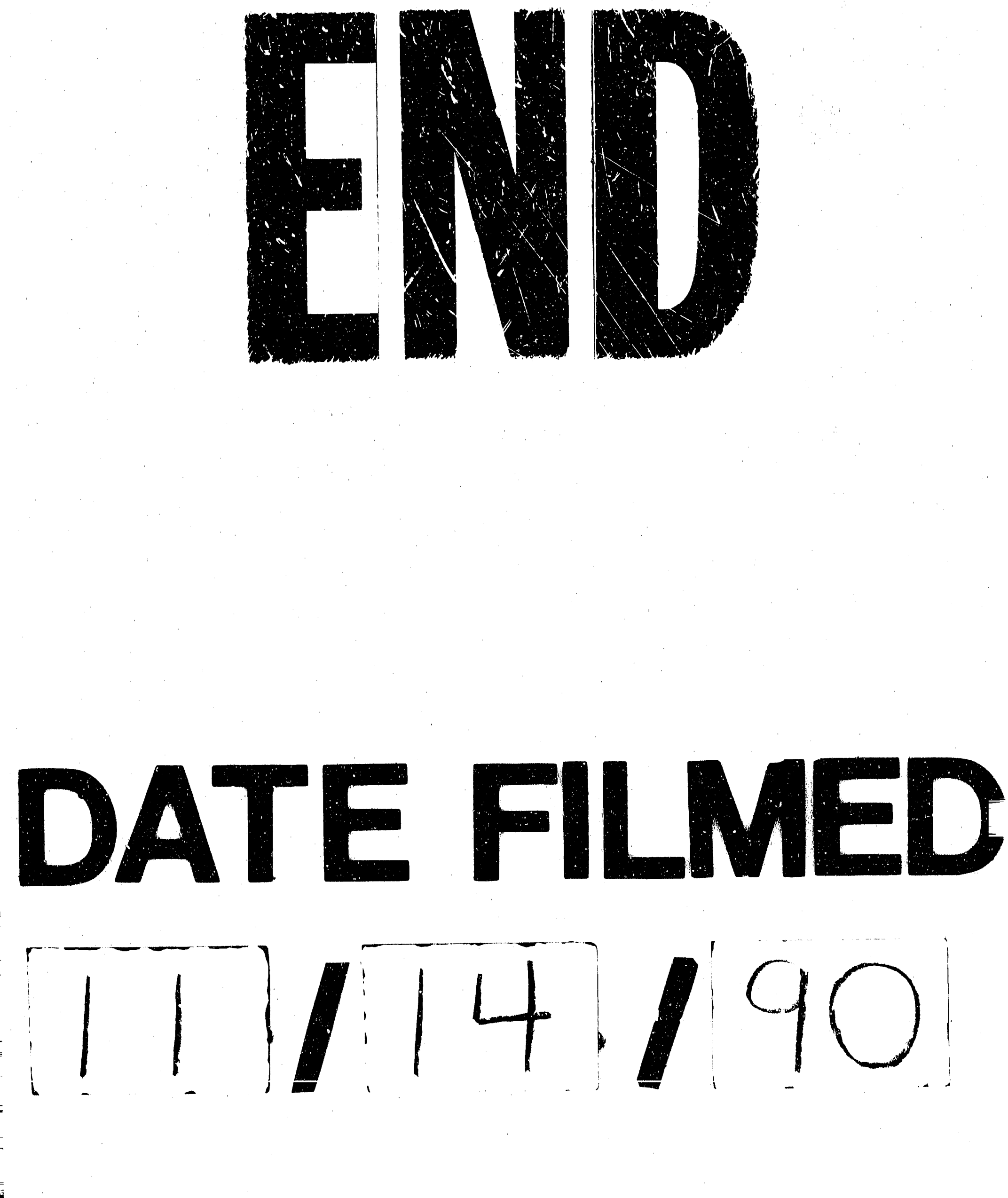
